CARNEGIE INSTITUTE OF TECHNOLOGY

THESIS

EVAPORATION OF METAL CRYSTALS

JOHN PRICE HIRTH 
Page No.

List of Figures ....................... iii.

Iist of Tables..........................

Abstract. .......................... v vii

Acknowledgements...................... viii

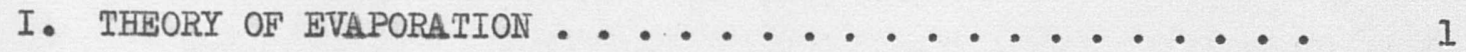

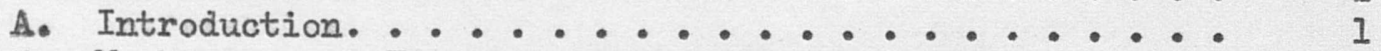

B. Mechanism and Kineties of Crystal Evaporation . . . . 4

1. Preliminary Considerations........... 4

2. The Surface Diffusion Problem ......... 10

3. The Dynamics of Monatomic Ledges. . . . . . . 13

C. Spiral-Dislocation Kinetics ............. 20

1. Preliminary Considerations.......... 20

2. Kinetics for an Unbounded Crystal Containing

One Screw Dislocation............ 24

3. Real Kinetics................. 28

D. Predictions of Theory ............... 32

II. EXPERTMENT. ................... 38

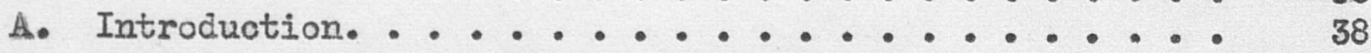

B. Experimental Equipment and Techniques ........ 38

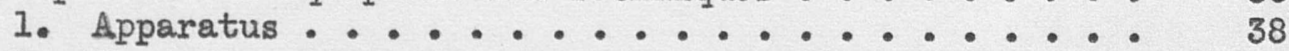

2. Vacurm Technique................ 4 I

3. Specimen Selection and Preparation........ 42

4. Calculation of Results.............. 43

5. Estimated Accuracy of Results ........... 44

C. Bxperimental Results and Discussion......... 47

1. Surface Morphology. . . . . . . . . . . 47

2. Evaporation Rate Measurement. . . . . ... 70

3. Relative Evaporation Rates. . ........ 78

D. Application of Results. . . . . . .... 79

l. Wire Filaments............ 79

2. Catalyst Application. . . . . . ..... 79

3. Langmuir Vapor Pressure Measurements. . . . . 80

4. Other Kinetic Processes ........... 80

III. PROPOSED EXPERTMENTS ................. 81

A. Evaporation Rate Measurements ............ 81

1. Perfect Crystals.............. 81

2. Evaporation Under Varying Undersaturation ..... 81

3. Transient Evaporation Rates .......... 82 
TABLE OF CONTENTS

(Cont' $\mathrm{d}^{\mathrm{s}}$ )

Page No.

B. Surface Morphology ................ 83

1. Perfect Crystals ............... 83

2. Polycrystals ................ 83

IV. SUMMARY. . . . . . . . . ......... 85

V. BIBLIOGRAPHY ................. 89

Appendix I. . . . . . . . . . ........ 94

Appendix II . . . . . . . . . . . . . . . . . . 9 98

Appendix III. . . . . . . . . . . . . . . . . 100

Appendix IV . . . . . . . . . . . .......... 102 


\section{IIST OF FIGURES}

Figure No.

Title

Page No.

1. Schematic view of metal-vapor interface.

2. Surface crossmsection and the distribution of adsorbed atoms on the surface of a crystal.

3. Voriation of $\lambda$ with distance, $x$, from edge. 18

4. Plot of the approximate solution of equation (43). 18

5. A screw dislocation spiral in growth of a crystal. 22

6. A screw dislocation spiral in evaporation of a crystal.

7. Evaporated metal surface at low undersaturation and high temperature.

8. Evaporated metal surface at high undersaturation and low temperature.

9. Evaporated metal surface showing dislocation pitting. 35

10. Schematic diagram of evaporation apparatus.

11. Detailed schematic diagram of specimen holder and furnace.

12. $\{111\}$ surface after evaporation at $623^{\circ} \mathrm{C}$ for 90 min. 49

13. $\{100\}$ surface after evaporation at $623^{\circ} \mathrm{C}$ for 90 min. 49

14. Intermediate position between $\{11\}$ and $\{100\}$ after evaporation at $623^{\circ} \mathrm{C}$ for $270 \mathrm{~min}$.

15. $\{111\}$ surface after evaporation at $623^{\circ} \mathrm{C}$ for 90 min. 50

16. $\{111\}$ surface after evaporation at $623^{\circ} \mathrm{C}$ for varying times.

17. $\{100\}$ surface after evaporation at $623^{\circ} \mathrm{C}$ for $325 \mathrm{~min} .52$

18. Small pits within large pits on polycrystal after evaporation at $847^{\circ} \mathrm{C}$ for $105 \mathrm{~min}$.

19. Intermediate surface showing $\{111\}$ facets after evaporation at $727^{\circ} \mathrm{C}$ for 558 min. 


\section{LIST OF FIGURES}

(cont'd.)

Figure No.

Title

Page No.

20. $\{100\}$ surface after evaporation at $744^{\circ} \mathrm{C}-50 \mathrm{~min}$.

21. $\{100\}$ surface after evaporation at $744^{\circ} \mathrm{C}-770 \mathrm{~min}$.

22. $\{100\}$ surface after evaporation at $744^{\circ} \mathrm{C}-770 \mathrm{~min}$.

23. $\{111\}$ surface after evaporation at $744^{\circ} \mathrm{C}-690 \mathrm{~min}$.

24. $\{111\}$ surface after evaporation at $744^{\circ} \mathrm{C}-690$ min.

25. $\{111\}$ surface after evaporation at $744^{\circ} \mathrm{C}-690$ min.

26. $\{110\}$ surface after evaporation at $644^{\circ} \mathrm{C}$ for 60 min.

27. $\{110\}$ surface after etaporation at $744^{\circ} \mathrm{C}=700 \mathrm{~min}$.

28. $\{110\}$ surface after evaporation at $744^{\circ} \mathrm{C}-700 \mathrm{~min}$.

29. $\{110\}$ surface after evaporation at $744^{\circ} \mathrm{C}-700 \mathrm{~min}$.

62

30. Grain boundary of polycrystal after evaporation at $727^{\circ} \mathrm{C}$ for $528 \mathrm{~min}$.

31. Grain boundary of polycrystal after evaporation at $727^{\circ} \mathrm{C}$ for 528 min.

32. Ledges parallel to grain boundary of chromiun polycrystal.

33. Ledges on chromium polycrystal.

34.

Polycrystal annealed in argon at $800^{\circ} \mathrm{C}$ for two hrs.

64

35. Polycrystal annealed in argon at $800^{\circ} \mathrm{C}$ for two hrs.

67

36. Polycrystal evaporated at $695^{\circ} \mathrm{C}$ for $655 \mathrm{~min}$.

37. Polycrystal evaporated at $744^{\circ} \mathrm{C}$ for 1306 min. 68

38. Evaporation rates of spherical crystals at $727^{\circ} \mathrm{C}$. 71

39. Polycrystal evaporat ed at $623^{\circ} \mathrm{C}$ for one hour. 83

40. Polycrystal evaporated at $623^{\circ} \mathrm{C}$ for one hour. 


\section{LIST OF FIGURES}

(cont ${ }^{2} \mathrm{~d}_{0}$ )

Figure No.

Title

Page No.

Al. Surface profile of ideal crystal.

95

A2. Ledge spacing as a function of distance from the crystal edge.

B1. Laue back reflection of $\{110\}$ pole.

B2. $\{200\}$ reflection using a convergent beam. 


\section{LIST OF TABLES}

Table No.

I. Free energy of activation for position movement.

II. Selected values of $Q$ and $\lambda_{0}$.

III. Fraction of perturbed surface area.

IV. Calculation of $\alpha$ for various crystal planes.

DI. Mean free path of silver in vacuum.
Page No.

8

14

30

74

102 


\section{ABSTRACT}

A mechanism for vaporization of solid metals involving monatomic ledges on metal crystal surfaces is deduced, and an equation is derived for evaporation rate in terms of the dynamies of these ledges. It is shown that crystal edges are a ready source for monatomic ledges. The limiting expression for the vaporization coefficient of large, perfect crystals is given as a function of pressure and temperature by $\alpha=2 / 3\left(p / p_{e}\right)+1 / 3$, where $p_{e}$ is the equilibrium vapor pressure. The effect of screw dislocation-surface intersections on the limiting expression is developed. An equation is derived giving the perturbation due to such intersections. It is found that experimental results for silver crystals confirm various predictions of the theory. Applications of the results are considered. 


\section{ACKNOWLEDGEMENTS}

The author wishes to express his sincere appreciation to Dr. G. M. Pound whose ebullient encouragement and guidance are in a large measure responsible for this work. In addition, the author thanks Drs. F. C. Frank, G. T. Horne, W. Kohn, C. L. McCabe, R. F. Mehl, and L. Yang for helpful discussions; Drs. M. Simnad and J. Chirigos and Messrs. R. G. Hudson and K. L. Moazed for consultation on apparatus design and construction; and $\mathrm{Mr}$. H. Howard for advice on the various mathematical problems encountered on this thesis.

For her patience and encouragement during his years in graduate school, the author expresses his deep gratitude to his wife, Mrs. Martha D. Hirth.

The author thanks Dr. P. G. Shewmon of the Westinghouse Research Laboratories for supplying him with silver single crystals.

Photographic reproductions were made by Mr. F. Volans.

This work was supported by the General Electric Company Fellowship and by the U. S. Office of Naval Research under Contract Nonr $-760(08)$. 
I. THEORY OF EVAPORATION

\section{A. Introduction}

The purpose of this section of the thesis is to denonstrate that an atomistic model of a metal surface and a surface diffusion and deadsorption mechanism can be used in the analysis of evaporation rates of metals to yield results consistent with existing data and to predict certain phenomena to be expected in connection with the vaporization process.*

In the past centennium numerous data relating to rates of evaporation have appeared in the literature. Most of these data have been obtained in the course of vapor pressure experiments, chiefly of the Knudsen (1) and Langmuir(2) types. In the Knudsen experiment, the effusion rate through an orifice in the specimen container is measured to determine the equilibrium vapor pressure. ${ }^{* *}$ The analysis of these data to determine evaporation rates has generally followed the classical treatment due to Hertz $(3)$ and Lengmuir (2) in asserting that the gross rate of vaporization $J$ from a metal surface in equilibrium with its vapor must equal the gross rate of condensation $J_{c}$ on $s u c h$ a surface. Applying the basic kinetic theory of gases, the gross rate of condensation is given by:

$$
J_{c}=\frac{\beta p_{e}}{\sqrt{2 \pi m k T}}
$$

\footnotetext{
* Similar considerations should apply to valence crystals of elements and to simple molecular crystals in the case of monomeric vapors.

** Various geometric coefficients (4-11) which have been discussed in the literature affect the determination of equilibrium vapor pressure from effusion rate measurements. These are not considered in the present work.
} 
where $J_{c}=$ number of atoms per unit area per second, $p_{e}=$ the vapor pressure of the metal at absolute temperature $T, m=$ atomic mass, $k=$ Boltzmann's constent, and $\beta=$ a coefficient equivalent to one minus the fraction of impinging atoms elastically reflected from the surface. Thus, assuming a value for $\beta$, the gross vaporization rate $J=J_{c}$ is dotermined from vapor pressure measurements of the Knudsen type. It is generally accepted that $\beta$ should equal unity for the usual temperatures of vapor pressure measurements. Experimental evidence $(2,12,13)$ indicates that $\beta$ equals unity for clean surfaces of metals with monatomic vapors. A theoretical treatment of this problem for metal surfaces in equilibrium with vapor awaits a treatment analogous to that of Lennard-Jones and Devonshire(14) for the interaction of metal surfaces with gasoous molecules under Van der Wals attractions. In the following development $\beta$ will be taken as unity.

In the Langmuir (2) experiment, the gross rate of vaporization $J$ in a high vacuum is measured directly by weight loss. In order to determine the vapor pressure from these measurements, it is assumed that $J$ is essentially independent of pressure of motal vapor over the surface. Thus, $J$ under a vacuum is assumed to be the same as $J$ under equilibrium vapor pressure $p_{e}$ and hence $p_{e}$ is determined from:

$$
J=\frac{\alpha p_{e}}{\sqrt{2 \pi \text { mkT }}}
$$

where $\mathcal{W}$, the vaporization coefficient is taken as unity. Bxperimental evidence e.g. references (4), (15), (16), (17) indicates that $\propto$ may have values from $10^{-6}$ to 1.0 for the vaporization of metal crystals *

* The vaporization coefficient of liquid metals with clean surfaces is found to be unity. 
with the extremely low values probably due to oxide contamination of the surface. Methods for the experimental determination of $\alpha$ have been reviewed $(18,19)$, and it will be indicated that some are insensitive to the effect of pressure.

An expression for $\alpha$ as a function of pressure and temperature is derived in the following development of an equation for evaporation rate of metal crystals. Only pure metals with clean surfaces and with a monatomic vapor phase will be considered. Further, only unary systems, in which the pressure of metal vapor is the total pressure, are considered. Polanyi and Wigner (20) and Neumann(2I) have presented equations for evaporation rate of crystals. However, the most advanced treatment of metal crystal evaporation is due to Knacke, Stranski, and Wolff(22,23)* , Knacke and Stranski (25). They considered several specific mechanisms involved in vaporization, based on the general mechanism of dissociation from a monatomic ledge, diffusion, and deadsorption. This basic mechanism will be assumed and provisionally justified in the present development. There are several other antecedents which help to justify the assumption of such a mechanism. Burton, Cabrera and Frank(27) have demonstrated that crystal growth from the vapor proceeds by a mechanism of adsorption and surface diffusion to the edge of an advancing monolayer, followed by diffusion along the edge to a kink. Pound, Simnad and Yang (28) showod that heterogeneous nucleation of crystals from metal vapor similarly occurs by a mechanism of adsorption and surface diffusion to an embryo created by statistical fluctuation. In the example of crystal growth, a surface is

* The more complicated case of evaporation of crystalline compounds in which a chemical reaction is involved (e.j. in some axides) has been considered by Knacke, Stranski, and Wolff (22,24) and by J. S. Kane and $L_{\text {. Brewer }}(26)$. 
depicted on which a number of kinks are present in ledges. These ledges at the surface do not, in general, arise from statistical fluctuations but from serew dislocations, stepped monolayers, and other non-equilibrium imperfections.

Experimental evidence for the presence of monatomic ledges has been found in studies of crystal growth $(29,30,31,32)$ and in studies of field. emission tips $(33,34,35)$.

\section{B. Mechanism and Kinetics of Metal Crystal Evaporation}

\section{Preliminary Considerations}

A schematic diagram of a ledge, kink, atoms at a ledge, and adm sorbed atoms on an idealized surface is given in Figure 1. At equilibrium, the state of the surface can be represented by Equations (3) to (7):

$$
\begin{aligned}
& \text { atoms in ledge } \rightleftharpoons \text { atoms in kinks } \\
& \text { atoms in kinks } \rightleftharpoons \text { atoms at ledge } \\
& \text { atoms at ledge } \frac{r_{1}}{r_{2}} \text { adsorbed atoms } \\
& \text { absorbed atoms } \frac{r_{3}}{r_{4}} \text { atoms in vapor } \\
& \text { atoms at ledge } \frac{r_{5}}{\sqrt{r_{6}}} \text { atoms in vapor, }
\end{aligned}
$$

where $r_{1}$ to $r_{6}$ represent the respective rates to be considered in this development. Following Burton, Cabrera, and Frank ${ }^{(27)}$, it is assumed at the outset that the concentration of atoms at a ledge, $n(L)$, in units of

* In general, ledges are not equilibrium structures but are non-equilibrium imperfections in the crystal and raise the chemical potential of the crystal by a slight amount. It is this metastable equilibrium which is being considered. 


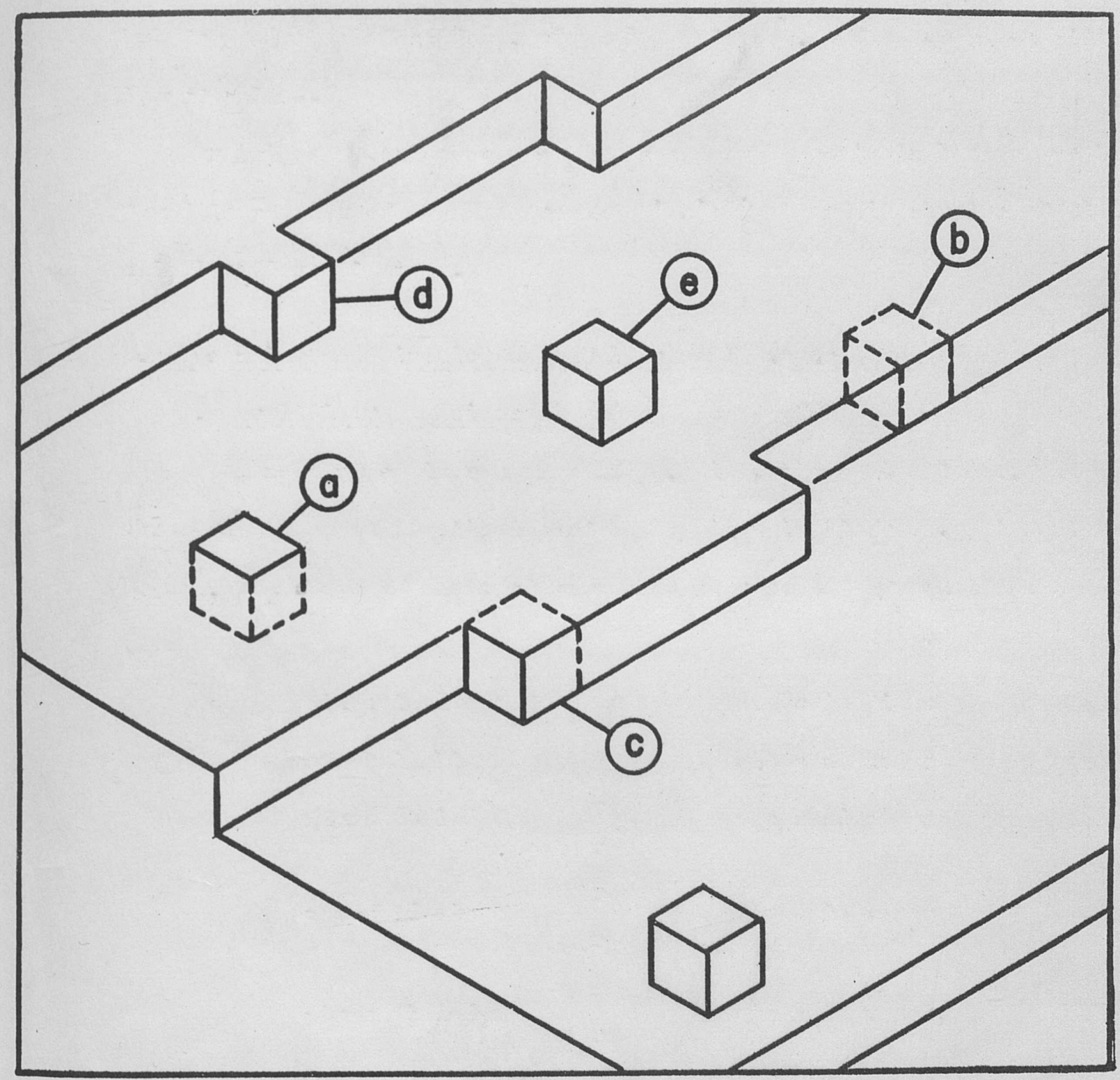

Figure 1

Schematic view of metal-vapor interface depicting ledges, and atoms in the following positions: a, In surface, b. in ledge, c. kink, d. at ledge, and $e$, adsorbed on the surface. 
$\mathrm{cm}^{-1}$, is always the equilibrium concentration $\mathrm{n}(L)_{e}$, even under conditions removed from equilibrium where $p<p_{\theta}$. Provisional justification of this assumption will be given after rates $r_{1}$ to $r_{6}$ have been considered. Further, it is assumed that there are no pressure gradients in the vapor phase. This is a reasonable assumption in view of the rapid diffusion in a vapor at the low pressures of interest in the present unary systems. It is evident that the rates at equilibrium can be written as follows:

$$
\begin{aligned}
& r_{1}=y \cdot n(L)_{e} \nu \exp \left(-\Delta F_{1} / k T\right) \\
& r_{2}=2 y \gamma \cdot n(s)_{e} \nu \exp \left(-\Delta F_{D} / k T\right) \\
& r_{3}=n(s)_{e} \nu \exp \left(-\Delta F_{3} / k T\right) \\
& r_{4}=p_{\theta} / \sqrt{2 \pi m k T} \\
& r_{5}=y^{\circ} n(L)_{e} \nu \exp \left(-\Delta F_{5} / k T\right) \\
& r_{6}=\left(y n_{y} / n_{0}\right) \cdot p_{e} / \sqrt{2 \pi m k T},
\end{aligned}
$$

where the rates (fluxes) are given in units of number of atoms per $\mathrm{cm}^{2}$ of crystal surface per second.

$y=$ the ledge length per $\mathrm{om}^{2}$,

$\nu=$ the vibrational frequency of atoms at the surface, about $10^{13} \mathrm{sec}^{-1}$,

$\gamma=$ probability of a given jump direction in random walk of atoms on the surface, 1/6 for close-packed surfaces, $n(s)_{\theta}=$ the equilibrium concentration of adsorbed atoms, $n_{0}$

$n_{y}=$ the number of atam sites per unit length of ledge, about $3 \times 10^{7} \mathrm{~cm}^{-1}$,

$n_{0}=$ the number of atom sites per unit area of surface, about $10^{15} \mathrm{~cm}^{-2}$, and the $\triangle F^{i} \mathrm{~s}$ are the free energies of activation given in Table $I$ and discussed in the following. $n(I)_{e} \ll n_{y}$ and inasmuch as $\Delta F_{0}$ and $\Delta F_{7}$ 
may be regarded as standard free energy changes:

$$
n\left(I_{\theta}=n_{y} \exp -\left(\Delta F_{0}+\Delta F_{7}\right) / \mathrm{kr} .\right.
$$

The activational enthalpy for each of these reactions can be approximated by considering the net number of bonds broken in the perticular process. Table I is a compilation of the net number of bonds broken for various atom movements on several close-packed surface planes. Only nearest neighbor interactions are assumed for metals with close-packed structures, but both nearest and next-nearest neighbor interactions are considered for metals with body-centered cubic structures. The enthalpy change obtained from the net number of bonds broken is considered a more reasonable approximation to the enthalpy of activation than the total number of bonds broken in processes of atom movement on surfaces, because it is believed that only a fraction of the bond energy $\phi$ is realized as activetional energy in the breaking and remaking of a bond (27). The value of $\varnothing$ listed for surface diffusion is based on Mackenzie's ${ }^{(36)}$ estimate of $\phi / 3$ and experimental indication of $\sim \phi^{(37)}$ for the enthalpy of activation. In the present development, the usual assumption, valid for metals with monatomic vapors, is made that the entropy term may be neglected, and thus $\Delta F$ is equated to $\Delta H$ of activation. The numerical values of the bond energies, and hence of the $\Delta F^{8} \mathrm{~s}$ can be estimated by noting that $\Delta F_{9}$ is approximately the heat of sublimation.

It is now necessary to justify the assumption of a specific mechanism. for crystal vaporization, i.e. to decide whether the vaporization occurs (a) by dissociation from positions at a ledge $\left(r_{1}\right)$ followed by deadsorpm tion $\left(r_{3}\right)$ or $(b)$ by direct evaporation from positions at a ledge $\left(x_{5}\right)$ or by both processes. This question can be decided for equilibrium conditions 
Table I.

Free Energy of Activation for the Change in Position Noted in Relation to

Nearest Neighbor Bonds, $\Phi$, and Next-Nearest Neighbor $\frac{\text { Bonds, } \phi^{\prime}}{\text { To }}$

Kink to At Ledge Adsorbed At Ledge Surfrce Kink in Kink to Vapor at Ledge to Adsorbed to Vapor to Vapor Diffusion Ledge Vapor in Surface

\begin{tabular}{lllllllll} 
Position Change & at Ledge to Adsorbed to Vapor to Vapor Diffusion Ledge Vapor in Surface \\
\hline$\Delta F$ of Activation $\Delta F_{0}$ & $\Delta F_{1}$ & $\Delta F_{3}$ & $\Delta F_{5}$ & $\Delta F_{D}$ & $\Delta F_{7}$ & $\Delta F_{9}$ & $\Delta F_{10}$
\end{tabular}

Lattice Plane

\begin{tabular}{|c|c|c|c|c|c|c|c|c|}
\hline$f \cdot c \cdot c$ & (111) & $\varnothing$ & $2 \varnothing$ & $3 \phi+3 \phi^{\prime}$ & $5 \phi+3 \phi i$ & $<\varnothing$ & $\phi / 2$ & $6 \phi+3 \phi^{\prime} 9 \phi+3 \phi^{\prime}$ \\
\hline f.c.c. & $(100)$ & $\phi^{\prime}$ & $2 \phi+\phi^{\prime}$ & $4 \phi+\phi^{\prime}$ & $6 \phi+2 \phi^{\prime}$ & $<\varnothing$ & $\phi y / 2$ & $6 \phi+3 \phi^{\prime} 8 \phi+5 \phi^{\prime}$ \\
\hline$b \cdot c \cdot c$ & $(100)$ & $\phi$ & $\phi$ & $4 \phi+\phi^{\prime}$ & $4 \phi+2 \phi^{\prime}$ & $<\varnothing$ & $\phi^{\prime} / 2$ & $4 \phi+3 \phi^{\prime} 4 \phi+5 \phi^{\prime}$ \\
\hline$b \cdot c . c$ & (110) & $\phi$ & $2 \varnothing$ & $2 \phi+2 \phi p$ & $4 \phi+2 \phi^{\prime}$ & $<\varnothing$ & $\phi: / 2$ & $4 \phi+3 \phi^{\prime} 6 \phi+4 \phi^{\prime}$ \\
\hline $\begin{array}{l}\text { simple } \\
\text { cubic }\end{array}$ & $(100)$ & $\varnothing$ & $\phi+2 \phi^{\prime}$ & $\phi+4 \phi$ & $2 \phi=6 \phi p$ & $<\varnothing$ & $\phi / 2$ & $3 \phi+6 \phi^{\prime} 5 \phi+8 \phi^{\prime}$ \\
\hline $\begin{array}{l}\text { simple } \\
\text { cubic }\end{array}$ & (110) & $\phi$ & $\varnothing$ & $2 \phi+5 \phi ?$ & $3 \phi-5 \phi^{\circ}$ & $<\varnothing$ & $\phi / 2$ & $3 \phi+6 \phi p 4 \phi+7 \phi^{\prime}$ \\
\hline$h_{\bullet} \cdot c_{\bullet} p_{\bullet}$ & $(0001)$ & $\varnothing$ & $2 \varnothing$ & $3 \phi+3 \phi^{\prime}$ & $5 \phi+3 \phi_{1}$ & $<\varnothing$ & $\phi 2$ & $6 \phi=3 \phi^{\prime} 9 \phi+3 \phi^{\prime}$ \\
\hline
\end{tabular}


by inspection of Equations (8) to (13) and Table $I_{0}$ Since $\Delta F_{5}>\Delta F_{3}$ and $n(s)_{e}>y \cdot n(L)_{e}$ under the usual experimental conditions, $r_{3} \gg r_{5}$. This result can also be obtained by applying the principal of microscopic reversibility ${ }^{(38)}$, which states that, at equilibrium, $r_{1}=r_{2}, r_{3}=r_{4}$, and $r_{5} \equiv r_{6}$. Inasmuch as $n_{0} \gg y n_{y}, r_{4} \gg r_{6}$ and hence $r_{3} \gg r_{5}$. Further, since $\Delta F_{5}>\Delta F_{1}, r_{1} \gg r_{5}{ }^{*}$ Thus, at equilibrium, gross vaporization occurs predominantly by mechanism (a), i.e. dissociation of atoms from positions at ledges followed by deadsorption.

Under conditions removed from equilibrium where $p<p_{e}$ and net vaporization is occurring, it is clear that essentially the same mechanism. must operate because $r_{1}$ is much great er than $r_{5^{\circ}}$ In applying equations (8), (11), (12), and (13) to the non-equilibrium case, it suffices merely to substitute $p$ for $p_{e}$. However, in considering equations (9) and (10) for $r_{2}$ and $r_{3}$, respectively, it is evident that $n(s)$ is no longer equal to $n(s)_{\theta}$ due to surface diffusion awry from the ledge. In fact, one must evaluate $n(s)=f(x)$, where $x$ is the normal distence from the ledge, in order to calculate the vaporization rate. This is done by assuming that $n(s)=n(s)_{e}$ near a ledge and solving the surface diffusion problem. The assumption that $n(s)=n(s)_{e}$ near a ledge is then checked by eveluating the surface diffusion flux $J_{s}$ at $x=0$ and computing $n(s)$ near the ledge from

$$
J_{s}=\left(r_{1}-r_{2}\right) / y
$$

* By analogous arguments, it can be shown that direct evaporation or transfer to the adsorbed state of atoms in kinks, in ledges, or in the surface is negligibly slow. Also, it can be shown that atoms in a ledge are not directly transported to positions at the ledge. 
Returning to the assumption that $n(L)=n(L)_{e}$ always, Burton, Cabrera, and Frank ${ }^{(27)}$ provide some justification for it by pointing out that the free energies of activation for formation of a kink from a ledge $\left(\Delta F_{7}\right)$ and transport of an atom from a kink to a position at a ledge $\left(\Delta F_{0}\right)$ are quite low (Table I). Also, in general, the transport of an atom from a kink to a ledge does not decrease the number of kink positions. Further, the distence apart of kinks in a ledge is small with respect to the net mean distance of random walk in surface diffusion ${ }^{(27)}$. Accordingly, there is no appreciable gredient of $n(L)$ along the ledge, and it may be considered as a line source of atoms for surface diffusion whereupon only diffusion in the direction normal to the ledge need be considered in the case of long, parallel ledges.

\section{The Surface Diffusion Problem}

The net flux $J_{v_{x}}$ into the vapor at any point on the surface is equal to $r_{3}=r_{4}:$

$$
J_{v_{x}}=n(s) \nu \exp \left(-\Delta F_{3} / k T\right)-p / \sqrt{2 \pi m k T},
$$

or fixing the temperature,

$$
J_{v_{x}}=c_{1} n(s)-c_{2} p
$$

If $D_{s}$ is the surface diffusion coefficient, the net flux of atoms awry from a ledge is

$$
J_{s}=-D_{s} \frac{d n(s)}{d x} \text {. }
$$

Applying the continuity condition $-\frac{d J_{S}}{d x}=J_{v_{X}}$.

$$
\frac{d^{2} n(s)}{d x^{2}}=\left(C_{1} / D_{s}\right) n(s)-\left(C_{2} / D_{s}\right) p
$$

As will be evident from the following development, the ledges on a surface undergoing evaporation may be considered as long and parallel. Thus, at steady state conditions, $n(s)$ will be a function of the normal distance $x$ 
from a ledge only, and the distribution of $n(s)$ will be symmetrical with respect to two successive ledges. Therefore, the boundary conditions on equation (19) are $n(s)=n(s)_{e}$ at $x=0, x=\lambda_{0}$, and $d n(s) / d x=0$ at $x=\lambda_{0} / 2$, where the subscript e refers to equilibrium conditions and $\lambda_{0}$ is the distance between two adjacent ledges. The solution to equation (19) is:

$$
n(s)=\left[n(s)_{e}-c_{3} p\right]\left[\frac{\cosh Q\left(x-\lambda_{0} / 2\right)}{\cosh \left(Q \lambda_{0} / 2\right)}\right]+c_{3} p
$$

where $C_{3}=C_{2} / C_{1} \equiv \exp \left(\Delta F_{3} / k T\right) / \nu \sqrt{2 \pi m k T}$ and $Q=\left(C_{1} / D_{s}\right)^{1 / 2}=$

$\left.\left(\nu / D_{s}\right) \exp \left(-\Delta F_{3} / k T\right)\right]^{1 / 2}$ and the concentration gradient of adsorbed atoms is given by

$$
\frac{d n(s)}{d x}=Q\left[n(s)_{\theta}-c_{3} p\right]\left[\frac{\sinh Q\left(x-\lambda_{0} / 2\right)}{\cosh \left(Q \lambda_{0} / 2\right)}\right] .
$$

It is of interest to calculate the average value of $n(s)$

$$
\overline{n(s)}=1 / \lambda_{0} \int_{x=0}^{x=\lambda_{0}} n(s) d x \text {. }
$$

because it may be used to compute the net vaporization rate $J_{\nabla}$ from

$$
J_{v}=c_{1} \overline{n(s)}-c_{2} p
$$

The result of this integration is

$$
\overline{n(s)}=\left(2 / Q \lambda_{0}\right)\left[n(s)_{e}-C_{3} p\right] \tanh \left(Q \lambda_{0} / 2\right)+C_{3} p
$$

in which $\lambda_{0}$ is the only quantity remaining undetermined.

$\lambda_{0}$ can be evaluated using two principles that will be developed in the section on the Dynamics of Monatomic Ledges where it is shown that (a) crystal edges are a ready source for monatomic ledges and (b) for the initial condition of a smooth surface, the ledges will tend to attain a maximum velocity due to the change in surface diffusion gradients accompanying the increase in $\lambda$ with distance from the edge. Now the velocity 
of a monatomic ledge is given by

$$
v=-2 D_{s}\left[\frac{d n(s)}{d x}\right]_{x=0} \cdot \frac{a}{n_{y}} .
$$

where $a$ a the width of one row of atoms, and from equation (21)

$$
\nabla=\frac{2 D_{s} a Q}{n_{y}}\left[n(s)_{e}-C_{3} p\right] \tanh (Q \lambda / 2) .
$$

The rate of change of $\nabla$ with $\lambda$ is

$$
\frac{d v}{d \lambda}=\frac{D_{s} a Q^{2}}{n_{y}}\left[n(s)_{\theta}-C_{3} p\right] \operatorname{sech}^{2}(Q \lambda / 2),
$$

which has small values, $\left\langle 0.01\left(\frac{d v}{d \lambda}\right)_{\max }\right.$, for $Q \lambda / 2>3$.

Therefore, for finite crystal sizes, it is evident that $v$ approaches its maximum steady-state value asymptotically and is approximately equal to $\nabla_{\max }$ at $Q \lambda / 2=3$. Accordingly, the sprcing between ledges which may be attained is of the order of

$$
\lambda_{0} \cong 6 / Q
$$

independent of pressure.

This value for $\lambda_{0}$ may be substituted directly into equation (24) for $\overline{n(s)}$. However, it is more convenient to express the evaporation rate in terms of $\alpha$, the desired vaporization coefficient in equation (2), which equals $r_{3}(p=p) / r_{3}\left(p=p_{e}\right)=\overline{n(s)} / n(s)_{e}$. Thus, substituting equation (28) into (24), dividing by $n(s)_{e}$ and noting (28) that

$$
\begin{aligned}
& c_{3} / n(s)_{e}=\exp \left(\Delta F_{3} / \mathrm{kT}\right) / n(s)_{e} \nu \sqrt{2 \pi m k T}=1 / p_{e} \\
& \alpha=2 / 3\left(p / p_{e}\right)+1 / 3 .
\end{aligned}
$$

Hence, at constant temperature, $\alpha$ increases linearly with pressure from $1 / 3$ at $p=0$ to unity at $p=p_{e}$. At $p=0, \alpha$ is independent of temperature. At finite values of $p, \alpha$ decreases with increase in temperature 
due to the increase in $p_{e}$ but never falls below $1 / 3$. Some values of $Q$ and $\lambda_{0}$ for representative metals are given in Table II. The only data characteristic of the individual metals used in obtaining these values are the crystal structure and the heat of sublimation, which is equated to $\Delta F_{3}$ to obtain values for $\phi$. It is seen that $\lambda_{0}$ decreases with increase in temperature. Of course, $\lambda_{0}$ is independent of pressure. These conclusions apply to the case where the distance between sources of ledges, e.g. crystal edges or grain boumdaries, pores, cracks, screw dislocations, macroscopic ledges, etc., is large relative to $\lambda_{0}$ such that ledges have attained their terminal velocity and spacing $\lambda_{0}$ on most of the crystal surface area. Of course, if the diameter of the evaporating crystal is of the order of $\lambda_{0}$ or less, $\alpha$ will be higher and will tend to approach unity, even if the crystal is perfect, as the crystal diameter is decreased. This case is treated in the following section.

3. The Dynamics of Monatomic Ledges

Having reduced the problem of metal erystal evaporation to one of the dynamics of monatomic ledges on the crystal surface, the next step in this development is to inquire into the origin of this train of parallel ledges which proceed across the surface of a crystal undergoing net vaporization. Considering the steady-state progression of ledges inward from the straight edge of a crystal surface (Figure 2), the time $\tau$ which must elapse before a $1 \mathrm{~cm}$. line of atoms is removed from the edge to form a ledge in steady-state evrporation is given by

$$
\int_{0}^{\tau}-D_{s}\left[\frac{d n(s)}{d x}\right]_{x=0} d t=n_{y} .
$$


Table II

Selected Values for $Q$ and $\lambda_{0}$

for Various Metals, Crystal Planes and Temperatures

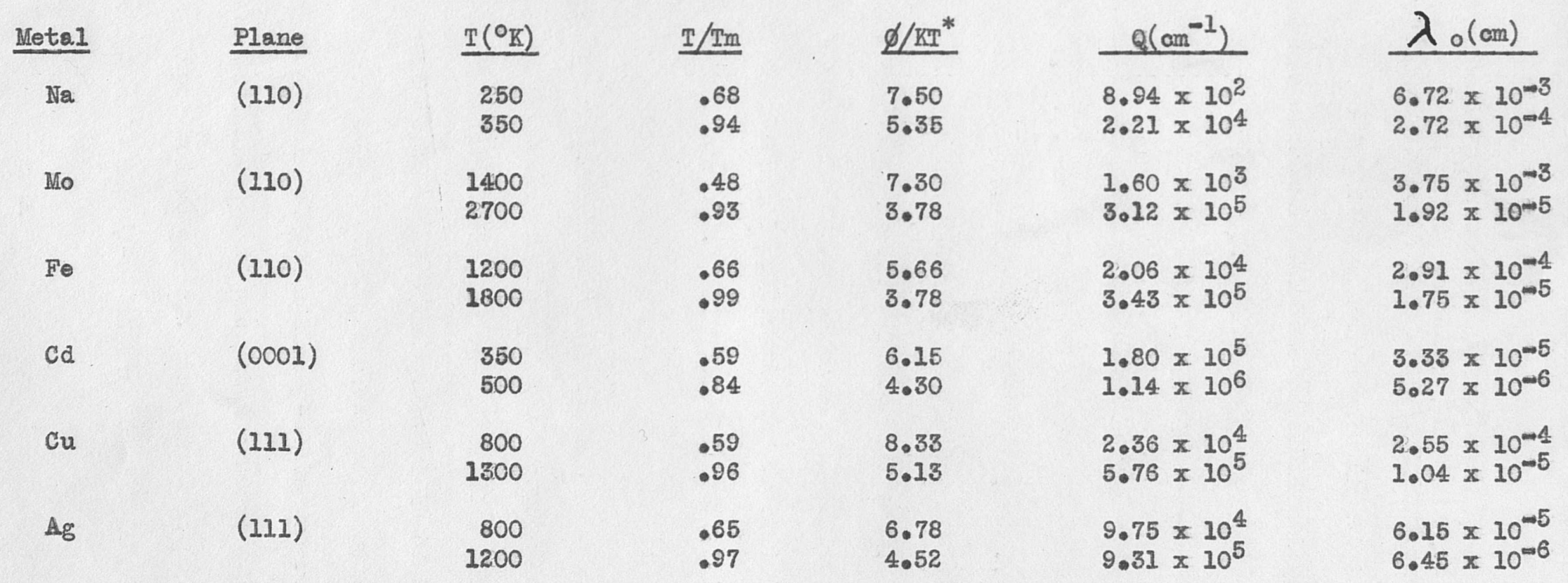

Heats of sublimation were taken from Smithells ${ }^{(39)}$. 
where the gradient refers to the one acting on the edge. Making the reasonable assumption that the velocity $\nabla_{1}$ of the preceding ledge is approximately constant in a small interval of distance X from the edge, $\nabla_{1} \tau=\lambda_{1}$, where $\lambda_{1}$ is the approximate distance between successive ledges in the initial portion of the train. Thus, $d t=d x_{1} / v_{1}$, where $x_{1}$ is the distance from the edge to the preceding ledge, and

$$
\frac{1}{v_{1}} \int_{0}^{\lambda}-\frac{D_{s}}{n_{y}}\left[\frac{d n(s)}{d x}\right]_{x=0} d x_{1} \cong 1
$$

Also, from equation (21),

$$
\left[\frac{d n(s)}{d x}\right]_{x=0}=-Q\left[n(s)_{\theta}-c_{3} p\right] \tanh \left(Q x_{1} / 2\right)
$$

which upon substitution in equation (32) gives

$$
\frac{1}{\nabla_{1}} \int_{0}^{\lambda_{1}} \frac{D_{s} Q\left[n(s)_{e}-c_{3} p\right]}{n_{y}} \tanh \left(Q x_{1} / 2\right) d x_{1}=1
$$

However, from equation (26)

$$
\nabla_{1} \cong \frac{\left.D_{s} \operatorname{aQ}[n(s))_{e}-c_{3} p\right]}{n_{y}} \quad \tanh \left(Q \lambda_{1} / 2\right)
$$

and combining this with equation (34), one gets

or

$$
\begin{aligned}
& \int_{0}^{\lambda_{1}} \tanh \left(Q x_{1} / 2\right) d x_{1} \cong 2 \tanh \left(Q \lambda_{1} / 2\right) \\
& \ln \cosh \left(Q \lambda_{1} / 2\right) \cong \frac{a Q}{2} \tanh \left(Q \lambda_{1} / 2\right)
\end{aligned}
$$

Solving by successive approximation using a typical value of $Q=10^{5}$, $\lambda_{1} \approx$ 10A. Agrin, this value of $\lambda$ is independent of pressure. Thus, the crystal edges serve as a ready source of ledges. 
Next one must demonstrate that the monatomic ledges tend to attain a steady-state maximum velocity as they progress inward from a ledge. Also, it is of interest to determine $\lambda$ as a function of distance $X$ from the edge of a perfect crystal, because then $\overline{n(s)}$ will be known as a function of $X$ and hence the average net flux into the vapor $\overline{J_{\nabla}}$ may be determined from

$$
\overline{J_{v}}=\int_{0}^{X^{\prime}} c_{1} \overline{n(s)} d x-c_{2} p .
$$

where $X^{\prime}$ is half the distance across the perfect crystal surface. Further, a value $X_{0}$ can be obtained which expresses the distance from the edge of the perfect crystal at which $\lambda$ has reached essentially its terminal value $\lambda_{0} \cdot x_{0}$ is the width of the perturbed strip around the periphery of the perfect erystal surface in which $\lambda$ is appreciably less than $\lambda$ 。 and hence $\overline{n(s)}$ and the net evaporation rate are higher. Only perfect crystals considerably larger than $2 x_{0}$ in diameter can have an evaporation coefficient given by equation (30). It may be noted that equation (37) can readily be adapted to the case of imperfect crystals which contain sources of ledges on the surface, e.g. pores, cracks, macroscopic steps, etc., by interpreting $X^{\prime}$ as the half distance between these sources. The rate of increase of $\lambda$ of a ledge $B$ with respect to the time elapsed since it left the edge (Figure 3) in steady-state evaporation is given by

$$
\left(\frac{d \lambda}{d t}\right)_{B}=\nabla_{B}-v_{A}
$$

where the subseript B refers to the ledge just ahead of ledge A. From equation (26), 


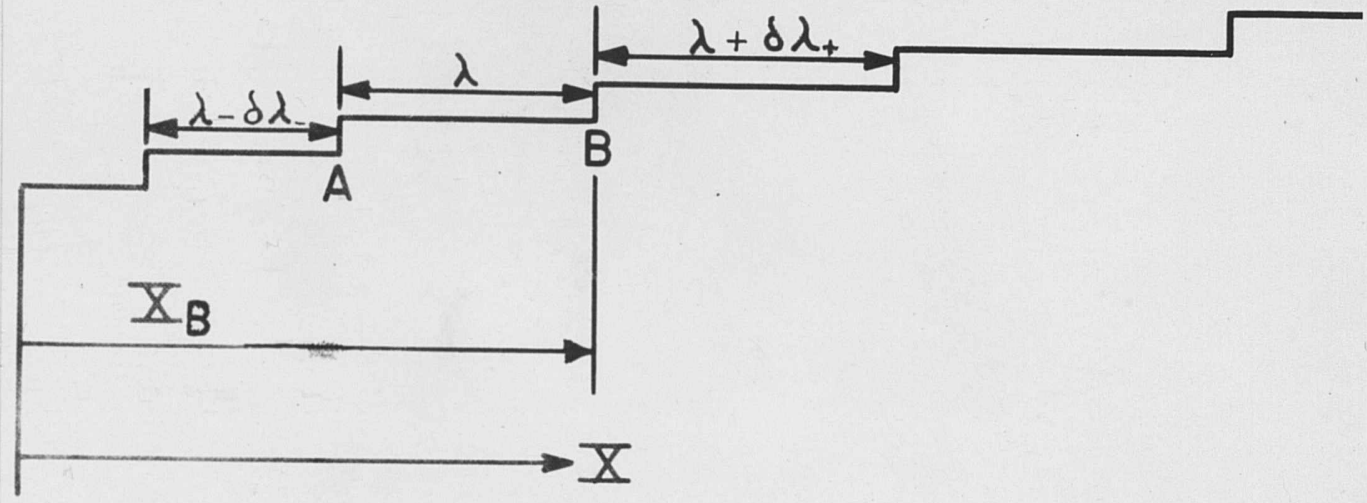

Fig. 3. Variation of $\lambda$ with distance, $x$, from edge.

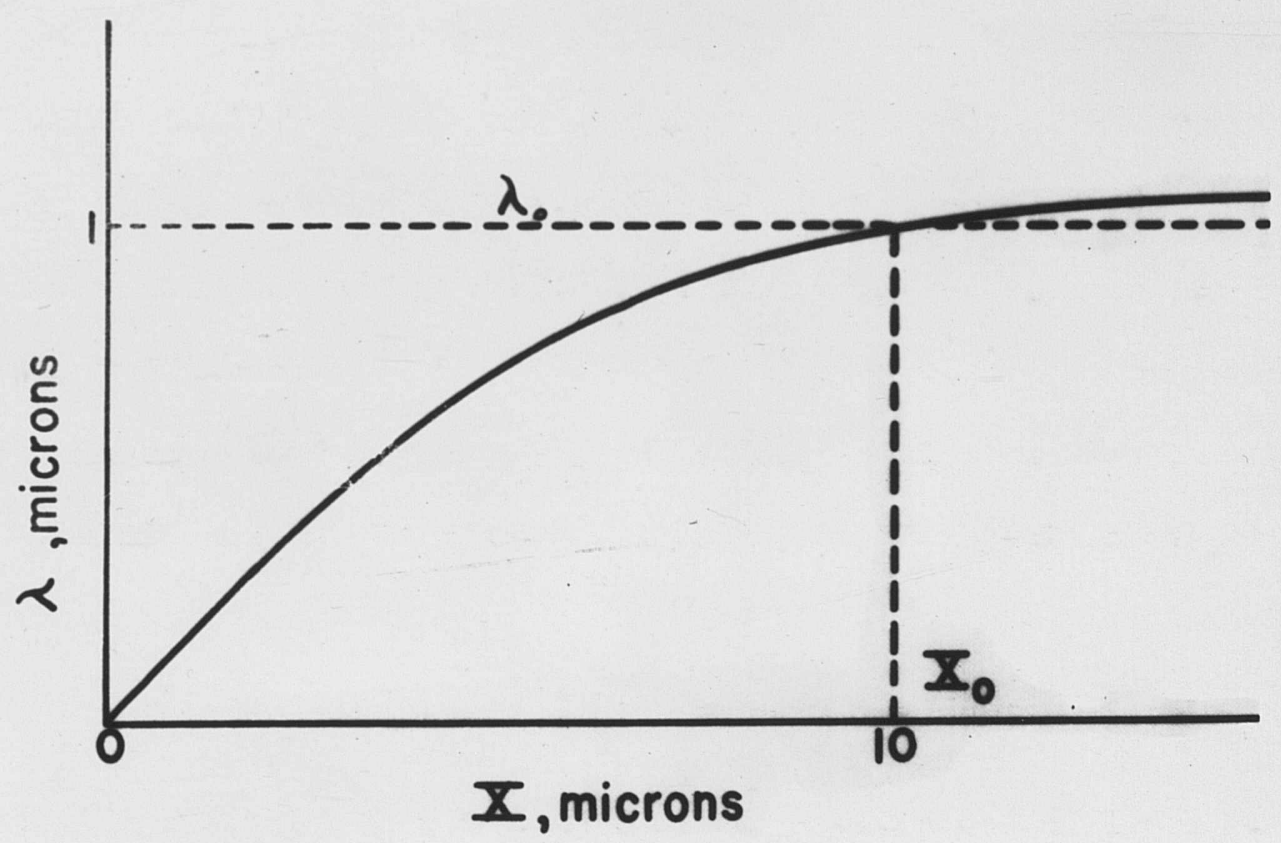

Fig. 4. Plot of the approximate solution of equation (43) for $Q=10^{5}$. 


$$
\begin{aligned}
& \nabla_{B}=\frac{D_{s} Q Q\left[n(s)_{\theta}-C_{3} p\right]}{n_{y}}\left[\tanh \frac{Q}{2}\left(\lambda-\delta \lambda_{+}\right)+\tanh (Q \lambda / 2)\right] \\
& \nabla_{A}=\frac{D_{s Q Q}\left[n(s)_{\theta}-C_{3} p\right]}{n_{y}}\left[\tanh (Q \lambda / 2)+\tanh \frac{Q}{2}\left(\lambda-\delta \lambda_{-}\right)\right] .
\end{aligned}
$$

Also, $d X=v_{B} d t$, and thus

or

$$
\begin{gathered}
\left(\frac{d \lambda}{d x}\right)_{B}=\frac{\nabla_{B}-\nabla_{A}}{\nabla_{B}} \\
\left(\frac{d \lambda}{d X}\right)_{B}=\frac{\left[\tanh \frac{Q}{2}\left(\lambda+\delta \lambda_{+}\right)-\tanh \frac{Q}{2}(\lambda-\delta \lambda-)\right]}{2 \tanh (Q \lambda / 2)} .
\end{gathered}
$$

But

and

$$
\delta \lambda_{+} \approx \lambda\left[\left(\frac{d \lambda}{d \bar{x}}\right)_{B}+\frac{\lambda}{2}\left(\frac{d^{2} \lambda}{d x^{2}}\right)_{B}\right]
$$

$$
\int_{-} \approx \lambda\left[\left(\frac{d \lambda}{d x}\right)_{B}-\frac{\lambda}{2}\left(\frac{d^{2} \lambda}{d x^{2}}\right)_{B}\right]
$$

from which

$$
\begin{aligned}
& \left(\frac{d \lambda}{d x}\right)_{B} \cong\left\{\tanh \frac{Q \lambda}{2}\left[1+\left(\frac{d \lambda}{d x}\right)_{B}+\frac{\lambda}{2}\left(\frac{d^{2} \lambda}{d x^{2}}\right)_{B}\right]\right. \\
& \left.-\tanh \frac{Q \lambda}{2}\left[1-\left(\frac{d \lambda}{d x}\right)_{B}+\frac{\lambda}{2}\left(\frac{d^{2} \lambda}{d x^{2}}\right)_{B}\right]\right\} \\
& 2 \tanh (Q \lambda / 2)
\end{aligned}
$$

Using a typical value of $Q=5.17 \times 10^{5}$, and using a redueed form of equation (43), an iterative calculation of $\lambda(x, t)$ was performed on an electronic computor (see Appendix I). The result was that $\lambda$ approaches the terminal value $\lambda_{0}$ at $x_{0} \cong 10 \lambda_{0}$. An iterative caleulation starting from non-steady state conditions yields the same result as that shown in Figure 4. In view of equations (26) and (27), it is evident that the 
monatomic ledge velocity increases with distance from an edge and essentia.lly attains its maximum velocity at $x_{0} \cong 10 \lambda_{0}$ where $\lambda . \lambda_{0}$. It is seen that, in view of the small values of $\lambda_{0}$ (Table II), positive deviations of $\alpha$ from the value predicted by equation (30) should be observed only in metal crystals of small size (of the order of 1 to 100 microns in diameter, dependent on the metal, temperature, and crystal plane in question). Of course, this conclusion applies only to perfect crystals, i.e. crystals free of pores, cracks, screw dislocations, macroscopic steps, etc.

The problem of evaporation of imperfect crystals, i.e. crystals containing high concentrations of pores, cracks, macroscopic steps, etc., can be solved by the tedious process of integrating over the curves obtained from the computers to obtain solutions for equation (37) in which $\mathrm{X}$ is taken as the half distance between these sources of monatomic ledges.

\section{Spiral-Dislocation Kinetics}

1. Preliminary Considerations

As noted above, imperfections such as grain boundaries, cracks, and pores should act analogously to crystal edges as ledge sources. However, dislocation-surface intersection sites yield a special case of kinetics.

The induction that screw dislocations intersecting a surface are responsible for the growth of crystals from the vapor at very low superm saturations was made by Frank ${ }^{(40)}$ and Burton and Cabrera ${ }^{(41)}$ in 1949. Since that time numerous experimental confirmations, in the form of 
observations of growth spirals emanating from screw dislocation intersections, have been found, as reviewed by Verma (42). The kinetics of spiral growth, and of other growth forms based on the motion of assumed monatomic ledges, was developed by. Burton, Cabrera and Frank ${ }^{(27)}$, and Frank (43).

Cabrera and Levine ${ }^{(44)}$ pointed out that the kinetics of evaporation of spirals emanating from a surface-screw dislocation intersection would be analogous to the treatment of growth mentioned above (27). Typical growth and evaporation spirals are shown in Figs. 5 and 6 . The results of this treatment for the case in which strain energy at the dislocation is neglected indicate that the rate of evaporation can be given by

$$
J=\frac{\nabla d}{\lambda}
$$

where $J$ is the gross flux of evaporation, $d$ is the density of the metal in atoms per $\mathrm{cm}^{2}, \mathrm{v}$ is the velocity of advance of ledges far from the origin, and the spacing between ledges is

$$
\lambda=19 \rho_{\mathrm{c}}
$$

$\rho_{c}$ being the critical radius of curvature of a monatomic disc-shaped nucleus. Thus it is indicated that $\lambda$ will decrease with increasing undersaturation.

It has been shown in the preceding section that evaporation could also take place by the advance of monatomic ledges emanating from crystal edges. In this treatment, although the evaporation rate follows equation (44), $\lambda$ approaches a limiting value $\lambda$ o given by

$$
\lambda_{0}=6 \sqrt{\frac{D_{s} \exp \left(\Delta F_{3} / k T\right)}{\nu}} \text {. }
$$


Figure 5

A Screw Dislocation Spiral in Growth of a Crystal

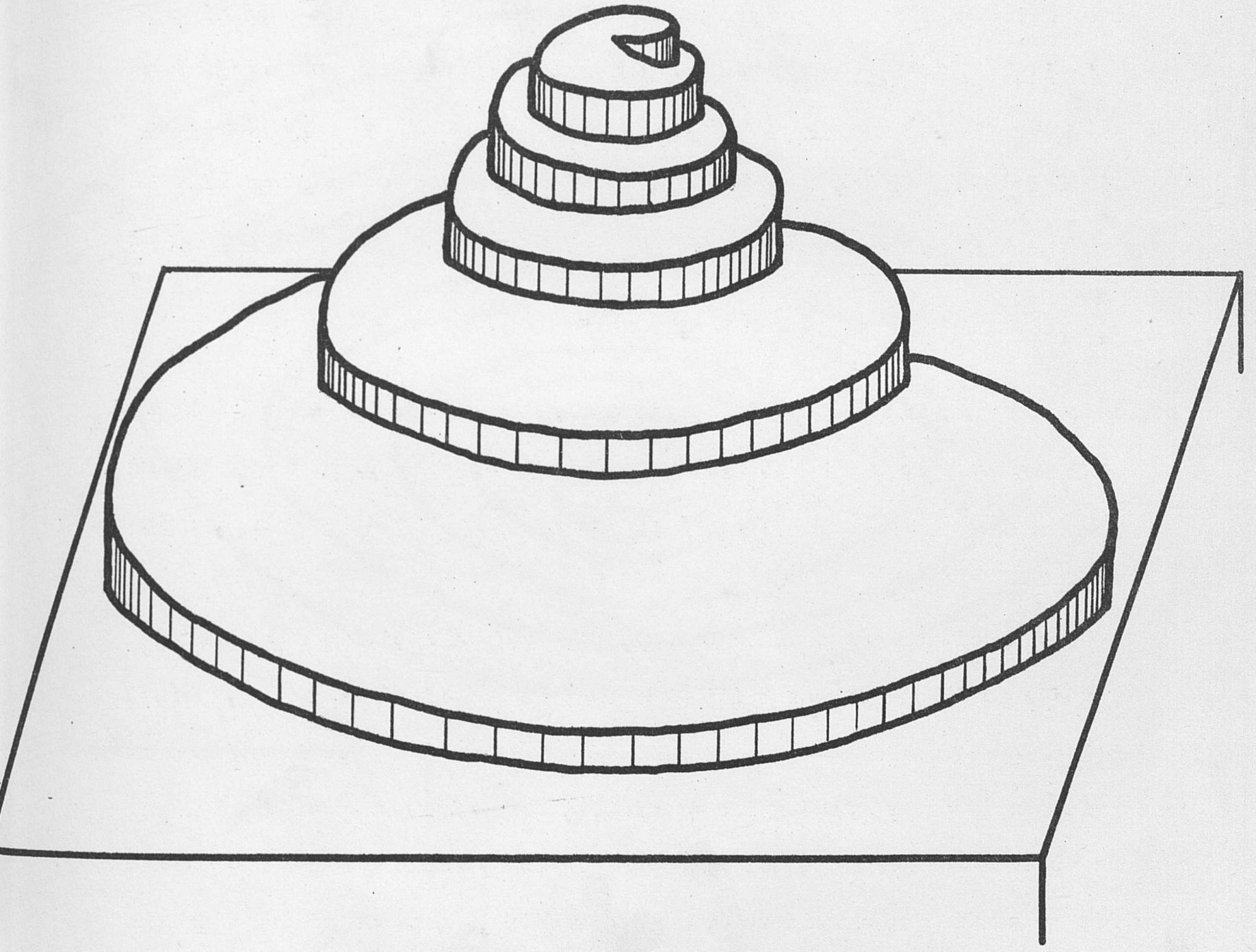


Figure 6

A Screw Dislocation Spiral in

Evaporation of a Crystal

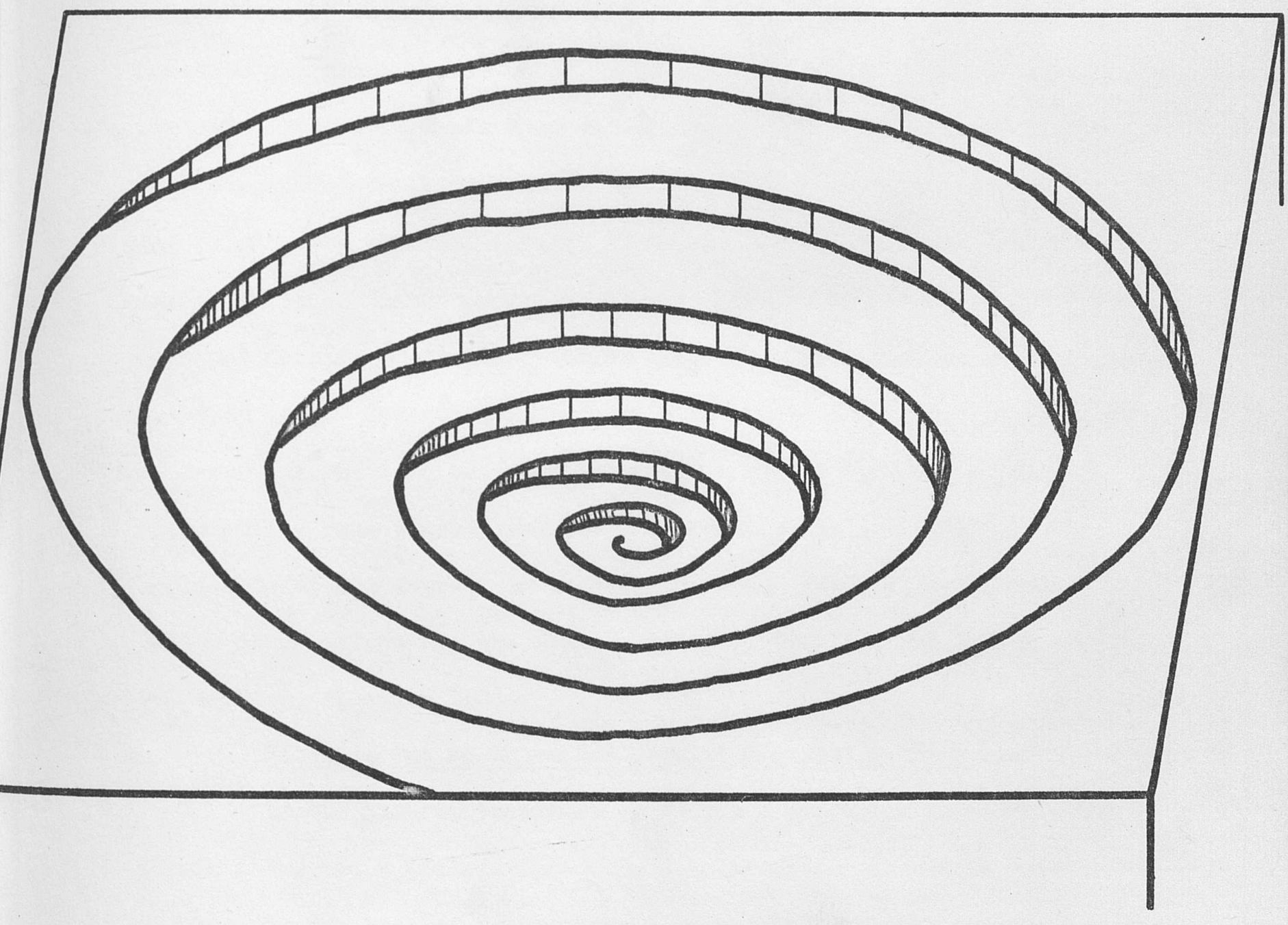


where $D_{s}$ is the surface diffusion coefficient, $\Delta F_{3}$ the free energy of adsorption of an atom onto a surface, and $\mathcal{V}$ the lattice vibrational frequency of the crystal. It is apparent that $\lambda_{0}$ is independent of undersaturation in this treatment.

Considering equations (44), (45) and (46), Frank (45) pointed out that evaporation kinetics should be controlled by the dynamics of screw dislocation spirals at large undersaturations and by the dynamics of edgenucleated ledges at small undersaturations. It is the purpose of the following development to show that, except for a perturbed region near the origin, the terminal spacing of ledges emanating from a dislocation spiral at large undersaturations should approach the value $\lambda$ o for the terminal spacing of straight ledges emanating from odges as given by equation (46). Accordingly, for the case of reasonably low concentration of screw dislocations and high temperature (which produces a small $\lambda_{0}$ ), the limiting case kinetic treatment of straight ledges should be applicable at all degrees of undersaturation. Further, a general criterion is presented for the applicability of the limiting law of crystal evaporation, equation (30).

2. Kinetics for an Unbounded Crystal Containing One Screw Dislocation In the general treatment of spiral evap oration kinetics, the strain energy at a dislocation-surface intersection should be considered ${ }^{(44)}$. However, in the case of interest here, i.e. the evaporation of metals by the recession of steps with a height equal to a Burger's vector of one or two angstrom units, this contribution to the evaporation rate can be neglected. As has been domonstrated, the rate controlling mechanism of evaporation of metals with monatomic vapors is one of diffusion of atoms 
away from monatomic ledges and deadsorption into the vapor phase.

The net evaporation rate from a crystal surface can be given as:

$$
J_{\nabla}=n(s) \nu \exp \left(-\Delta F_{3} / \mathrm{kT}\right)-\mathrm{p} / \sqrt{2 \pi \mathrm{rmkT}},
$$

where $\mathrm{n}(\mathrm{s})$ is the concentration of homogeneously adsorbed atoms, $p$ the vapor pressure over the surface, and $m$ the atomic mass. By a material balance the evaporation rate must also equal the divergence of the diffusion flux away from the advancing ledges:

$$
J_{v}=-\nabla J_{s}=D_{s} \nabla^{2} n(s) \text {. }
$$

Equating (47) and (48) one obtains

$$
\frac{\partial^{2} n(s)}{\partial \rho^{2}}+\frac{1}{\rho} \frac{\partial n(s)}{\partial \rho}-Q^{2} n(s)=c_{1} \text {, }
$$

where $Q^{2}=\frac{\nu \exp (-\Delta F 3 / k T)}{D_{S}}, C_{1}=\frac{-p}{p_{\theta}} \sqrt{2 \pi m k T}$, and $\rho$ is the radius of curvature of the spiral. The solution to equation (49) is a Bessel function of imaginary argument of zero order ${ }^{(46)}$ :

$$
n(s)=c_{2} I_{0}(z)+C_{3} K_{0}(z)+C_{4},
$$

where $I_{0}$ and $K_{0}$ are the Bessel functions of zero order of the first and second kind, $z=Q \rho, C_{2}$ and $C_{3}$ are arbitrary constants, and $C_{4}=p$ $\exp \left(\Delta F_{3} / K T\right) / \nu \sqrt{2 \pi m k T}=n(s)_{e} \frac{p}{p_{e}} \cdot n(s)_{e}$ is the equilibrium concentration of adsorbed atoms, and $p_{\theta}$ is the equilibrium vapor pressure.

In determining the boundary conditions for this case, the GibbsThomson equation for undirectional curvature is applicable:

$$
\Delta F_{c}=-\frac{\gamma}{\rho}
$$

where $\gamma$ is the specific interfacial free energy, and $\Delta F_{0}$ is the change in free energy of a ledge due to $\rho$, the negative of the radius of curvature. Noting that 


$$
\Delta F_{c}=\frac{k T}{V} \quad \ln \left[\frac{n(s)}{n(s)_{\theta}}\right] \text {. }
$$

where $V$ is the atomic volume, and equating (51) and (52), one obtains

$$
n(s)=n(s) \text { exp }\left(\frac{-\gamma v}{\rho k T}\right) \text {. }
$$

Since the ledge spacing of interest here is the spacing at large $r$, the approximation $\rho \equiv x$ can be inserted into equations (50) and (53). (53) becomes

$$
n(s)=n(s)_{\theta} \quad \exp \left(\frac{-\gamma v}{r k T}\right)
$$

Thus the boundary conditions on (50) are

$$
\begin{aligned}
& n(s)=n(s)_{e} \exp \left(\frac{-\gamma v}{r_{1} k T}\right) \text { at } z=0 r_{1} . \\
& n(s)=n(s)_{e} \exp \left(\frac{-\gamma v}{r_{2} k T}\right) \text { at } z=0 r_{2} .
\end{aligned}
$$

Inserting (55) and (56) in (50), one obtains

$$
\begin{aligned}
& \begin{aligned}
c_{2} & =\left\{\left[n(s)_{e} \exp \left(\frac{-\gamma v}{r_{2} k T}\right)-n(s)_{e}\left(\frac{p}{p_{e}}\right)\right] K_{0}\left(Q r_{1}\right)\right. \\
& \left.-\left[n(s)_{e} \exp \left(\frac{-\gamma v}{r_{1} k T}\right)-n(s)_{e}\left(\frac{p}{p_{\theta}}\right)\right] K_{0}\left(Q r_{2}\right)\right\}
\end{aligned} \\
& c_{3}=\left\{\left[n(s)_{e} \exp \left(\frac{-\gamma_{V}}{r_{1} k T}\right)-n(s)_{e}\left(\frac{p}{p_{e}}\right)\right] I_{0}\left(\theta r_{2}\right)\right. \\
& \frac{\left.-\left[n(s)_{e} \exp \left(\frac{-\gamma_{v}}{r_{2}{ }^{k T}}\right)-n(s)_{e}\left(\frac{p}{p_{e}}\right)\right] I_{0}\left(Q r_{1}\right)\right\}}{K_{0}\left(Q r_{1}\right) I_{0}\left(Q r_{2}\right)-K_{0}\left(Q r_{2}\right) I_{0}\left(Q r_{1}\right)} \text {. }
\end{aligned}
$$

However, the contribution to the ledge free energy due to the curvature can be neglected when

$$
r_{a} \geq \frac{\gamma \nabla}{0.01 \quad k T}
$$

Employing this condition, (50) becomes 


$$
\begin{aligned}
& n(s)=n(s)_{e}\left(1-\frac{p}{P_{\theta}}\right)\left\{\frac{\left[K_{0}\left(Q r_{1}\right)-K_{0}\left(Q x_{2}\right)\right] I_{0}(z)+\left[I_{0}\left(Q x_{2}\right)-I_{0}\left(Q x_{1}\right)\right] K_{0}(z)}{K_{0}\left(Q x_{1}\right) I_{0}\left(Q x_{2}\right)-K_{0}\left(Q x_{2}\right) I_{0}\left(Q x_{1}\right)}\right\} \\
& +n(s)_{e}\left(\frac{p}{p_{e}}\right) \text {. }
\end{aligned}
$$

As noted in equation (44) the relation between $n(s)$ and $v$ is of interest:

$$
v=\frac{-2 D_{s} a}{n(y)}\left[\frac{d n(s)}{d x}\right]_{r=r_{1}} \text {. }
$$

where $a$ is the ledge height and $n(y)$ is the number of atoms per unit length of ledge.

From (60)

$$
\frac{d n(s)}{d r}=\operatorname{Qn}(s)_{e}\left(I-\frac{p}{P_{0}}\right)\left\{\frac{\left[K_{0}\left(Q r_{1}\right)-K_{0}\left(Q r_{2}\right)\right] \frac{d I_{0}(z)}{d z}+\left[I_{0}\left(Q r_{2}\right)-I_{0}\left(Q x_{1}\right)\right] \frac{d K_{0}(z)}{d z}}{K_{0}\left(Q r_{1}\right) I_{0}\left(Q x_{2}\right)-K_{0}\left(Q x_{2}\right) I_{0}\left(Q r_{1}\right)}\right\}
$$

Invoking the formulae $(46)$

$$
\frac{d I_{0}(z)}{d z}=I_{1}(z), \quad \frac{d I_{0}(z)}{d z}=-K_{1}(z)
$$

(61) reduces to

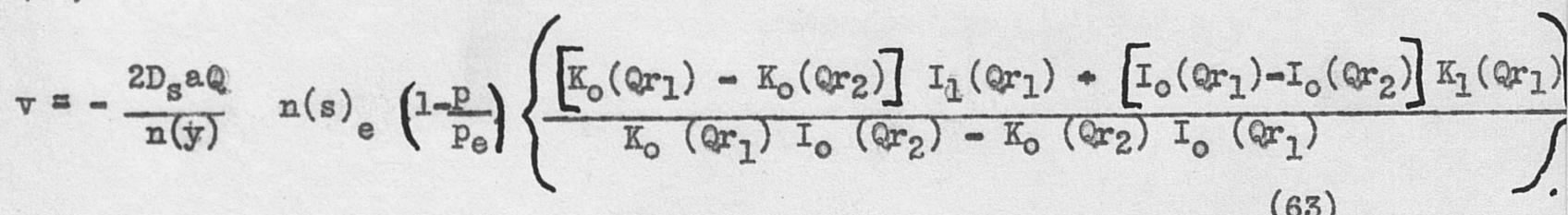

Using the asymptotic expansions for the Bessel functions in (63), one obtains: $\nabla=\frac{2 D_{s} a Q}{n(y)} n(s)_{e}\left(1-\frac{p}{p_{e}}\right)\left[\operatorname{coth} Q\left(r_{2}-r_{1}\right)-\sqrt[2]{\frac{r_{2}}{r_{1}}} \operatorname{csch} Q\left(r_{2}-r_{1}\right)\right]$.

As the argument of the hyperbolic functions approaches 3, the velocity approaches its steady state value asymptotically:

$$
v=\frac{2 D_{s}{ }^{2 Q}}{n(y)} n(s)_{e}\left(1-\frac{p}{P_{\theta}}\right) .
$$


At this condition $r_{2}-r_{1}=\lambda=\lambda_{0} \quad c_{\cdot} f_{\bullet}(28)$ independent of undersaturation as was noted above.

Actually, that $\Delta r=\lambda$ approached $\lambda_{0}$ at large $r$ could have been predicted by the similarity between two adjacent spiral ledges at large $r$ and two parallel straight ledges at large distance from the origin of ledges. However, it is thought that inclusion of the above treatment for circular geometry is desirable because, in conjunction with the relationship c.f•(4I)

$$
\frac{d \Delta r}{d r}=1-\frac{\nabla_{1}}{v_{2}}
$$

equation (64) may be used to evaluate $\Delta r=\lambda$ as a function of $r$. This more complete solution of the problem of spiral evaporation is not included in the present paper because of the difficulty of the iterative computation leading to numerical answers. It should be pointed out that it is possible, at least in principle, to further refine the derivation of equation (64) so that it would be applicable at very small values of $r$ by including the correction for capillarity and by expressing $\rho=$ $\rho(r, \theta)$

\section{Real Kinetics}

It is now apparent that steady state evaporation is described by equations (44) and (46) which indicate that the gross evaporation rate varies linearly from the value $J_{\nabla_{e}}$ at equilibrium pressure to $1 / 3 J_{v_{\theta}}$ at zero pressure. That is, the vaporization coefficient $\alpha$ varies from 1 to $1 / 3$ as the pressure over the surface varies from the equilibrium value to zero. However, perturbations arise near the centers of evaporation spirals which tend to decrease the ledge spacing and increase the 
evaporation rate. It is of interest to estimate the magnitude of these perturbations.

When the spacing of ledges varies from some value, $\lambda$, near the center of the spiral to a greater value $\lambda_{0}$ away from the spiral, as it does in cases when $\lambda_{0}>19 \rho_{c}$ it follows from the above that a true steady state evaporation does not obtain over the entire surface. Rather, the evaporation rate is higher in the region of the spiral center, giving rise to cavities near these centers. In actuality, surface forces or strains arising from the presence of the dislocation may control the limiting acuteness of such cavities. Although no steady state solution may be obtained, one can estimate the perturbed area. around a dislocation in the following way.

First it is of interest to compute the radius of the area affected by capillarity around a dislocation from equation (59). Values of the radius $r_{a}$ of this area affected by capillarity in various metals are given in Table III. These values are appreciable, being generally of the order of $\lambda_{0^{*}}$ Accordingly, one would expect appreciable acceleration of the ledges in this region and accompanying increase in the ledge spacing $\Delta r=\lambda$. This is particularly true in view of the fact that high curvature should enhance ledge acceleration due to both capillary and purely geometric considerations. As explained in section I-C-3, exact evaluation of $\Delta x=\lambda$ as a function of $r$ has not been accomplished. However, by analogy with the treatment for edge nucleated ledges, it is estimated that $\lambda_{a}$ is of the order of $0.5 \lambda_{0}$ at $r_{a}$.

The total area affected by capillarity on a given surface will be a function of $r_{2}$ and of the concentration of screw dislocations intersecting 
Table III

Fraction of Area Affected by Capillarity and Total Fraction of Perturbed Area, Based on a Screw $\frac{\text { Dislocation }}{\text { Concentration of } 105} \frac{1}{\text { per }} \mathrm{cm}^{2}$

\begin{tabular}{|c|c|c|}
\hline zal & ToK & $\left.1 \mathrm{~cm}^{2}\right)$ \\
\hline \multirow[t]{2}{*}{$\mathrm{Na}$} & 250 & $300^{(49,50)}$ \\
\hline & 350 & \\
\hline \multirow[t]{2}{*}{ Mo } & 1400 & $2530^{(49,50)}$ \\
\hline & 2700 & \\
\hline \multirow[t]{2}{*}{$\mathrm{F} \theta$} & 1200 & $2040(49,50)$ \\
\hline & 1800 & \\
\hline \multirow[t]{2}{*}{$\mathrm{Cd}$} & 350 & $410^{(49,50)}$ \\
\hline & 500 & \\
\hline \multirow[t]{2}{*}{$\mathrm{Cu}$} & 800 & $1650^{(51)}$ \\
\hline & 1300 & \\
\hline \multirow[t]{2}{*}{$\mathrm{Ag}$} & 800 & $1130^{(51)}$ \\
\hline & 1200 & \\
\hline
\end{tabular}

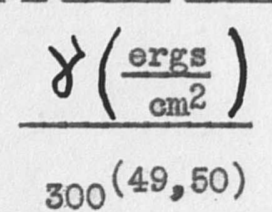

$r_{a}\left(\times 10^{5} \mathrm{~cm}\right)$
3.44
2.44
2.05
1.06
1.45
0.97
1.81
1.27
1.76
1.08
1.75
1.16

$\frac{A_{a}\left(x 10^{4}\right)}{3.46}$
1.92
1.31
0.35
0.65
0.30
1.04
0.51
0.97
0.37
0.96
0.43

. .0
1
.58
1
.002
.62
.002
.009
.0002
.51
.0008
.03
.0003


the surface in question. This dislocation concentration will depend on the prior history of the metal, and will not be thermodynamically stable. However, a reasonable estimate of this concentration in well annealed crystals is taken as $10^{5}$ per $\mathrm{cm}^{2}[$ c.f. $(47),(48)]$. Thus the fraction of the surface affected by capillarity will be

$$
A_{a}=\pi r_{a}{ }^{2} 10^{5} \text {. }
$$

This function is evaluated for representative metals in Table III.

The total perturbed area will include $A_{a}$ and the region where increases from $\lambda_{a}$ at $r_{a}$ to $\lambda_{0}$ at $r_{0}$. Again, the distance $\Delta R$ over which this takes place can be estimated by analogy with the treatment for edge nucleated ledges. There the solution based on an expression similar to equation (66) leads to a total perturbed distance equal to about $10 \lambda_{0^{\circ}}$ Since in this case the spacing must vary from $\lambda_{a} \cong \lambda_{0} / 2$ to $\lambda_{0}$ rather than from 0 to $\lambda_{0}, \Delta R$ is estimated to be $\cong 5 \lambda_{0}$. As explained above, exact evaluation of $\Delta R$ has not been accomplished. However, it is believed that the present approximation yields results correct to an order of magnitude in terms of affected area. Thus the total perturbed fraction will be

$$
A_{0}=\pi\left(x_{2}+5 \lambda_{0}\right)^{2} 10^{5}
$$

These values are given in Table III where values for $\lambda$ o are taken from Table II.

$\left(1-A_{0}\right)$ now represents the fraction of the surface obeying the $\alpha=1 / 3+2 / 3\left(p / p_{e}\right)$ law, and $A_{0}$ represents the fraction wherein $\alpha=$ $A+B\left(p / p_{e}\right), A(\lambda)$ varying from $1 / 3$ to $I$, and $B(\lambda)$ varying from $2 / 3$ to zero. If the spacing near the dislocation center is of the order 
of $\lambda_{0}$ evaporation from almost the entire surface will be described by $A=1 / 3, B=2 / 3$. This occasion arises when the spacing at small $r$ is $\cong 19 \rho_{c}>\lambda_{0}{ }^{(44)}$. Then the specing of ledges emanating from dislocations will be greater than the spacing of ledges emanating from crystal edges, and the dynamics of the latter will govern the evaporation. Hypothetical surfaces showing conditions increasingly favoring dislocam tion pitting are shown in Figs, 7,8 , and 9.

\section{Predictions of Theory}

1. The model of a crystal surface in which monatomic ledges are present and the mechanism of evaporation deduced therefrom lead to rate equations that are consistent with experimental observations. The mechanism involves dissociation of atoms from the monatomic ledges, surface diffusion, and deadsorption. The key to this treatment lies in the dynamics of the monatomic ledges.

2. The limiting expression for the evaporation coefficient defined in equation (2) is $\alpha=2 / 3\left(p / p_{e}\right)+1 / 3$. This should apply to the evaporation of large metal crystals that are relatively free of surface imperfections.

3. Positive deviations from the limiting expression would be expected for erystal (or grain) sizes of the order of 1 to 100 microns or less. This deviation is not due to the Gibbs Thomson effect.

4. Crystal edges should serve as a ready source for monatomic ledges during evaporation. 
Evaporation from a Metal Surface Containing a High or Low Concentration of Screw. Dislocations at Low Undersaturation (high $\mathrm{p}$ ) and $\mathrm{High}$

Temperature Giving No Pitting at Screw

Dislocations and Control of the Evaporation by Ledges Emanating from the Crystal Edges

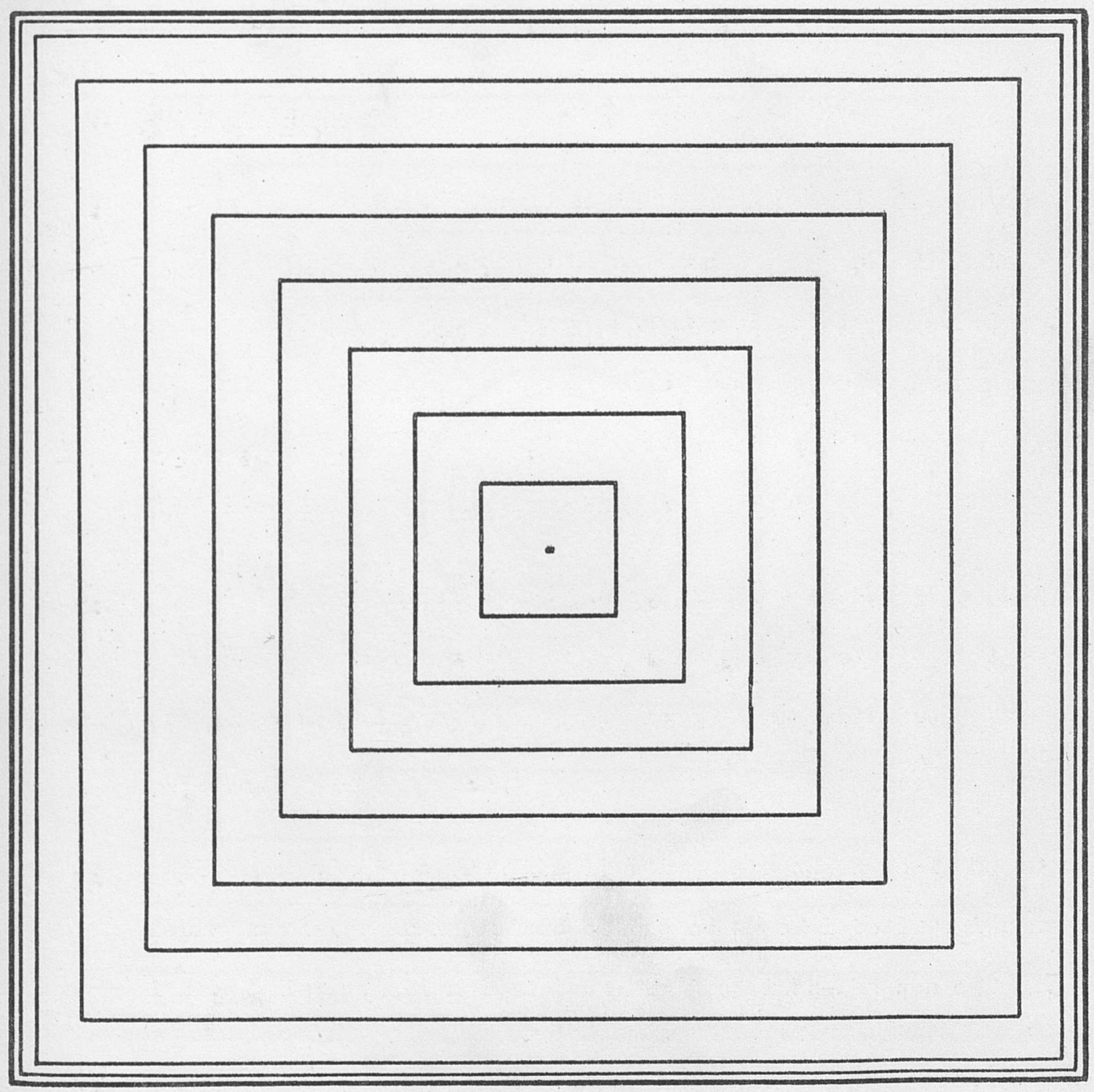


Evaporation from a Metal Surface Containing a Low Concentration of Screw Dislocations at High Undersaturation (low p) and Low Temperature Giving Pitting at Screw Dislocations but Control of the Evaporation by Ledges Emanating from the Crystal Edges

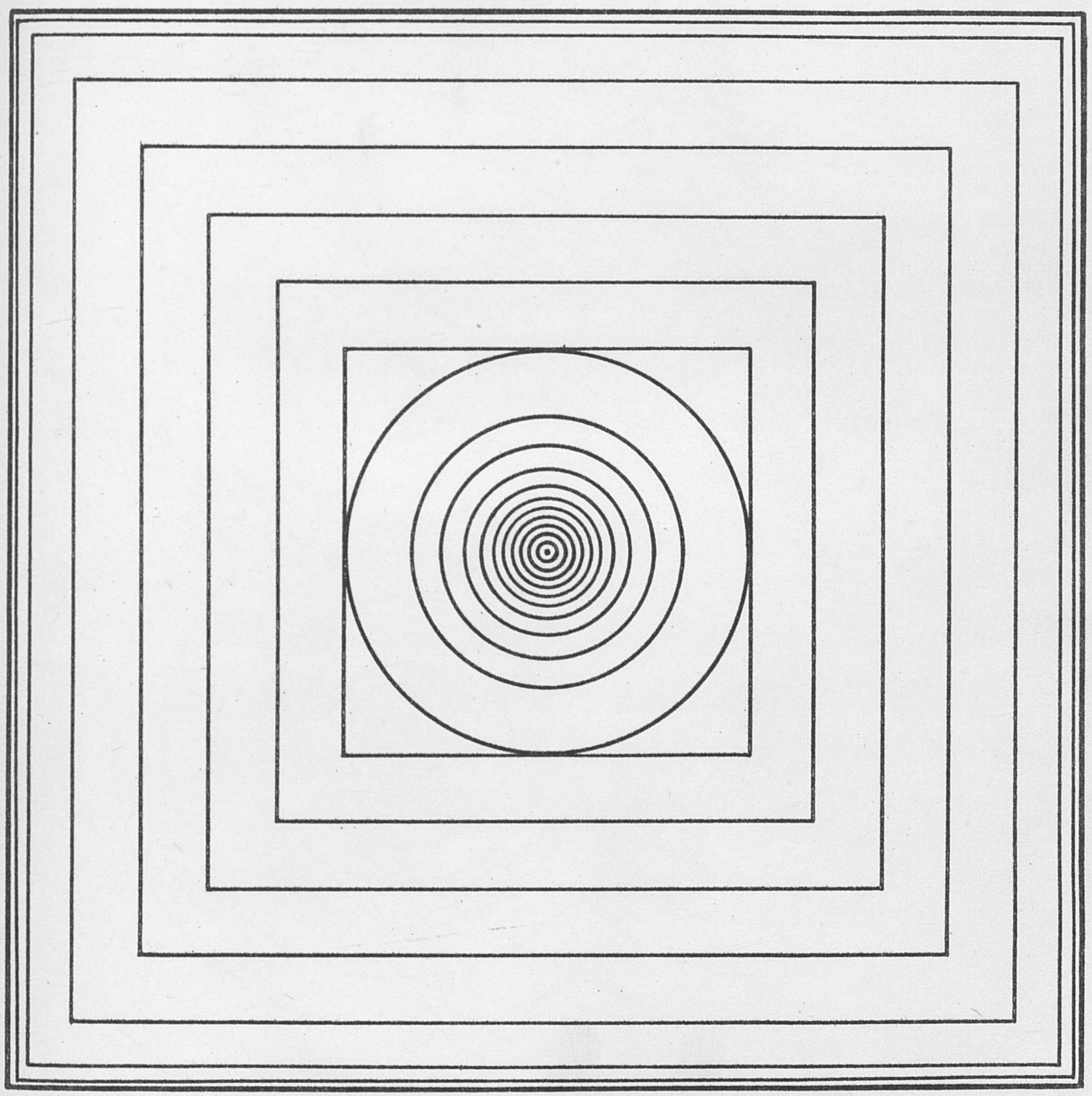


Evaporation from a Metal Surface Containing a High Concentration of Screw Dislocations at High Undersaturation (low p) and Low Temperature Giving Pitting at Screw Dislocations and Control of the Evaporation by Screw Dislocation Spirals

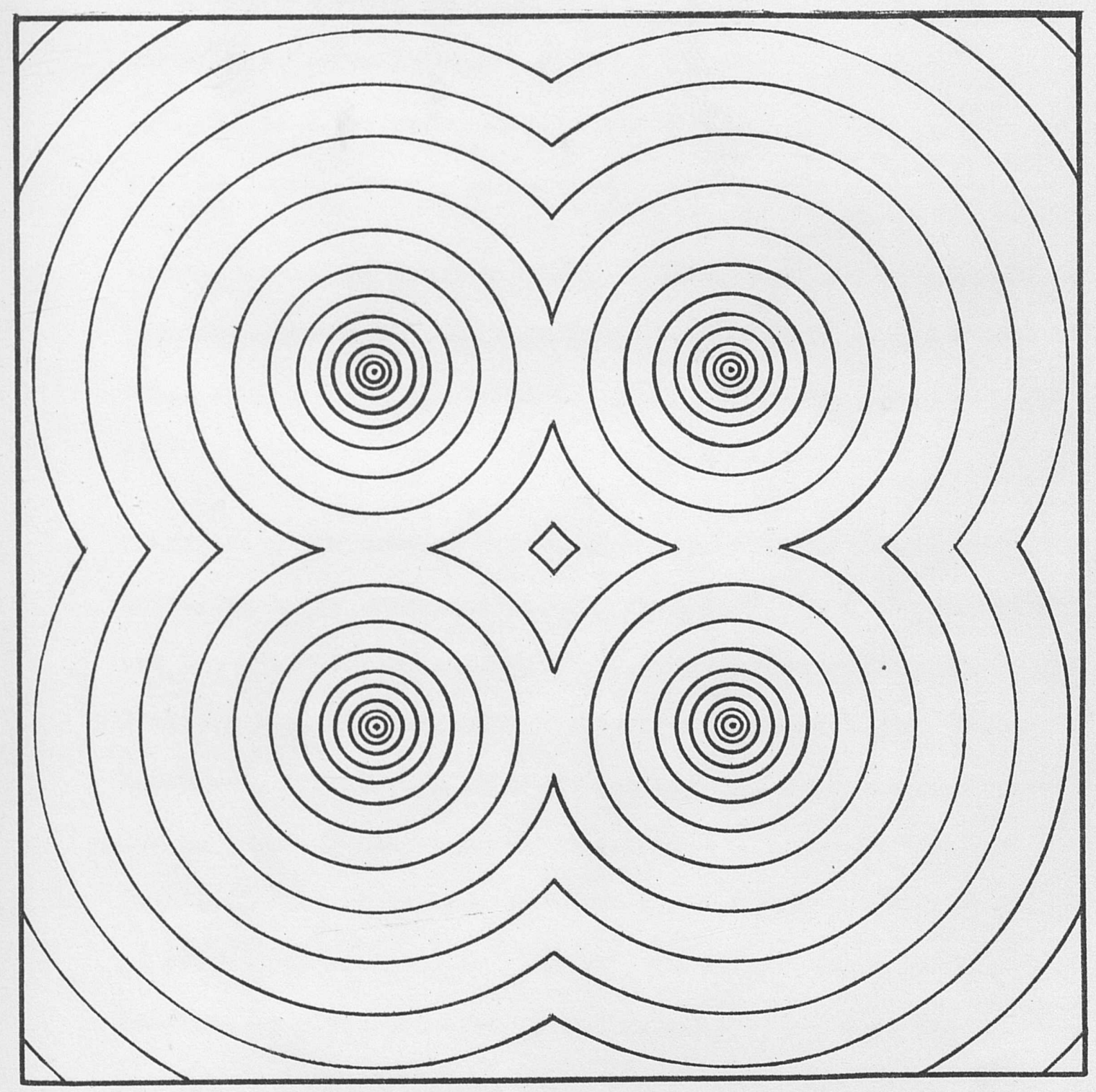


5. Macroscopic ledges and screw dislocations should cause positive deviations from the limiting expression for $\alpha$ only if they are present in high concentrations.

6. The expression for the evaporation coefficient of imperfect crystals is $\alpha=J / J c$, where $J c$ is given by equation ( $I$ ) and $J=\bar{J}+C_{2} p$ in which $\overline{J v}$ is given by equation (37). In this application of equation (37), $X^{\prime}$ is interpreted as the half distance between ledge sources such as pores, cracks, macroscopic ledges, etc.

7. In evaporation of metal crystals at large undersaturations, the terminal spacing of monat omic ledges emanating from screw dislocations should equal the terminal spacing of ledges emanating from crystal edges.

8. Considering the area of a perturbed region about a screw dislocation spiral at large undersaturations, such that $\lambda<\lambda_{0}$ in this region and the evaporation coefficient is greater than that given by the limiting law, together with the surface concentration of screw dislocations, a criterion is found for applicability of the limiting law in terms of the unperturbed fraction $\left(I-A_{0}\right)$ of the surface. From Table III it is seen that for a reasonably low concentration of screw dislocations, $10^{5}$ per $\mathrm{cm}^{2}$, and higher temperatures, the fraction of perturbed area is sufficiently small that the limiting law applies. On the other hand, higher concentrations of screw dism locations and lower temperatures lead to large deviations from the limiting law. It is proposed that this criterion may explain why 
measured evaporation coefficients approach the limiting value of $1 / 3$ at zero pressure in some experiments while in others the evaporam tion coefficient is found to be essentially unity.

9. At sma.11 undersaturations such that $19 \rho_{c}>\lambda_{0}$ the limiting law should apply regardless of temperature and concentration of screw dislocations.

10. The present considerations also provide a criterion for the degree of undersaturation which will lead to pitting at screw dislocations. At undersaturations sufficiently large that $19 \rho_{c}<\lambda_{0}$ and hence that true steady state evaporation from screw dislocations cannot occur, the flux from the region near the screw dislocation will be higher than elsewhere on the surface and pronounced pitting will occur. 


\section{EXXPERIMENT}

\section{A. Introduction}

In view of the numerous predictions made in the preceding theoretical treatment, it was felt that an experiment of a general survey nature would be the most fruitful approach to provide verification for the theory and to yield a foundation for further, more detailed research projects. Accordingly an experiment was undertaken in which the variables crystal perfection, orientation, pressure, and temperature were all controlled, enabling quantitative evaporation rates to be measured and the surface morphology to be studied metallographically. Specifically, the intent of the test was to detect evaporation ledge sources such as crystal edges, grain boundaries, dislocations, etc.: to note the extent of the effect of dislocations on surface morphology, evidenced as pits or spirals; and to compare evaporation rates from various single crystal surfaces and from polycrystals with that computed from the equilibrium vapor pressure assuming an evaporated coefficient of unity.

\section{B. Experimental Equipment and Techniques}

\section{Apparatus}

In the usual Langmuir type experiment ${ }^{(2)}$ in which a wiro specimen is heated in vacuun, there is no exact control of specimen shape and orientation as was desired in this experiment. Therefore the apparatus depicted in Figures 10 and 11 was designed. The one ome diemeter cyline drical silver specimens (K, Figure II) were seated such that only one circular surface was exposed in a graphite specimen holder $(J)$. The graphite holder was inserted to act as a damper for fluctuations in 
(1)-
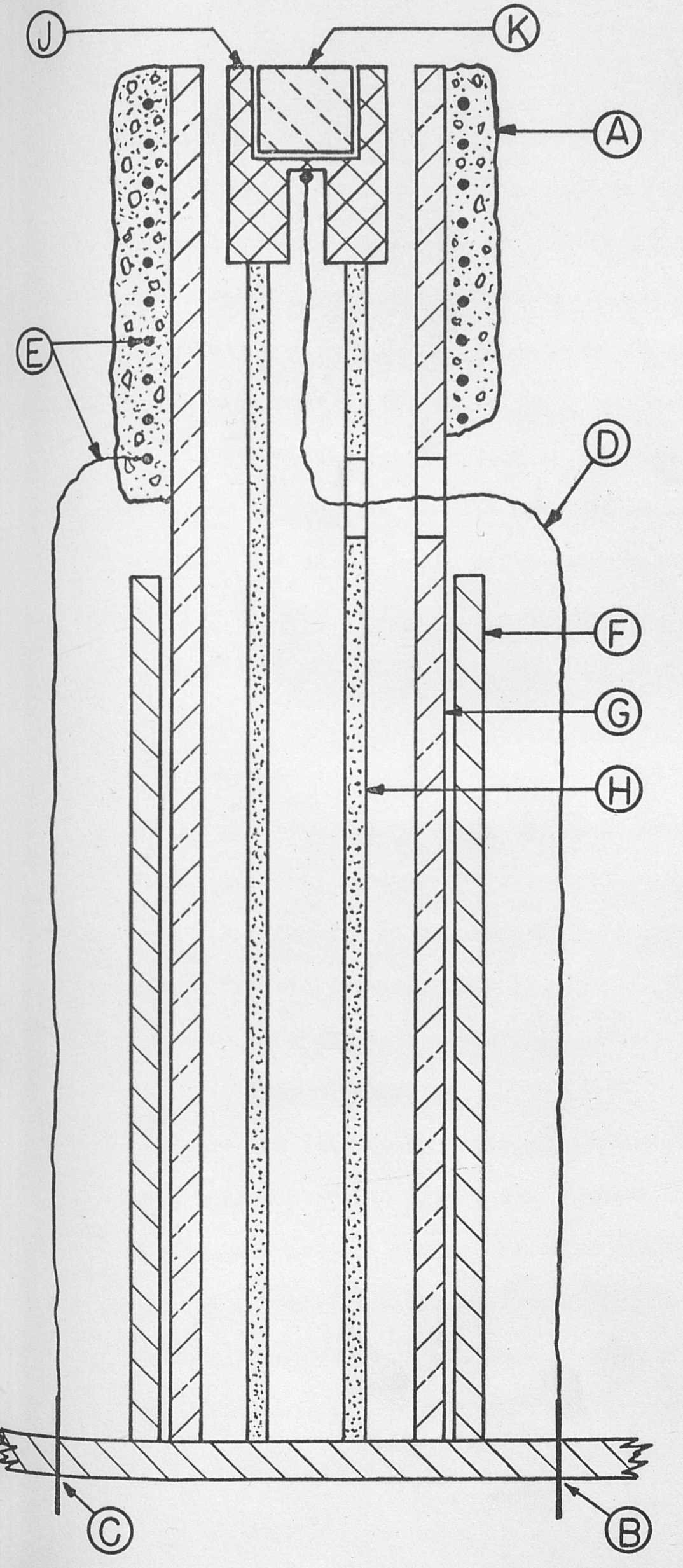

A-Alumina Cement

B - Thermocouple Leads Glass-Metal Seal

C-Power Leads Glass-Metal Seal

D- Thermocouple

E- Tungsten Heater

F-Furnace Support

G-Recrystallized

Alumina Tube $\mathrm{H}$ - Specimen Holder Support $J$ - Specimen Holder $\mathrm{K}$ - Specimen

DETAILED SCHEMATIC DIAGRAM OF SPECIMEN HOLDER AND FURNACE 
temperature arising due to gradients in the lower conductivity recrystallized alumina tube $(G)$. Temperature was controlled by a C. J. Tagliabue Controller sensing the temperature by a Chromel-alumel thermocouple (D) and controlling the current to the leads (E) by an on-off mercury relay in parallel with a shunt resistance. Power was supplied by two powerstats connected to the output of a constant voltage transformer. The heating elements were 15 mil tungsten wire wo und at 20 turns per inch on an $18 \mathrm{~mm}$ alumina tube and encased in recrystallized alumina cement. Due to the small dimensions of the furnace, non-inductive winding was not utilized, but the difficulty in temperature measurement thus arising was obviated by calibration of the temperature of the specimen.

Average evaporation rates were obtained by weighing the specimen before and after evaporating it for a given period of time at temperature. Continuous rate measurement was carried out by detecting the weight gain of a 3 cm diameter silver target (F, Figure 10) attached to a calibrated Nispan "C" microspring. The deflection of the microspring was measured by means of a Gaertner cathetometer.

\section{Vacurm Technique}

General vacuum technique is discussed by Dushman ${ }^{(52)}$. However, in the course of the experiment, various difficulties arose which are elucidated here for the reference of subsequent workers performing this type of experiment. Originally, stopcocks were inserted in the $18 \mathrm{~mm}$ pyrex. vacuum lines on the vacuum side of the furnace enclosure and before the oil diffusion pump so that portions of the system could be isolated to avert complete outgassing every time that a specimenwas removed or inserted. 
It was found that these stopcocks were a leak source below a $10^{-5} \mathrm{~mm}$ of mercury vacuum. This was presumably due to gas dissolved in the stopcock grease which outgassed when the stopcock was turned. The minimum attainable pressure in the system was decreased by reducing the total number of grease (stopcock) joints and glass-inetal seals in the system. The cold cathode gauge was found to be more satisfactory than an ionization gauge for pressure measurement in the range $10^{-4}-10^{-7} \mathrm{~mm}$ of mercury since it requires no outgassing and does not become contaminated. The additional pumping speed that could be attained by the use of an ionization gauge was negligible in this pressure range.

Leak detection with a spark tester is satisfactory to detect leaks to $10^{-7} \mathrm{~mm}$ pressure for the glass portions of the system. However, it was necessary to insert a cold trap between the furnace chamber and the gauge so that the metal-glass seals could be tested for leaks by spraying acetone on the seals. The acetone passes into the system through any leak present, blocking air flow through the leak, and condenses out in the trap causing a rapid drop in the pressure reading of the gauge.

Dynamic vacuums were obtained in the course of the experiments. At room temperature the pressure obtained was 0.5 to $1.2 \times 10^{-7} \mathrm{~mm}$. After outgassing the furnace for twenty-four hours at $400^{\circ} \mathrm{C}$, the pressure obtained at testing temperat ures of $600^{\circ} \mathrm{C}, 700^{\circ} \mathrm{C}$, and $750^{\circ} \mathrm{C}$ was 0.5 to $8.0 \times 10^{-5} \mathrm{~mm}$.

3. Specimen Selection and Preparation Silver was selected for this test for the following reasons: (a) silver evaporates essentially monatomically as a solid although the liquid may evaporate with $\sim 0.1 \%$ dimer $(53,54,55)$, (b) silver could be 
obtained in a highly pure form, (c) single crystals of silver could feasibly be made, (d) silver has a high conductivity and low emissivity so that a uniform temperature could be maintained in vacuum, (e) silver has a relatively high vapor pressure, and (f) silver oxide has a high dissociation pressure, $\sim 200 \mathrm{~mm}$. of mercury at $600^{\circ} \mathrm{C}$.

Oriented silver single crystal spheres and cylinders of $99.99 \%$ purity were obtained from the Virginia Institute of Scientific Research. Other silver single crystals of $99.99 \%$ purity were furnished by Dr. P. G. Shewmon of the Westinghouse Research Laboratories. Polycrystalline specimens were prepared from granulated silver of $99.99 \%$ purity obtained from the Vermon-Bershoff Company.

The oriented single crystals were tested for orientation and degree of perfection (see Appendix II) and evaporated as received. Polycrystalline specimens were machined to size, polished to 0.5 micron diamond. paste polish, annealed in argon for one hour at $800^{\circ} \mathrm{C}$ to stabilize the grain size and evaporated.

All specimens were degreased in acetone prior to weighing or testing. 4. Calculation of Results

a. Average Evaporation Rate

The average evaporation rate was obtained by dividing the total weight loss of the specimen by the area evaporating and by the time elapsed in the test. The corresponding rate based on the equilibrium vapor pressure is given by equation (2).

b. Spherical Specimen Evaporation Rate The weight loss of a spherical crystal as a function of time is derived in Appendix III and is expressed as: 


$$
\begin{gathered}
m=-\frac{4 \pi}{3 \bar{\rho}^{2}}\left(\frac{P M}{\sqrt{2 \pi M R T}}\right)^{3} t^{3}+\frac{4 r_{0} \pi}{\rho}\left(\frac{P M}{\sqrt{2 \pi M R T}}\right)^{2} t^{2}-4 \pi r_{0}^{2}\left(\frac{P M}{\sqrt{2 \pi M R T}}\right) t \\
+\frac{4 \pi r_{0}^{3} \bar{\rho}}{3} .
\end{gathered}
$$

where $\mathrm{m}=$ wt. loss in grams, $\bar{\rho}=$ density, $M=$ molecular wt., $r_{0}=$ initial radius of sphere, and $t=$ time.

\section{c. Evaporation Rate from Target Weight Gain}

The formula for the evaporation rate from a circular surface besed on the impingement or a parallel circular surface with the centers of the two surfaces lying on a perpendicular to the surfaces is analogous to the formula for radiant heat transfer between two such surfaces ${ }^{(56)}$

$$
\frac{d m}{d t}=J \frac{\pi}{2}\left[b^{2}+a^{2}+k^{2}-\sqrt{\left(b^{2}+a^{2}+k^{2}\right)^{2}-4 a^{2} b^{2}}\right]
$$

where $\frac{d m}{d t}$ wreight gain of target per unit time, $J$ I rate of evaporation from specimen, $a=$ radius of secimen, $b=$ radius of target, and $k=$ the perpendicular distance between the two surfeces. This formula is developed by considering the impingement on a differential area of the target of the fraction of the flux emitted from a differential area on the source and integrating over the total area of both surfaces.

\section{Estimated Accuracy of Measurement}

a. Spring Calibration

The Nispan "C " microspring, $5 \mathrm{mil}$ wire wound on a halfoinch tube and annealed one hour at $850^{\circ} \mathrm{C}$ in argon, was calibrated with weights of from ten to two hundred milligrams. Fach deflection measured with cathetometer was taken as an average of ten readings with a maximum doviation of \pm .0004 inches. The spring constant calculated from these 
deflections was $10.532 \pm 0.002$ inches $/ \mathrm{g}$ or $0.09496 \pm 0.00001 \mathrm{~g} /$ inch.

b. Weight Measurement

Weighings were taken on a Christian Becker chain balence rem producible for a given weighing to $\pm 0.00007 \mathrm{~g}$. The balance was calibrated before each weight measurement to a control weight of the same approximate weight as the test specimens. The control weight weighed $8.0269 \mathrm{~g}$. Weight differences are thus felt to be accurate to $\pm 0.0001 \mathrm{~g}$. c. Time

Since the heating rate in raising the specimen to temperature was c. $35^{\circ} \mathrm{C}$ per minute near the testing temperature, and since all quantitative tests at $750^{\circ} \mathrm{C}$ were c. 700 minutes in length, the zero time was taken with essentie.l ly negligible error as the time when the specimen reached temperature. The stopping time was measured in a similar mamer. At $850^{\circ} \mathrm{C}$ the tests were about 100 minutes duration, introducing an error approaching $0.5 \%$.

\section{d. Length Dimensions}

The area of the evaporating specimen was measured to 0.005 square cm. or c. $0.4 \%$. The area of the target was s.ccurate to $0.8 \%$. The distance between source and target was maintained to $\pm 0.5 \%$

\section{e. Vocurm}

The pressure in the first hour of the exp erimental rums dropped from $4 \times 10^{-4} \mathrm{~mm}$ to $8 \times 10^{-5}$ necessitating the application of the correction factor discussed in Appendix IV to the results of the spring deflection measurement. Due to the application of this factor to the average pressure over an interval between successive deflection measure ments, there was an error of the order of $10 \%$ in the first hour of time 
in the continuous deflection measurement, the error dropping to $\sim 1 \%$ after that $t$ ime period. Further, the readings were corrected for attainment of thermal equilibrium by subtracting the deflection of the spring during a blank run. It is emphasized that these corrections apply only to the continuous measurement and not to the average rate measurement.

\section{f. Temperature}

The controller maintained the tempe rature of the specimen to $\pm 0.5^{\circ} \mathrm{C}$ over short time periods. However, due to variation of the standardization of the controller with time and ambient temperature, the error in reproducibility during temperature control was $\pm 1.0^{\circ} \mathrm{C}$, maintained at this level by menual standardization of the controller. An additional error of $0.1^{\circ} \mathrm{C}$ arose due to the accuracy of standardizing the reference potentiometer. The thermocouple was calibrated to the ice point and to the melting point of lead. The drift of the thermocouple over the series of quantitative rate measurements was less than $I^{\circ} \mathrm{C}$.

The temperature of the specimen in situ was determined by measuring the melting point of silver. Temperatures used in pressure calculations were corrected to this standard value. During the tests, the absolute temperature was checked by measuring the melting point ${ }^{(57)}$ of potassium chloride crystals on the surface of the metal specimen under an argon atmosphere. The final estimated error in temperature is $\pm 1.5^{\circ} \mathrm{C}$ in reprom ducibility, $\pm 3^{\circ} \mathrm{C}$ in absolute value.

g. Remevaporation of Condensed Silver

The temperature of the target and of the walls of the furnace enclosure, both sites where silver condensed, did not exceed $200^{\circ} \mathrm{C}$. At this temperature the vapor peessure of silver is about $10^{-22} \mathrm{~mm}$. of 
mercury $(54,58)$ so that re-evaporation of the silver wes negligible. Also, the condensation coefficient of silver impinging on silver is unity at this temperat ure ${ }^{(59)}$ so that no silver was reflected back to the source.

\section{h. General}

Since the results are in general too sparse to permit a statistical evaluation of the error, the result s are presented with an estimated maximum error based on the various possible sources of error enumerated above. It is evident from the above discussion that the average evaporation rate measurement is much more precise than the con tinuous measurement by means of the microspring. The only really significant source of error in the former is the temperature. The precision of the average evaporation rate measurements, and thus the result s reported as pressure and $\alpha$, is $\pm 10 \%$

\section{Experimentel Results and Discussion \\ 1. Surface Morphology}

\section{a. Initial Pitting of Spheres}

Spherical single crystals of silver with known orientations were evaparated at $623^{\circ} \mathrm{C}$ and $727^{\circ} \mathrm{C}$. After one hour at $623^{\circ} \mathrm{C}$, pits with a density of about $2 \times 10^{6}$ per $\mathrm{cm}^{2}$ were observed orer the entire surface. Pits at the $\{111\}$ poles had intersections with the crystal surface that were hexagonal or triangular in shape, with the sides of the polygons lying along close packed $\langle 110\rangle$ directions as shown in Figure 12. Pits at the $\{100\}$ poles had square surface intersections, with the sides parallel to $\langle 110\rangle$ directions as shown in Figure 13. At intermediate positions facets with faces perpendicular to $\{111\}$ tended to form, with 
pits characteristic of $\{11]\}$ forming on the facet sur fla ce. An example of this behavior is depicted in Figure 14. This figure also shows some of the small angle boundaries which appeared with a very low frequency on the surface.

After longer periods of evap oration at $623^{\circ} \mathrm{C}$, the pits started to interact and lose the regularity of outline characteristic of their initial appearance. This is shown in Figures 15 and 17 and in the sequence in Figure 16. As cen be seen in Figure 16, the bottom of the pit, initially flat, tends also to break up with increasing evgp oration. This behavior is consistent wh th the usual observation involving interfacial free energy that when the surface to volume ratio of the crystal or void in question is large, the surface tends to assume a shape dominated by the interfacial free energy term rather than the bulk free energy (27). When as frequently occurs at temperatures somewhat below the melting point, there is a sharp cusp in the interfacial free energy versus orientation plot at orientations corresponding to low index planes, then the interfecial free energy controlled shepe will be one in which the boundary fraces are low index planes. Direct corroboration of this result is also found in the work of Evans $(60)$ on the thermal etching of titanium.

b. Correlation Between Etch Pits and Dislocations As described in Appendix II, the density of dislocations on surfaces of $\{111\}$ and $\{100\}$ orientation corresponding to Figures 12 and 13 is $\sim 10^{8}$ per $\mathrm{cm}^{2}$ as compared with an observed density of pits of 2 $\times 10^{6}$ per $\mathrm{cm}^{2}$. On the other hond, a flat $\{110\}$ surface gires rise to pits of density of $\sim 10^{8}$ by both methods, see Figure 26. 


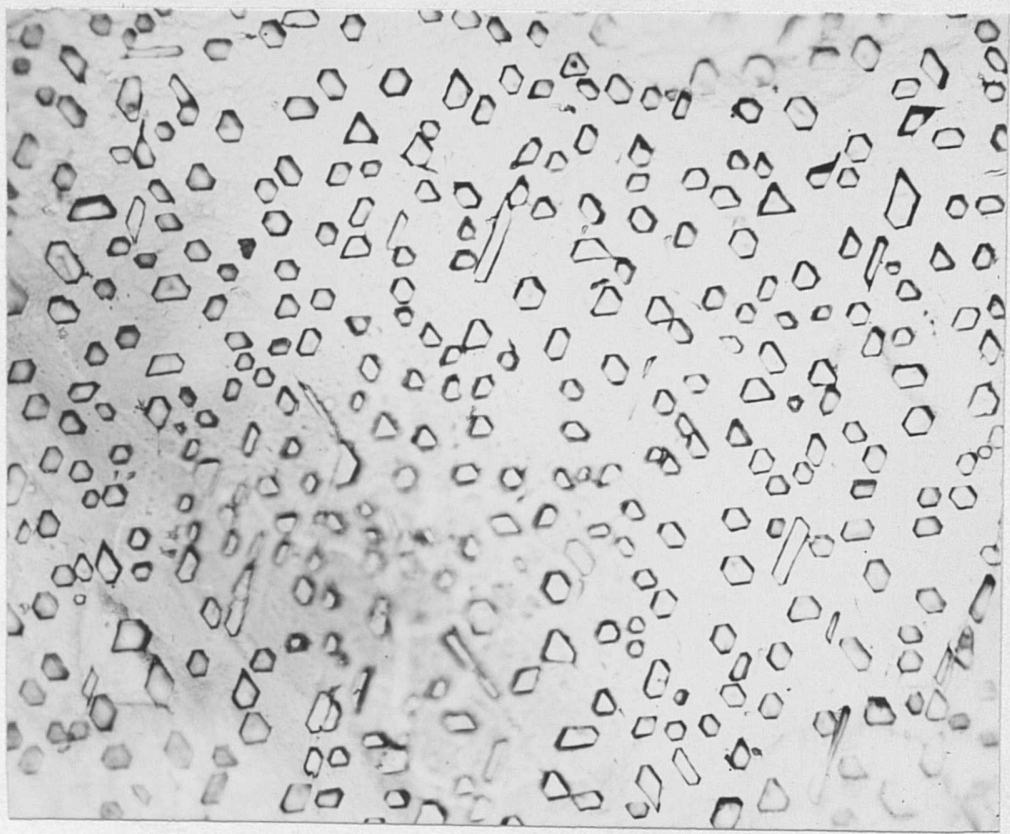

Figure 12. $\{111\}$ surface after evaporation at $623^{\circ} \mathrm{C}$ for 90 min., spherical single crystal, 500x.

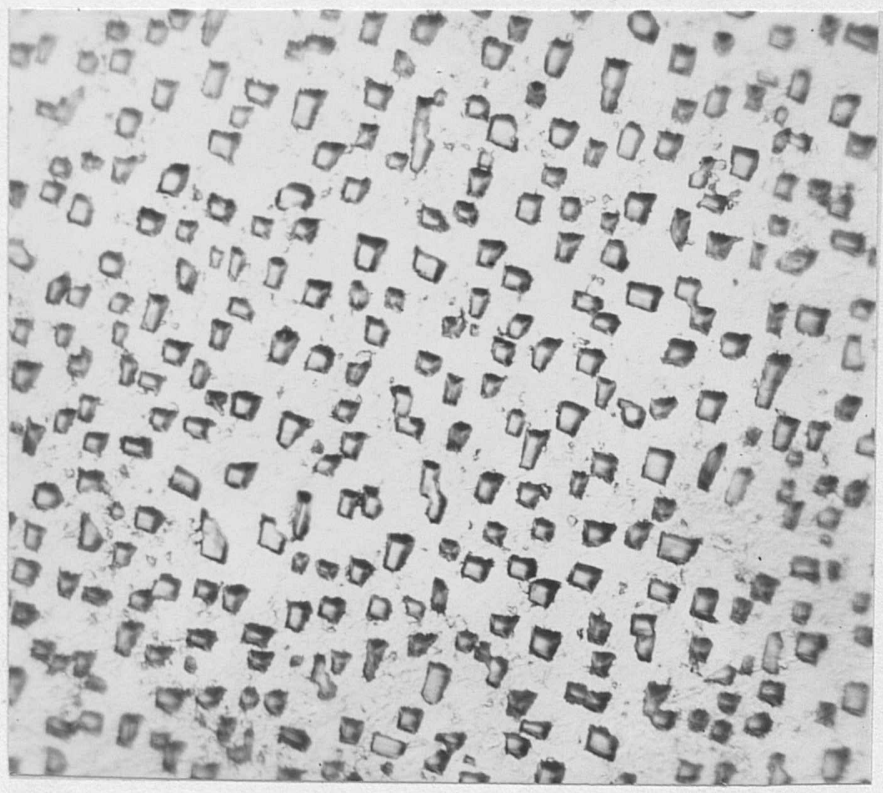

Figure 13. $\{100\}$ surface after evaporation at $623^{\circ} \mathrm{C}$ for 90 min., spherical crystal, 500x. 


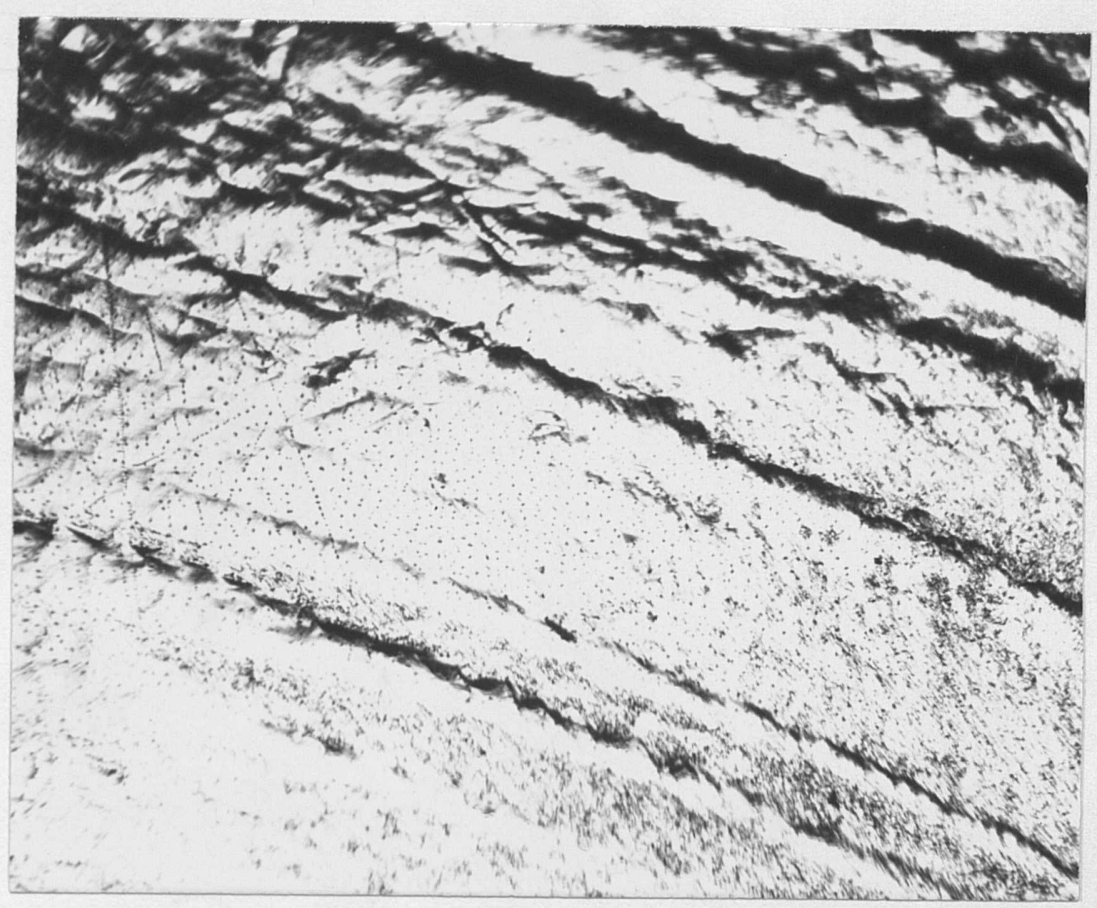

Figure 14. Intermediate position botween \{111\} and $\{100\}$ after evaporation at $623^{\circ} \mathrm{C}$ for 270 min.? spherical single crystal, $50 \mathrm{x}$.

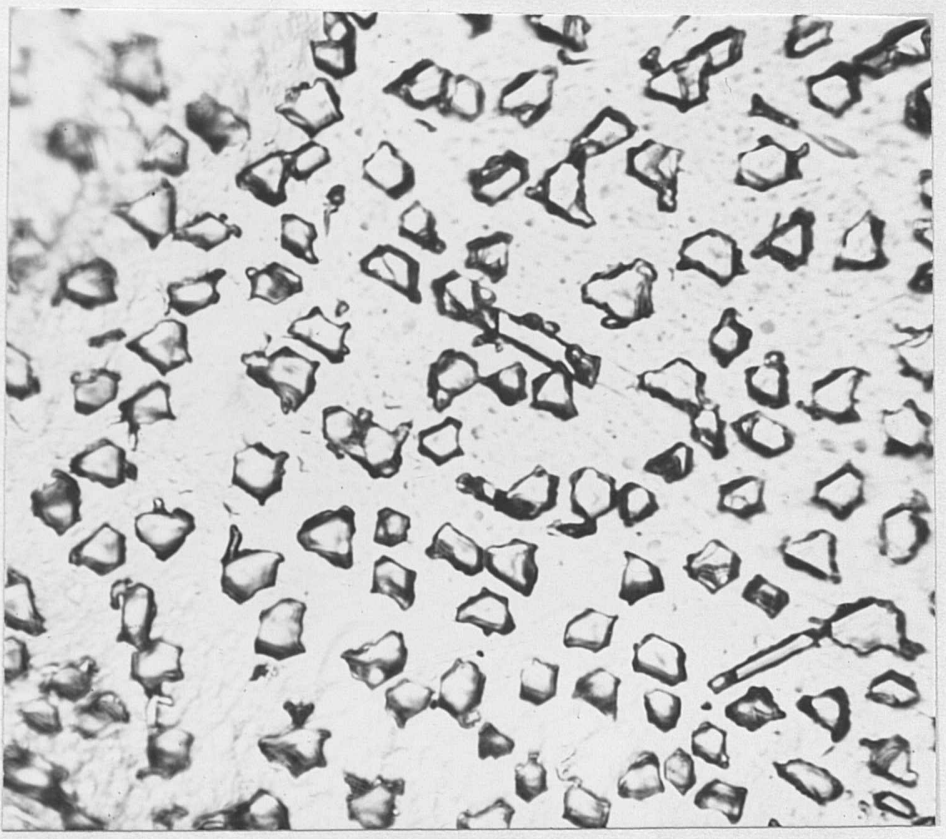

Figure 15. $\{111\}$ surface after evaporation for $325 \mathrm{~min}$. at $623^{\circ} \mathrm{C}$, spherical single crystal, 1000x. 


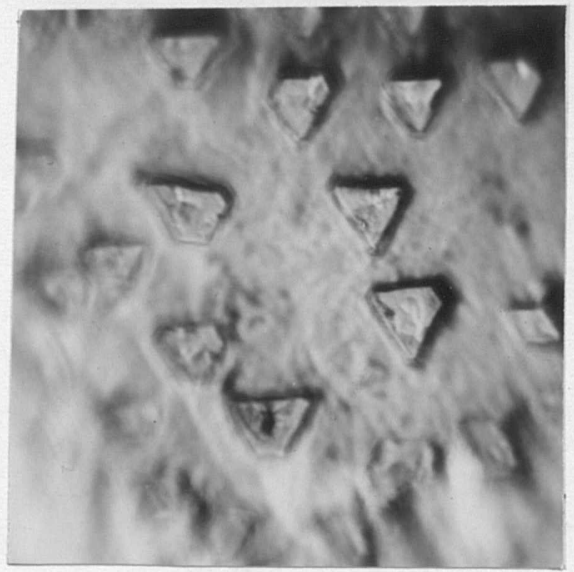

a. 190 min., base.

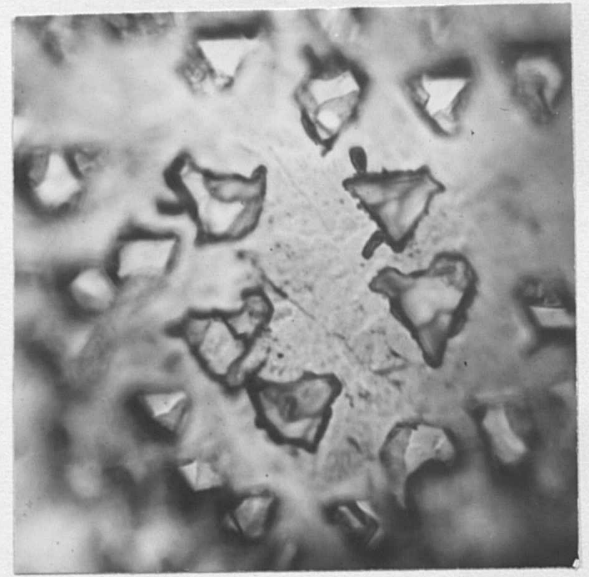

c. 325 min., base.

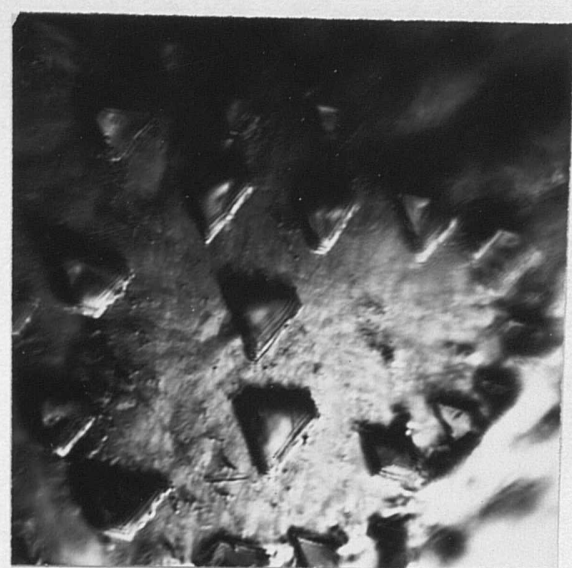

b. 190 min., surface.

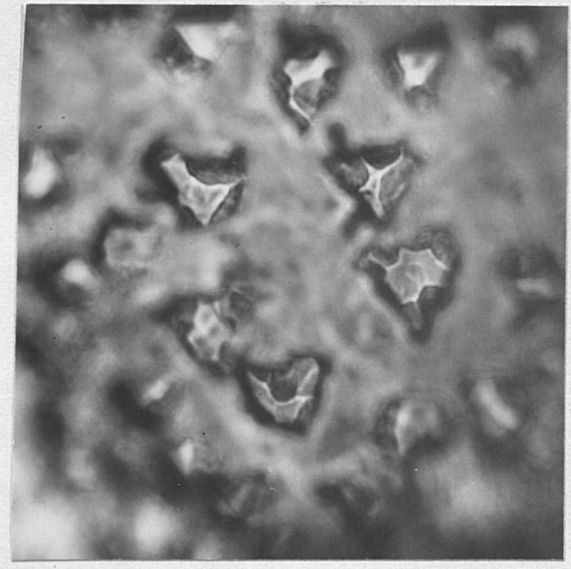

d. 325 min., surface.

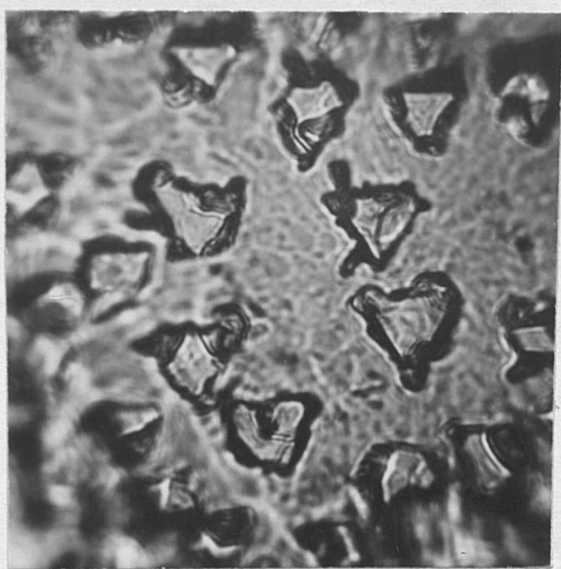

c. 515 min., base.

Figure 16. $\{111\}$ surface after evaporation at $623^{\circ} \mathrm{C}$ for the times indicated and with the focus on the surface or bottom of the pit as shown, spherical single crystal, 1000x. 


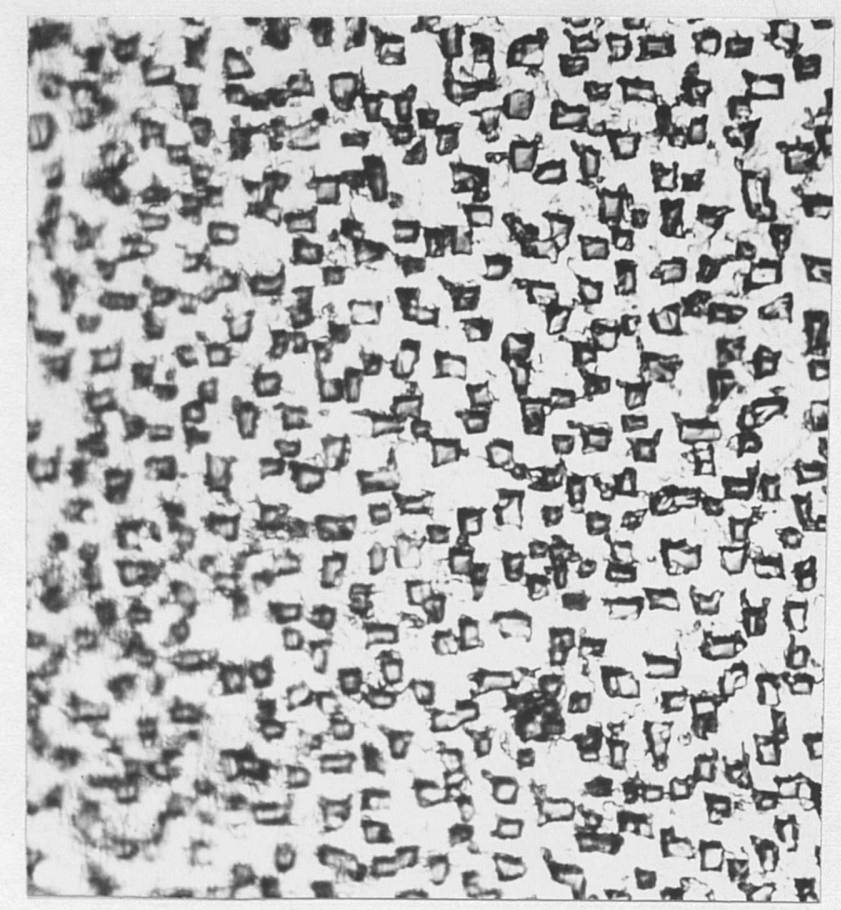

Figure 17. $\{100\}$ surface after evaporation at $623^{\circ} \mathrm{C}$ for 325 min., spherical single crystal, $500 \mathrm{X}$.

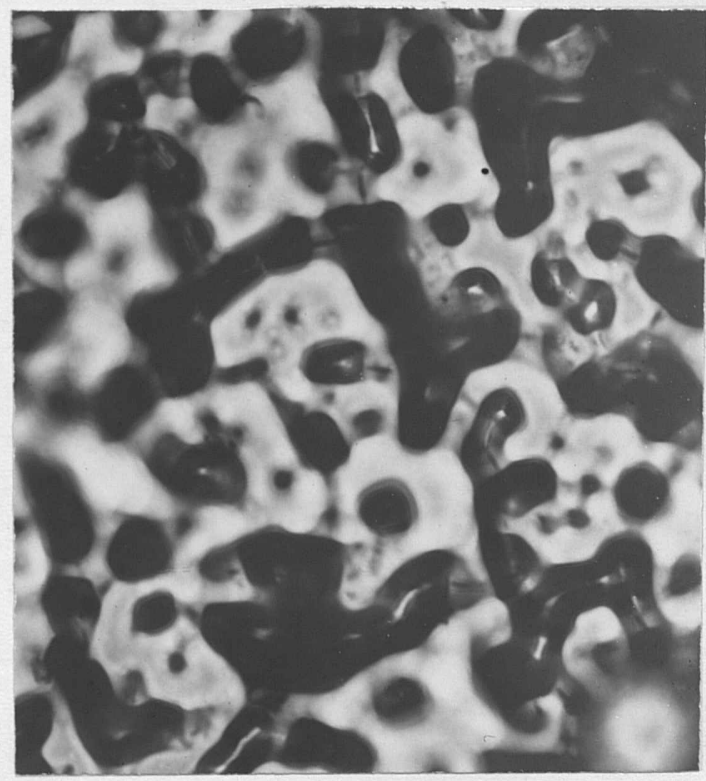

Figure 18. Small pits wi. thin macro pits on surface after evaporation at $847^{\circ} \mathrm{C}$ for 105 min., polycrystal, $1000 \mathrm{x}$. 
Vogel et al ${ }^{(61)}$ showed that the number of etch pits at low angle boundaries, developed by chemical etching of germanium, corresponded closely to the number calculated from grain boundary angles. Further evidence of this nature was found by Meleka ${ }^{(62)}$, Hibbard ${ }^{(63)}$ and others. However, Ellis $(64)$ recently demonstrated that pits presumably due to edge dislocations gave rise to large etch pits in germanium single crystals, while smaller pits, corresponding to screw dislocations, were also observed.

Hendricks on and Nechlin ${ }^{(65)}$ thermally etched silver crystals in 1 part $\mathrm{O}_{2}$ and 9 parts argon at $600^{\circ} \mathrm{C}$ and found $2 \times 10^{6}$ etch pits per $\mathrm{cm}^{2}$. By analogy with the above work, they assumed that this represented the dislocation density. The present work indicates that this is not the case with silver. The appearance of the large pits on close packed surfaces but not on flat $\{110\}$ surfaces, coupled with the instability of the larger index surfaces of the spherical crystals, suggests that the macroscopic pits are associated with the mechanism of evaporation from low index planes. The large pits would form at sites where dislocations with a large component of their Burger's vector perpendicular to the surface intersect the surface. Smaller pits would be dislocations with a smaller screw component normal to the surface. These latter dislocations could be partial dislocations since the presence of twinning in silver suggests that the formation of extended dislocations in silver is possible $(66)$. The dislocations intersecting the $\{110\}$ surfaces tend to all have the same pitting effect, probably due to strain energy, since this plane tends to be inactive per se in evaporation, not becoming active until the plane has broken up into close-packed plane facets. 
Some evidence for the behavior suggested in this paragraph is shown in Figure 18, where, after evaporation at $843^{\circ} \mathrm{C}$, fine pits were observed within the larger pits.

An alternative explanation that the pits are associated with doubleBurger's vector dislocations can be discarded since the number of these dislocations can be related to the number of single vector dislocations by the ratio of the exponent of the Burger's vector squared subject to the assumption that elasticity equations apply. For a typical dislocation energy of $\sim 3 \mathrm{e} \cdot \nabla \cdot$ per atom spacing the ratio of concentrations of single to double Burger's vectors is $e^{360}$, suggesting that double Burger's vectors can be discounted.

Nevertheless, the treatment discussed in Section I-C will still apply to real evaporation kinetics with the condition that only the concentration of active dislocation ledge sources be considered.

A final point of interest is to note that in the chemical etch treatment to reveal dislocations and in thermal etching such as that of Machlin ${ }^{(65)}$, it is frequently necessary to add an impurity element to "decorate" the dislocation-surface intersections (42) whereas in the present work it sufficed to maintain a high vacuum to reveal the etch pits.

c. Instability of High Index Planes

It was found that, after one hour $a_{\text {shape, }}\{100\}$ and $\{111\}$, had other than those present in the Gibbs-Wulff shape, $\{100\}$ and $\{111\}$, had broken up into facets of $\{111\}$ or $\{100\}$ orientations. This was revealed by the reflection of visible light from these orientations. The $\{111\}$ reflection was generally stronger than the $\{100\}$ reflection. In addition, 
metallographic observation revealed that internediate positions on the spherical surface showed $\{111\}$ facets as indicated in Figure 19.

Several possibilities exist to explain the instability of high index planes. For small crystals where surface free energy is a criterion in evaporation kinetics, high index planes will quickly be annihilated by accretion of atoms by vapor transport and by surface diffusion from low index planes. In the evaporation of larger crystals, the formation of macroscopic steps or facets of low index planes again eliminates high index plane surfaces during evaporation. The mechanisn of formation of these macroscopic steps is not clear as will be discussed later.

\section{d. Limiting Surface Morphology}

The initial surface morphology of oriented single crystal cylinders of $\{100\}$ and $\{111\}$ surface orientation was identical with that observed on the spheres as exemplified in Figure 20. After Ionger periods of evaporation, during which evaporation approached a limiting rate as will be discussed in the next section, $\{100\}$ surfaces becarne dominated by ledges emanating from the original square etch pit sites on the surface and assumed the structure shown in Figures 21 and 22. $\{111\}$ surfaces assumed the morphology depicted in Figures 23-25. It can be seen that the pits developed were very shallow in nature. No macroscopic ledges are present on these $\{111\}$ surfaces, contrary to the case of the $\{100\},\{110\}$, and higher index surfaces. This, in addition to the evidence of strong light reflection from $\{111\}$ poles on these non$\{111\}$ surfaces suggests that the formation of macroscopic ledges is associated with a break up into $\{111\}$ facets. 


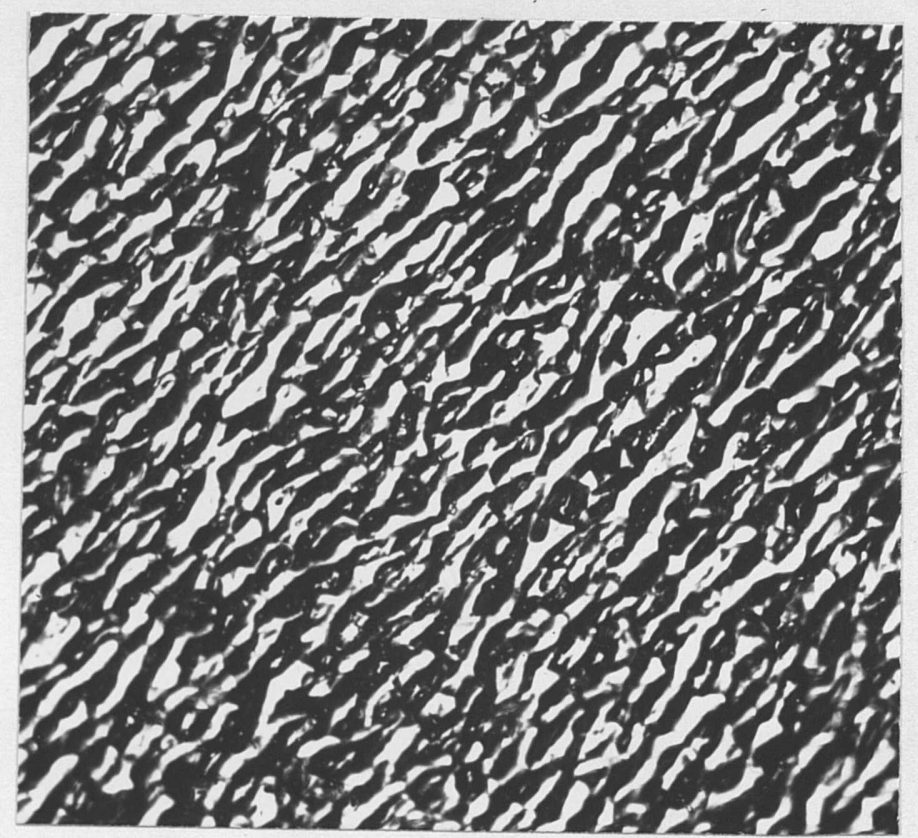

Figure 19. Intermediate surface between $\{111\}$ and $\{100\}$ showing $\{111\}$ facots, evaporated at $727^{\circ} \mathrm{C}$ for 558 min., spherical single crystal, 200x.

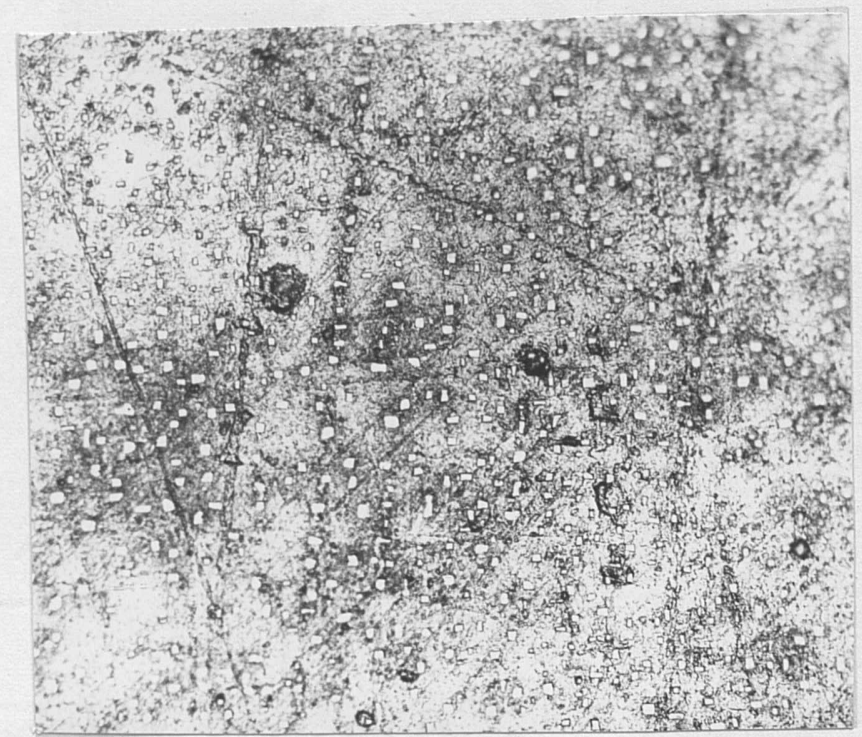

Figure 20. $\{100\}$ surface evaporated at $744^{\circ} \mathrm{C}$ for 50 min., cylinder single crystal, $200 \mathrm{x}$. 


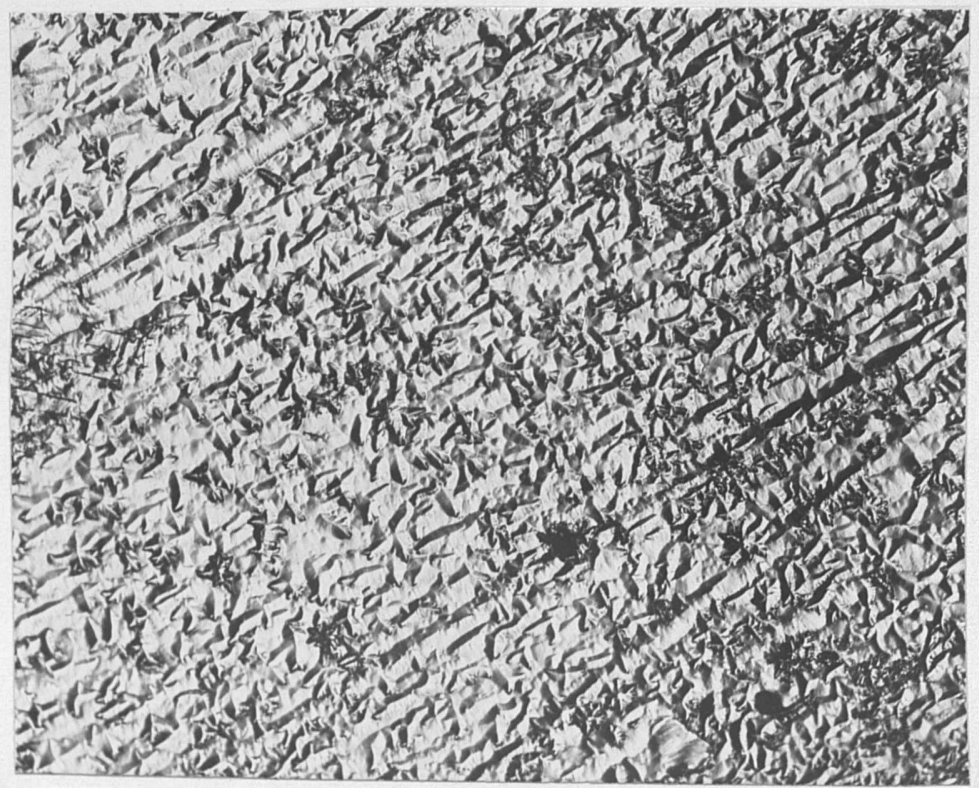
Figure 21. $\{100\}$ surface after evaporation at $744^{\circ} \mathrm{C}$ for 770 min., cylinder single crystal, $200 \mathrm{x}$.

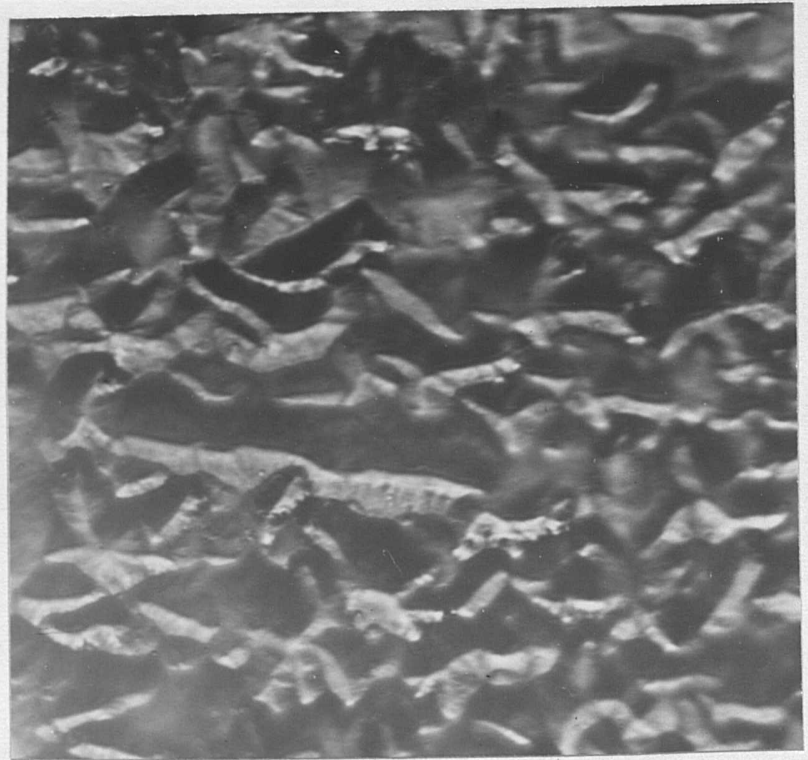

Figure 22. $\{100\}$ surface after evaporation at $744^{\circ} \mathrm{C}$ for 770 min., cylinder single crystal, oblique lighting, $1000 \mathrm{x}$. 


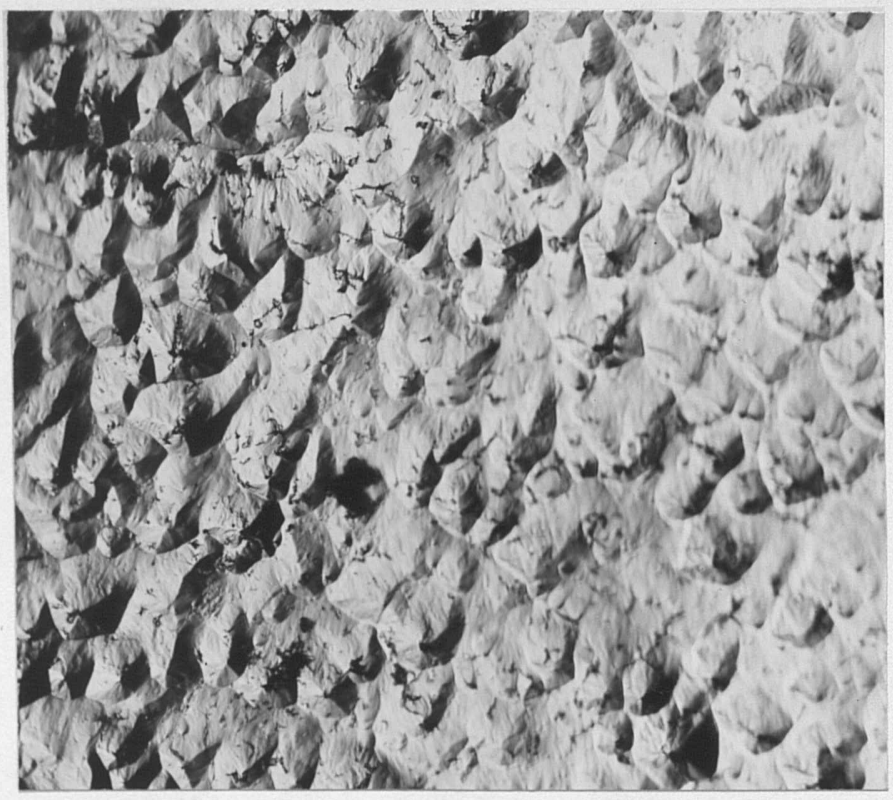

Figure 23. $\{111\}$ surface after evaporation at $744^{\circ} \mathrm{C}$ for 690 min., cylinder single crystal, $200 x$.

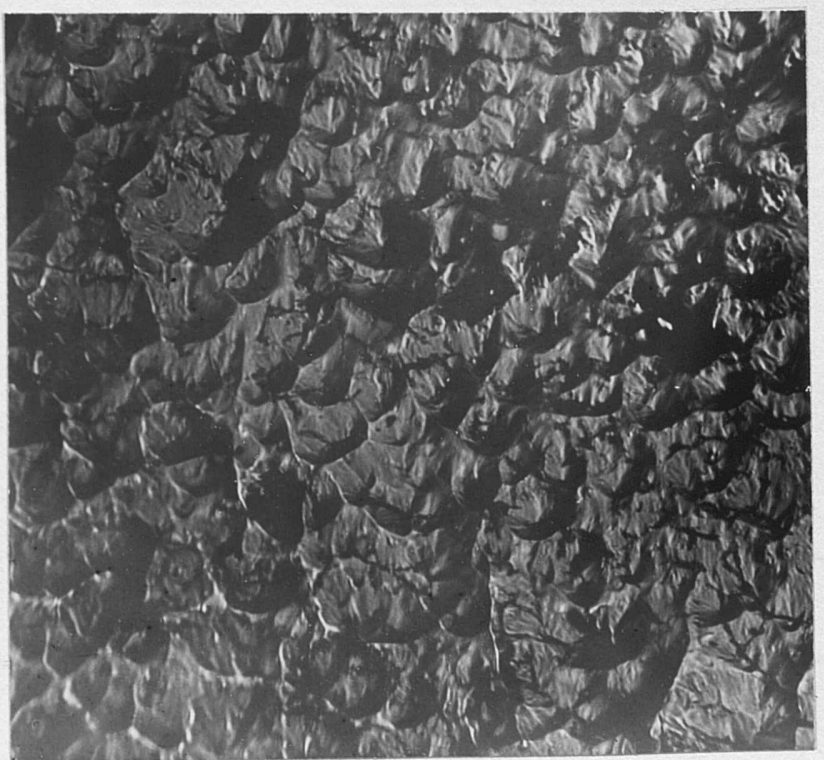

Figure 24. $\{111\}$ surface after evaporation at $744^{\circ} \mathrm{C}$ for $690 \mathrm{~min}$, cylinder single crystal, oblique lighting, 200x. 


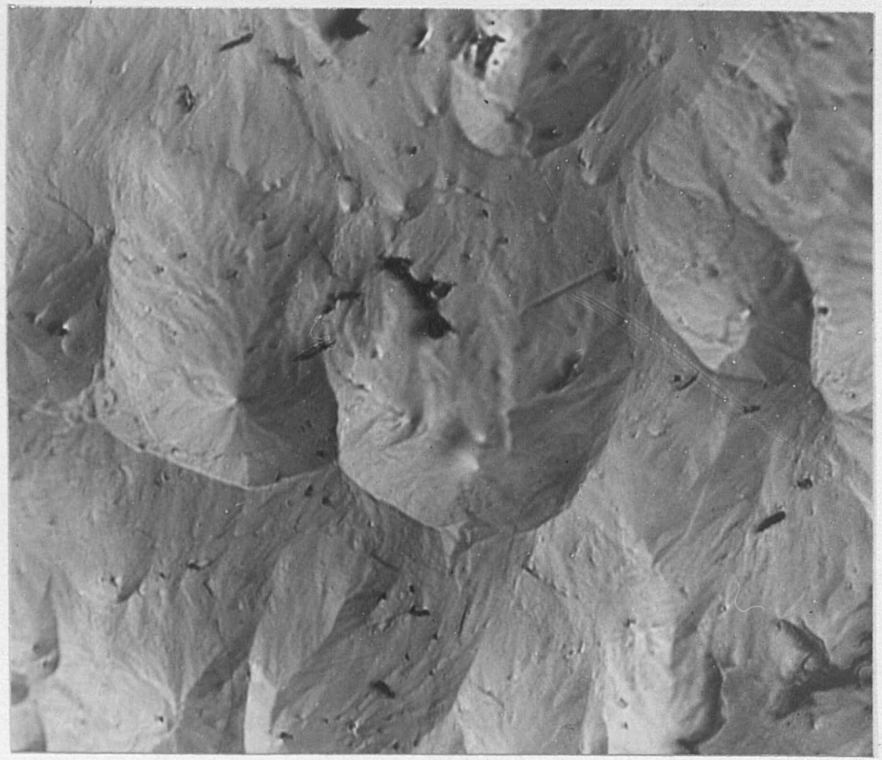

Figure 25. $\{111\}$ surface after evaporation at $744^{\circ} \mathrm{C}$ for 690 min., cylinder single crystal, 1000x.

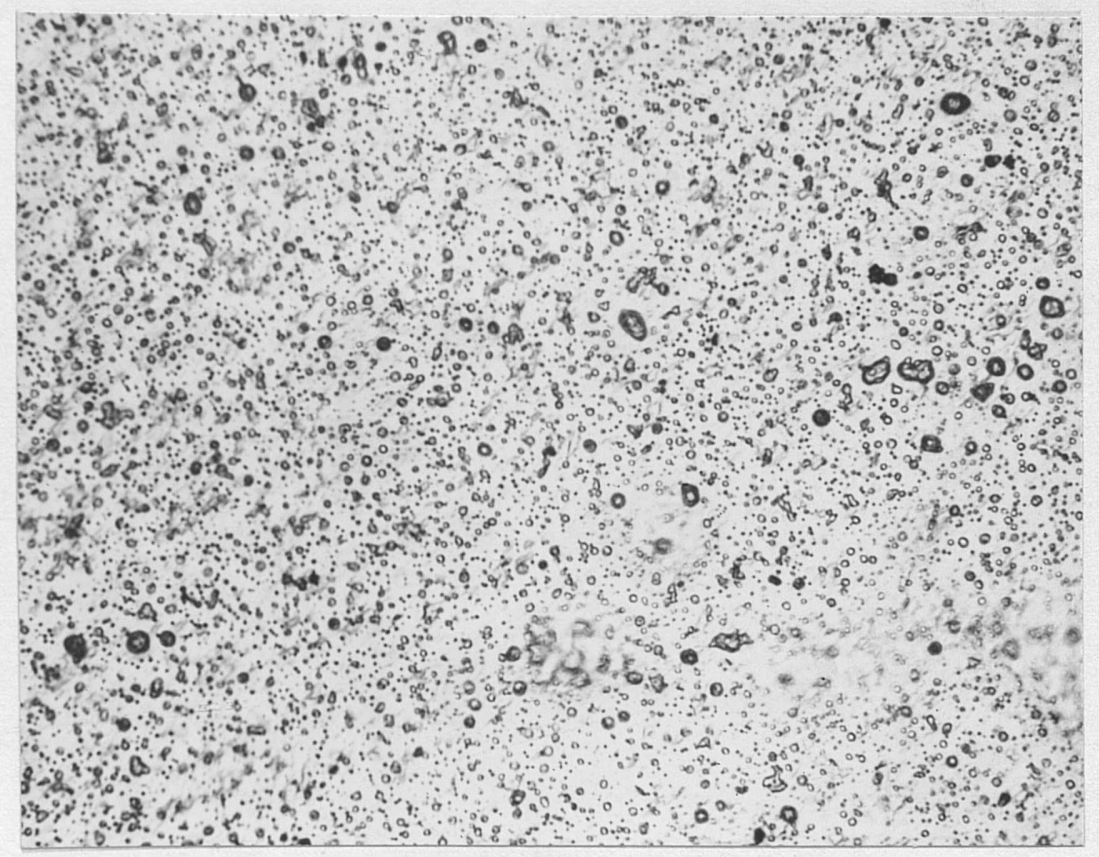

Figure 26. \{111\} surface after evaporation at $644^{\circ} \mathrm{C}$ for 60 min., cylinder single crystal, $1000 \mathrm{x}$. 
\{110\} surfaces, as mentioned above, initially show fine pits as demonstrated in Figure 26. Again, after further evaporation, the surface tends to break up into $\{111\}$ facets as shown by light reflection and in the sequence of Figures 27-29. Figure 29 reveals the presence of macroscopic steps on the facets by the technique of Verma (42) of using oblique lighting slightly out of focus.

\section{e. Grain Boundaries as Ledge Sources}

Although most of the ledges active in the evaporation of silver emanated from surface-dislocation sites, it was established, as shown in Figure 30, that grain boundaries did act as sources for ledges during evaporation into a high vacuum. This was suggested by the work of Mullins (67) who developed a detailed analysis of thermal grooving under smaller undersaturations. In the course of the investigation, occasionally a screw dislocation pit was found emanating from a grain boundary as set forth in Figure $31^{*}$.

Macroscopic ledges, issuing from a grain boundary of a chromium sample that had been thermally etched at $1250^{\circ} \mathrm{C}$ for 30 hours in vacuum (68). are depicted in Figures 32 and 33. Analogous to the findings of Fraser et al (69), Figure 33 shows the interaction of these ledges with surfacedislocation sites.

Screw dislocations acting as pit sources at grain boundaries have also been observed by Votava $(70)$ in the evaporation of $\mathrm{NaCl}$ and by Berghezan ${ }^{(71)}$, who noted screw spirals only at twin boundaries in the thermal etching of copper.

* This photomierograph was taken by Dr. H. W. Paxton of the Metal Iurgy Department, Carnegie Institute of Technology. 


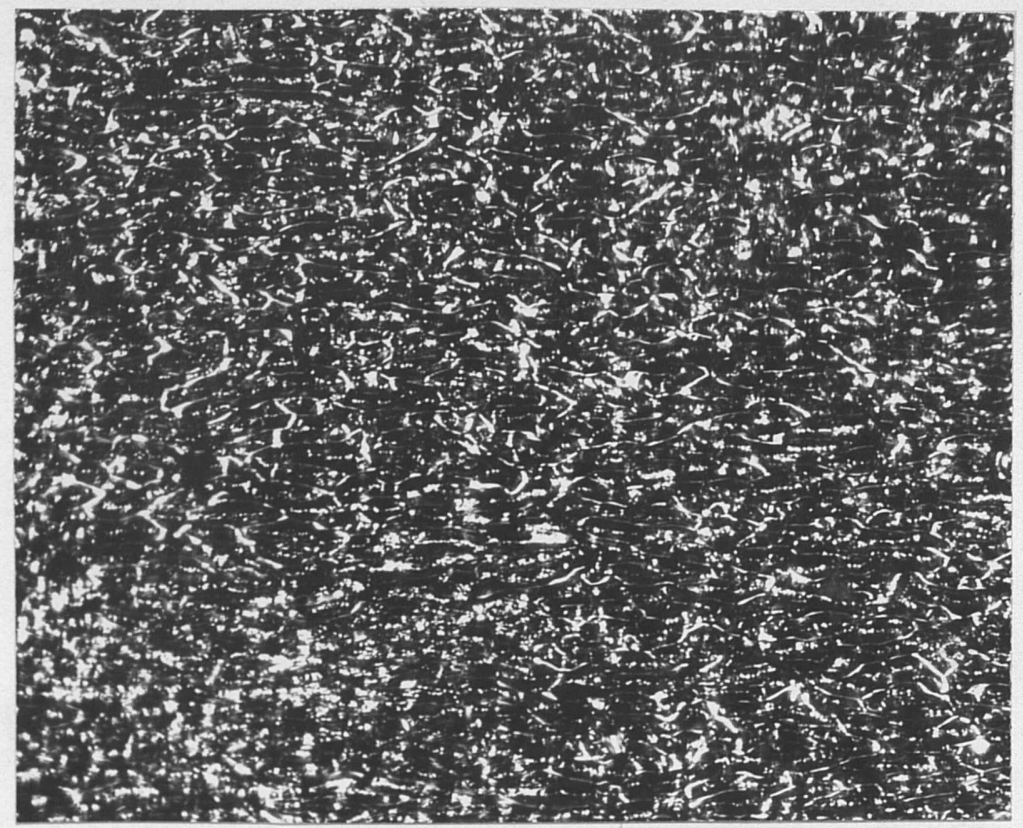

Figure 27. $\{110\}$ surface after evaporation at $744^{\circ} \mathrm{C}$ for 700 min., cylinder single erystal, 200x, 〈110〉 horizontal.

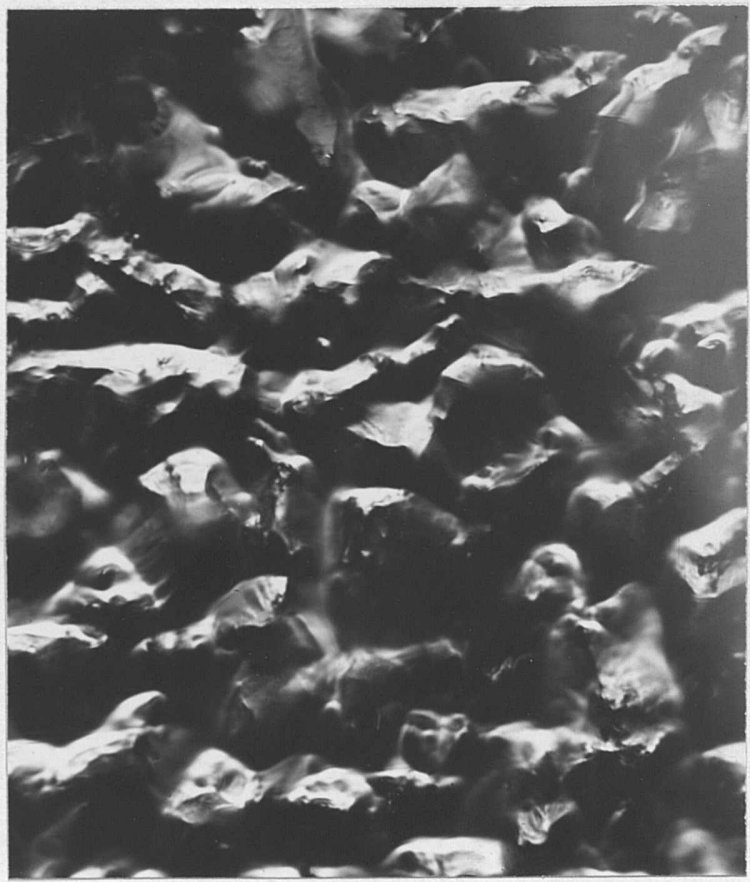

Figure 28. $\{110\}$ surface after evaporation at $744^{\circ} \mathrm{C}$ for 700 min., cylinder single crystal, 1000x. 


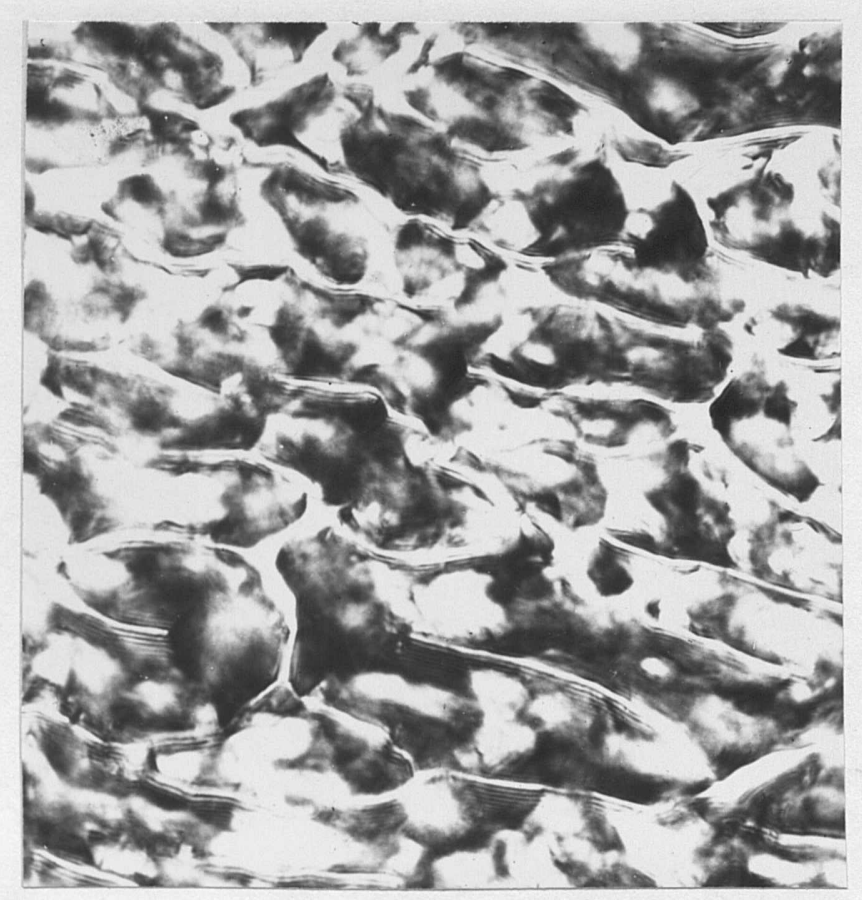

Fỉgure 29. $\{110\}$ surface after evaporation at $744^{\circ} \mathrm{C}$ for $700 \mathrm{~min}$., cylinder single crystal, oblique lighting, 1000X.

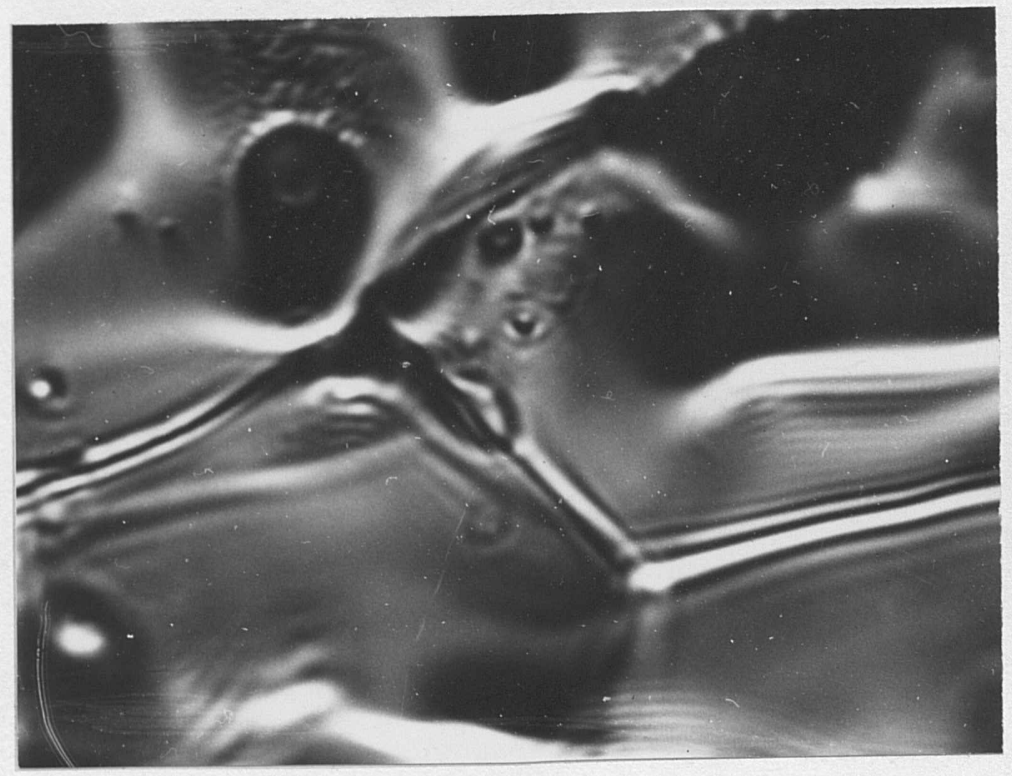

Figure 30. Grain boundary of polyerystal after evaporation at $727^{\circ} \mathrm{C}$ for 528 min. $1000 \mathrm{X}$ blown up $5 \mathrm{X}$ for reproduction. 


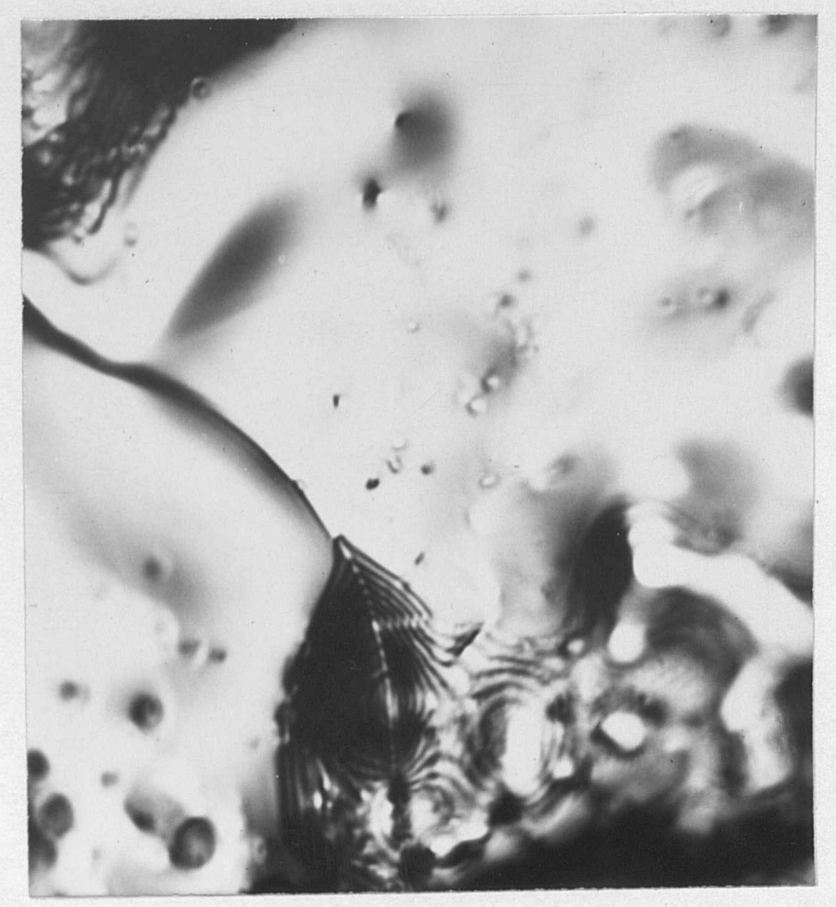

$-63-$

Figure 31. Grain boundary of polycrystal evaporated at $727^{\circ} \mathrm{C}$ for 528 min., 1000X.

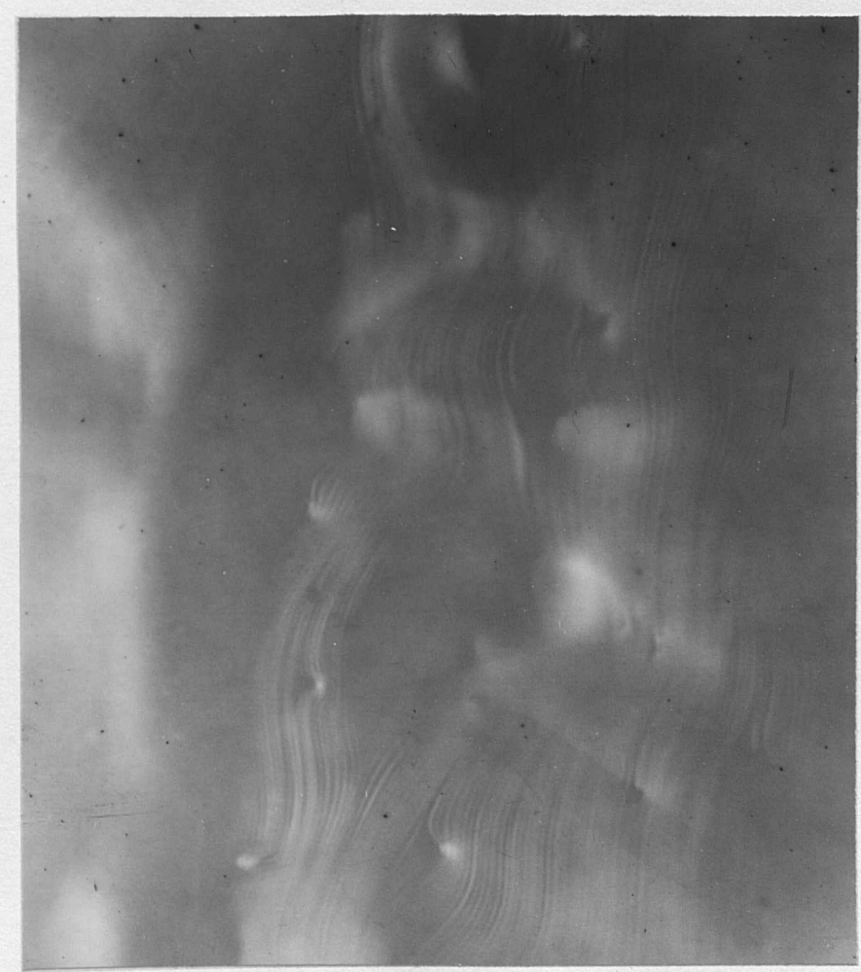

Figure 32. Ledges parallel to grain boundary of chromium polycrystal, grain boundary at left, $3000 x$. 


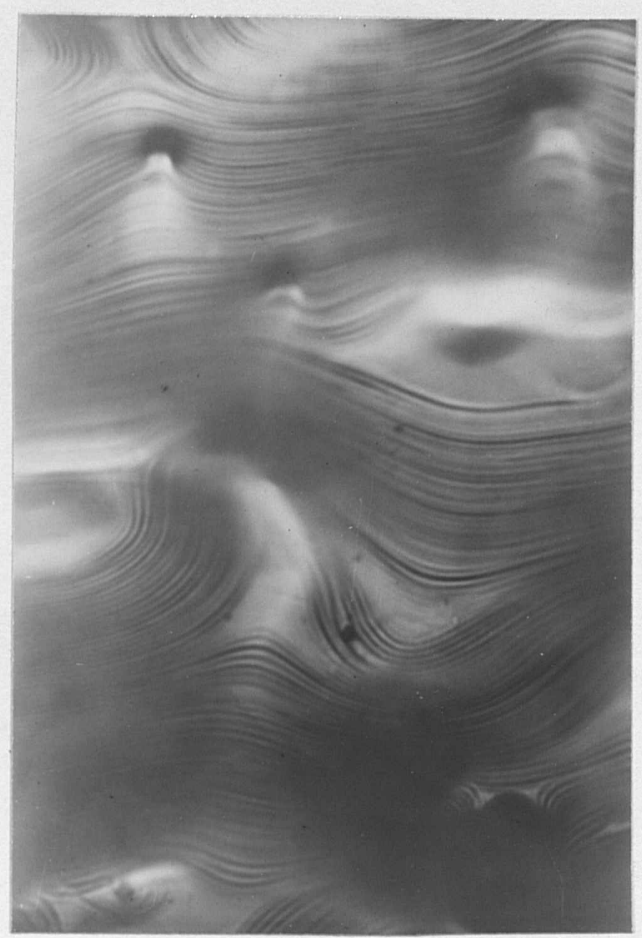

Figure 33. Ledges on chromium polycrystal, 3000X.

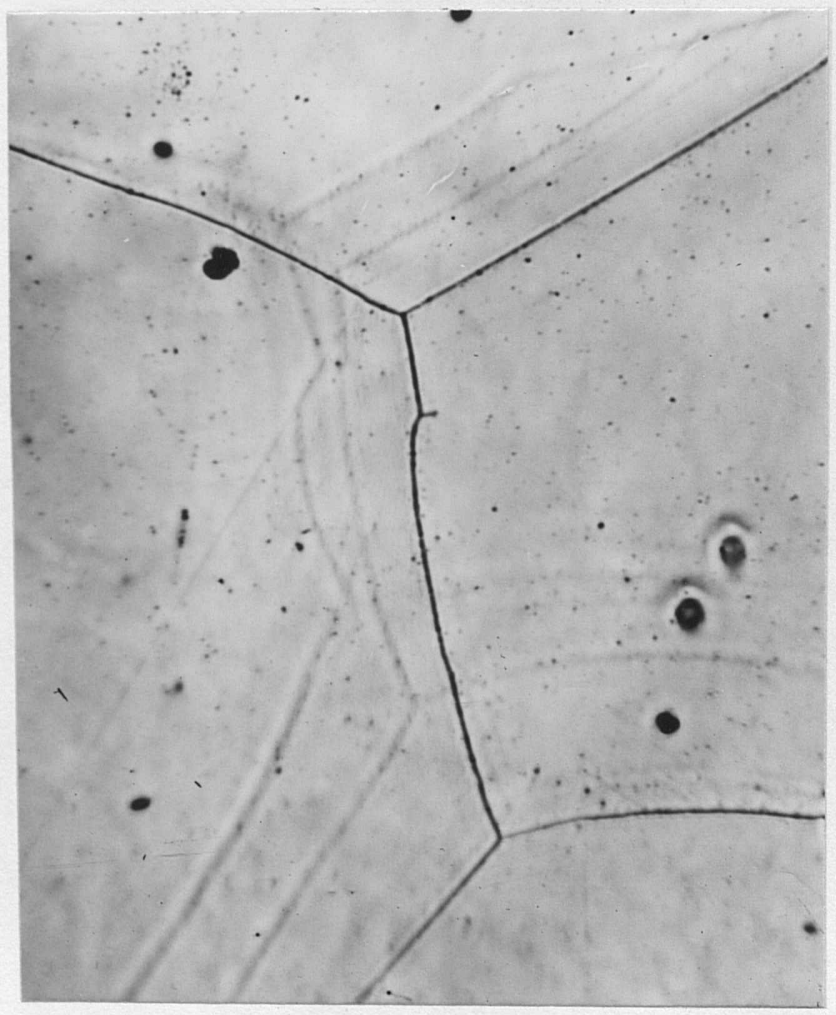

Figure 34. Polycrystalline cylinder, annealed in argon at $800^{\circ} \mathrm{C}$ for two hours, 50X. 


\section{f. Crystal Edges as a Source of Monatomic Ledges}

The action of crystal edges as ledge sources requires the study of crystals with a higher degree of perfection than was the case in the present work. However, evidence of this nature is provided by two recent experiments on non-metallic crystals. Recent work by Sears on p-toluidine single crystals, in which he studied evaporation under a small jet of air that was undersaturated with p-toluidine, showed that evaporation proceeds readily at the crystal edges at very slight undersaturations. On the other hand, when the small jet was directed on the center of a crystal face, an undersaturation of $60 \%$ (air at $40 \%$ of saturation) was required to produce evaporation. Assuming a reasonable value for the crystal-vapor interfacial free energy, it may be calculated from nucleation theory that this undersaturation is critical for appreciable rate of formation of monatomic, disc-shaped holes in the crystal surface.

Due primarily to the high crystal-vapor interfacial free energy of metals, a considerably higher critical undersaturation wo uld be expected for nucleation of holes in a metal surface. In fact, the critical undersaturation is estimated from nucleation theory to be more than $90 \%$ (less than $10 \%$ of saturation). Now it may be shown from equation (20) that for a monatomic ledge spacing of $\lambda_{0}$ at $p=0$,

$$
\begin{aligned}
n(s)_{\min } & =n(s)_{e} \operatorname{sech}\left(Q \lambda_{0} / 2\right) \\
& =0.1 \mathrm{n}(\mathrm{s})_{e} .
\end{aligned}
$$

corresponding to a maximum undersaturation of $90 \%$. Therefore, nucleation of holes in perfect metal crystal surfaces would not be expected. 
Newkirk ${ }^{(73)}$ studied the evaporation of $\mathrm{HgI}_{2}$ and observed that macroscopic ledges (several microns in height) appeared near certain crystal edges and grain boundaries and proceeded to traverse the crystal suriace. It is believed that these polyatomic ledges arose from coalescence of monatomic ledges which originated at the crystal edge. The role of these macroscopic ledges in the vaporization process will be discussed in a later section.

\section{g. Effects of Pores and Cracks}

It is evident that these will behave simply as crystal edges, thus, in effect, decreasing the diameter of the crystal.

\section{h. Surface Pitting of Polycrystals}

Prior to evaporating polycrystalline silver cylinder specimens, the crystals generally were annealed under a silver cover in an alundum boat in purified argon at ambient pressure and $800^{\circ} \mathrm{C}$ for two hours in order to stabilize the grain size. Ghosts of the grain boundaries were observed, indicating that the boundaries were moving under capillary driving forces in an a.lternate grooving and breaking away mechanism as proposed by Mullins (74). This phenomenon is illustrated in Figures 34 and 35 where the frequently observed case of an apparent twin boundary becoming discontinuous and then stopping within a grain is also shown. Following longer periods of evaporation at $744^{\circ} \mathrm{C}$, surface structures were obtained which could be correlated with those obtained on single crystals. Typical photomicrographs are presented in Figures 36 and 37. Figure 36 shows a grain with a surface orientation close to $\{111\}$ at the center of the figure. Figure 37 shows a grain with $\sim\{111\}$ surface at left and $\sim\{110\}$ surface at the right. 


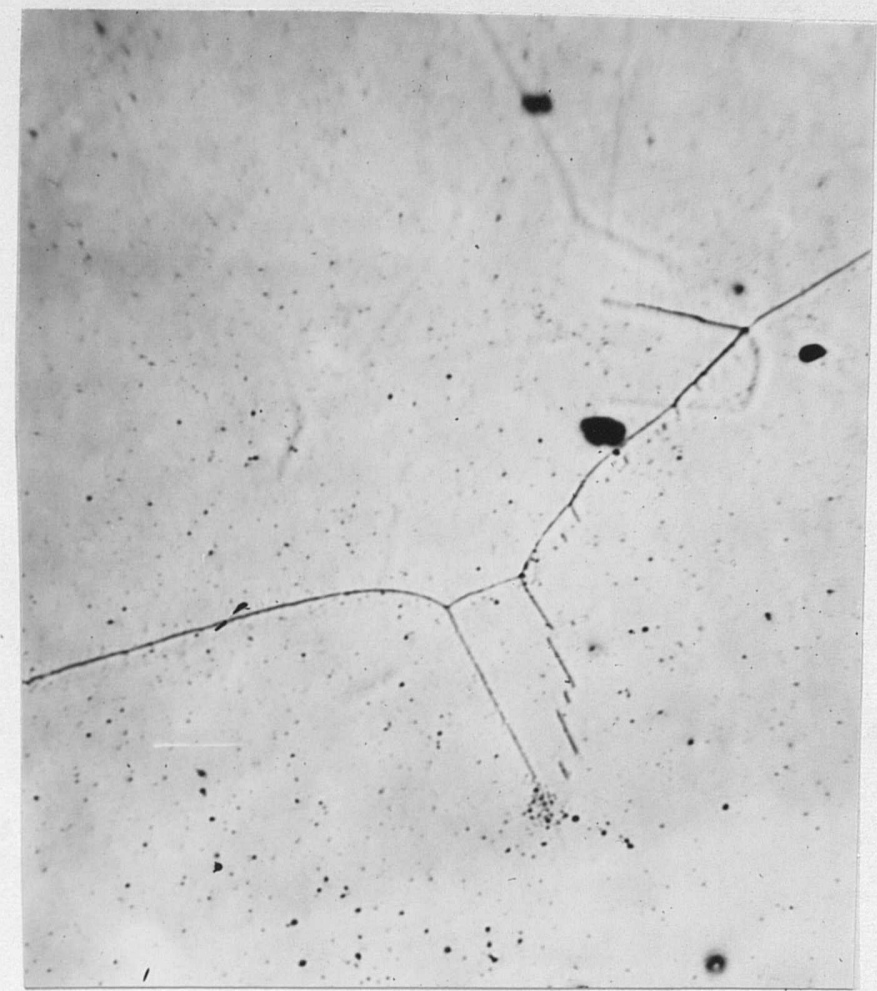

Figure 35. Polycrystalline cylinder, annealed in argon at $800^{\circ} \mathrm{C}$ for two hours, 50x.

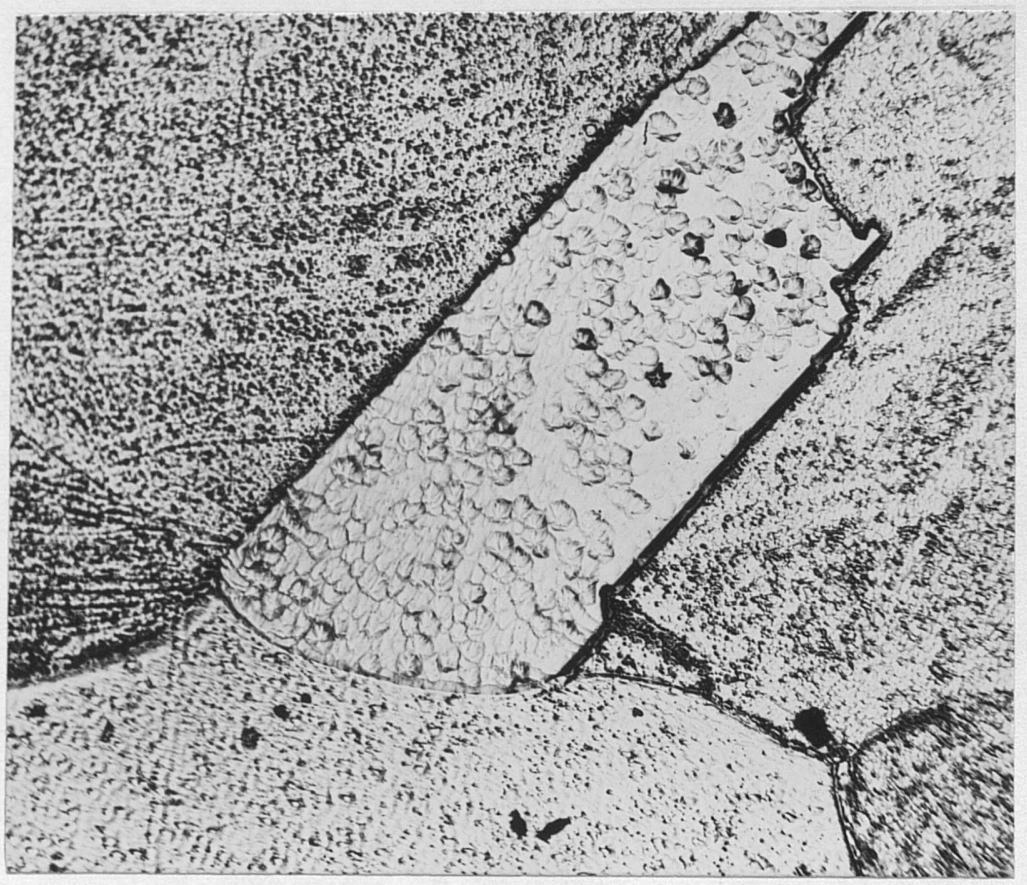

Figure 36. Polycrystalline cylinder ${ }^{\text {evaporated at } 6950}$ min., 200x. 


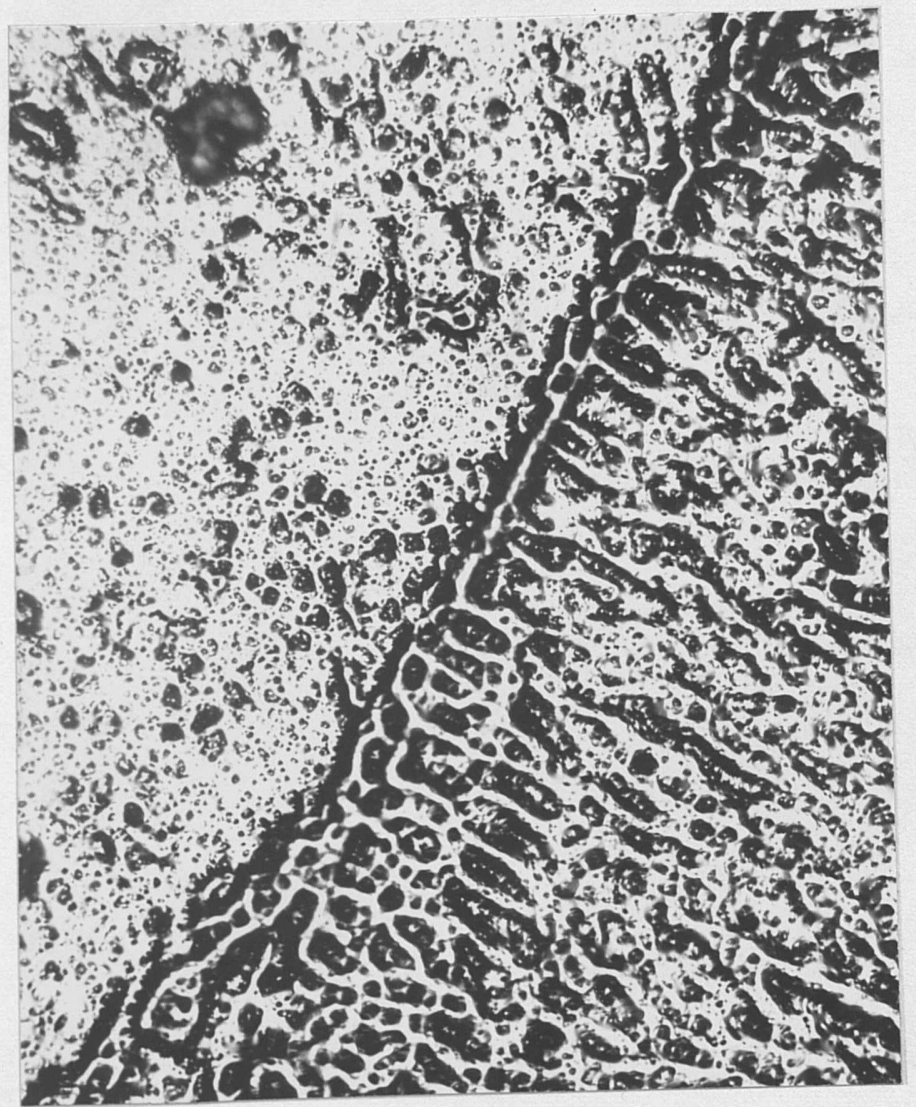

Figure 37. Polycrystalline cylinder, evaporated at $744^{\circ} \mathrm{C}$ for 1306 min. $1000 x$. 


\section{i. Macroscopic Ledges}

The presence of macroscopic ledges on evaporating surfaces has been definitely established experimentally in the present work for silver, see Figure 29, and by others for potassium chloride(25), copper (75), germanium $^{(65)}$, chromium $(68,69)$, and various inorganic compounds, e.g• mercuric iodide(73). Nevertheless, none of the explanations that have been advanced to explain such ledges have general applicability. Polytypism has been advanced as a possible explanation of macroscopic steps in cases where the step source lies within the crystal such as a dislocation source ${ }^{(76)}$. This postulation seems reasonable in cases where the observed step heights are of the order of one or two monomolecular steps as is the case with silicon carbide $(77)$ and other inorganic compounds. However, in cases where the macroscopic step is of the order of 50 to 1000 monomolecular steps ${ }^{(78)}$, the polytypism argunent probably no longer holds.

Dawson and Watson ${ }^{(79)}$ have proposed that molecular size leads to multilayer steps in the case of n-pentacontanol-1. This proposal would apply only to the case of molecular crystals and would not explain steps on crystals in which the evaporation or diffusion element is an atom as is the case with most metals.

Lang $(80)$ made the very interesting proposal that spirals and macroscopic steps arise from growth accidents at cap-shaped roid nuclei on the surface or at other internal defects. Again, this argument applies only to ledges from internal ledge sources.

It has been noted above that crystal edges have been demonstrated. to be ledge sources. Further, the present work and other observations (42) 
indicate that the macroscopic steps lie along close packed directions. In addition, the present work shows that high index surfaces tend to break up into low index facets during evaporation. This indicates that surface free energy controls the formation of macroscopic steps. Near a ledge source where the step spacing increases from two $\AA$ up to several hundred $\stackrel{A}{A}$, the surface will pass through various low index orientations. When these orientations are favorable, a step of low index surface can form with the movement of very few atoms.

Altermatively it has been suggested that macroscopic steps arise due to the presence of groups of imperfections and/or impurities on certain portions of the crystal surface ${ }^{(73)}$. These regions would have the effect of slowing down a monatomic ledge so that it is overtaken by monatomic ledges following it, these ledges then coalescing to form a polyatomic ledge.

In either case, it is formally clear that such a ledge, once formed, would proceed to traverse the crystal surface at a velocity that is low with respect to the velocity of a monatomic ledge. Also it would serve as a source for monatomic ledges and, in cases where the distance between these macroscopic ledges was not great relative to the width $x_{0}$ of the perturbed strip at a source of ledges, the presence of macroscopic ledges would cause positive deviations from the limiting law, equation (30).

2. Evaporation Rate Measurement

a. Continuous Evaporation Rate Measurement

It was found that the continuous weight measurement of the silver target was of little more than qualitative use due to the errors cited in Part II-B-5. However, as is shown for a typical case in Figure 38 , 


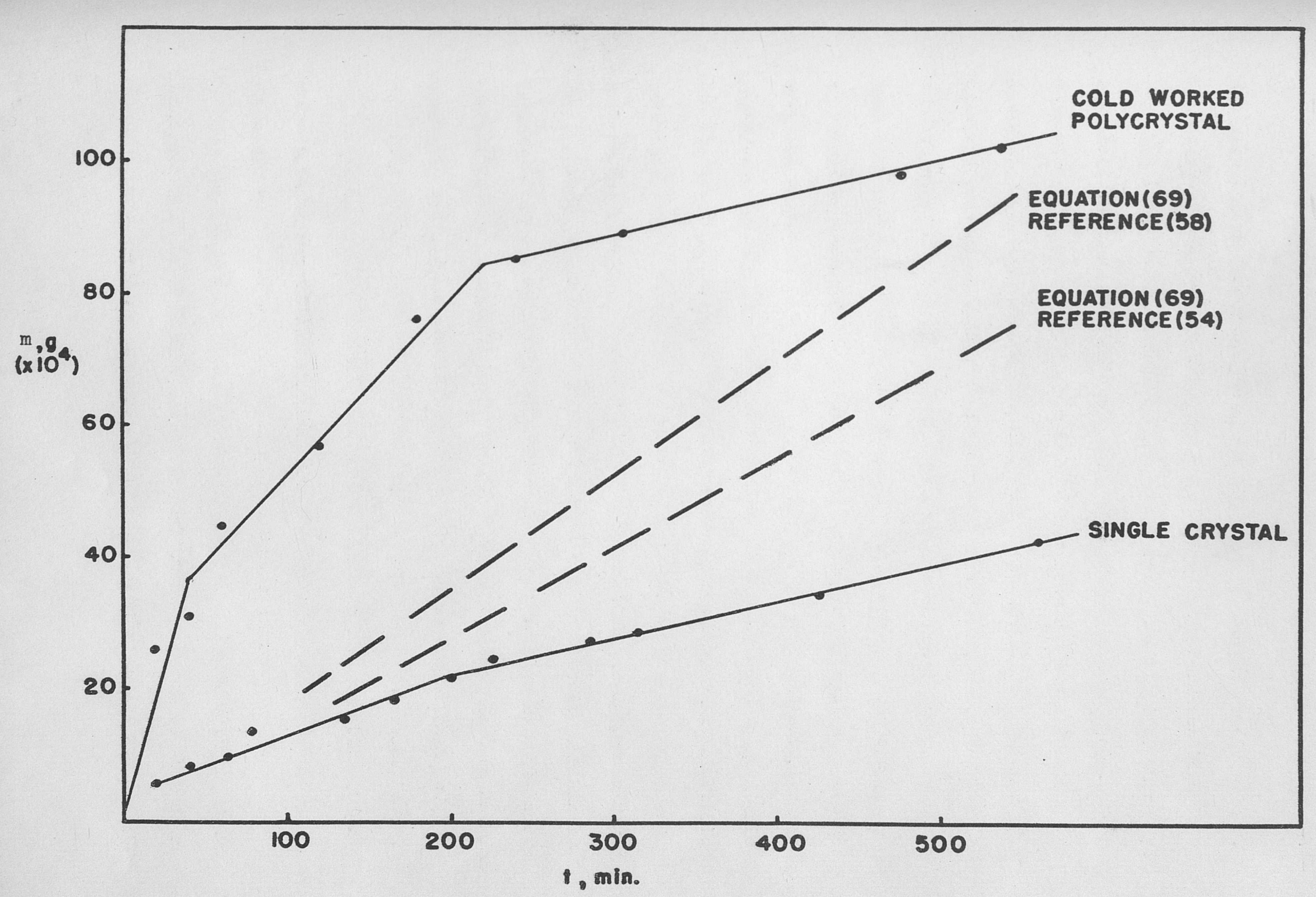

Figure 38. Evaporation Rates of Spherical Crystals at $727^{\circ} \mathrm{C}$. and Rates Calculated from the Equilibrium Pressure. 
the measurement did show that the evaporation rates from annealed polycrystals, from single crystals, and from worked polycrystals all approached a linear rate after an incubation period of about an hour except in the case of worked crystals where this period was somewhat longer. As a result, all crystals were evaporated for one hour and removed from the apparatus and weighed before undertaking an average eva.poration rate experimental determination.

In addition, the results did corroborate the finding that $\alpha$ was less than unity. Although the error is probably large, the results shown. in Figure 42 yield $\alpha \cong .33$ and .41 in comparing the limiting rates with the equilibrium pressures determined by McCabe and Birchenall (58) and Kornev and Vintaikin ${ }^{(54)}$ respectively.

In this and in the following section, the measured evaporation rates are compared with both of the above equilibrium determinations. Most of the earlier determinations of the equilibrium pressure of silver are probably in error due to the reasons reviewed by Birchenall and Schadel ${ }^{(81)}$. McCabe and Birchenall, using the Knudsen cell technique, controlled and measured the temperature very accurately, as well as determining the correct orifice size for equilibrium data. Their results,

$$
\log p=\frac{-65,460}{2.303 R T}+9.003
$$

where $p$ is in mm. of mercury, are reported for these above-mentioned reasons.

On the other hand, Kornev and Vintaikin, used a mass spectrometer together with a radioactive tracer technique in measuring the vapor pressure, 
techniques which could be more accurate than the Knudsen cell weight loss method, since they can be utilized at lower temperatures, giving rise to a broader temperature range to fit a curve to the experimental data. However, these authors are silent about their technique of temperature control. Therefore both equilibrium measurements are reported. These latter authors obtained,

$$
\log p=\frac{-68,100}{2.303 R T}-8.465
$$

At any rate, as can be seen in the results for $\alpha$, both measurements are in fair agreement.

\section{b. Average Evaporation Rate Determination}

(1) Experimental Results

As shown in Table IV, the results for $\alpha$ at $744^{\circ} \mathrm{C}$ for the close packed planes agree fairly well with the predicted value of $1 / 3$. The higher value for $\{110\}$ is in accordance with the observation that this surface tended to break up into close packed plane facets. Thus the $\{110\}$ evaporation rate will be that from $\{111\}$ type planes but with a higher true evaporating area than the apparent area of the $\{110\}$ surface due to the inclination of the $\{111\}$ facets to the $\{110\}$ surface. The result that the large grain polycrystal has an evaporation coefficient intermediate to $\{111\}$ and $\{110\}$ is consistent with this reasoning since the surface orientations vary from $\{111\}$ to $\{110\}$ as shown in Figure 37 . In addition, since the grain size was so large, it is expected that the perturbation due to the grain boundaries is small.

The higher evaporation rates at $843^{\circ} \mathrm{C}$ can be due to two circumstances. Firstly, it is impractical to make a run of longer than about two hours at 
Table IV.

Calculation of $\alpha$ for Various Crystal Planes and Temperatures

\begin{tabular}{|c|c|c|c|c|}
\hline \multirow{2}{*}{$\begin{array}{c}\text { Crystal } \\
\text { Plane } \\
\end{array}$} & \multirow{2}{*}{$\begin{array}{c}\text { Temp. } \\
\text { o } \\
\end{array}$} & \multirow{2}{*}{$\mathrm{g} \cdot / \mathrm{cm}_{0}{ }^{\mathrm{J}} \mathrm{sec}_{0}$} & \multicolumn{2}{|r|}{$\alpha$} \\
\hline & & & Ref. (58) & Ref. $(54)$ \\
\hline$\{100\}^{*}$ & 101.7 & $3.53 \times 10^{-8}$ & $0.28 \pm 0.03$ & $0.36 \pm 0.04$ \\
\hline$\{111\}^{*}$ & 1017 & $3.80 \times 10^{-8}$ & $0.31 \pm 0.03$ & $0.39 \pm 0.04$ \\
\hline$[110)^{*}$ & 1017 & $5.42 \times 10^{-8}$ & $0.44 \pm 0.04$ & $0.56 \pm 0.06$ \\
\hline $\begin{array}{c}\text { Polycrystal*, } \\
\text { grain diameter } \\
5 \text { ma. }\end{array}$ & 1017 & $4.28 \times 10^{-8}$ & $0.35 \pm 0.04$ & $0.44 \pm 0.04$ \\
\hline$\{110\}^{*} \neq$ & 1116 & $1.37 \times 10^{-6}$ & $0.62 \pm 0.06$ & $0.69 \pm 0.07$ \\
\hline $\begin{array}{c}\text { Polycrysta. } \\
\text { grain diameter } \\
5 \mathrm{~mm} .\end{array}$ & 1116 & $1.21 \times 10^{-6}$ & $0.54 \pm 0.06$ & $0.61 \pm 0.06$ \\
\hline $\begin{array}{c}\text { Polycrystal } \\
\text { grain diameter } \\
1 \text { mm. }\end{array}$ & 1116 & $1.45 \times 10^{-6}$ & $0.66 \pm 0.07$ & $0.73 \pm 0.07$ \\
\hline $\begin{array}{c}\text { Polycrystal } \\
\text { cold worked }\end{array}$ & 1116 & $2.64 \times 10^{-6}$ & $1.33 \pm 0.13$ & $1.20 \pm 0.12$ \\
\hline
\end{tabular}

Equilibrium Pressure

\begin{tabular}{|c|c|c|c|}
\hline & $\underline{\mathrm{T}},{ }^{\circ} \mathrm{K}$ & $\mathrm{J}_{2} \mathrm{~g} \cdot / \mathrm{cm}_{0}{ }^{2}$ sece & $p_{2} m_{0} m_{0}$ \\
\hline Ref. (58) & 1017 & $1.24 \times 10^{-7}$ & $0.832 \times 10^{-5}$ \\
\hline Ref. (54) & 1017 & $0.974 \times 10^{-7}$ & $0.661 \times 10^{-5}$ \\
\hline $\operatorname{Ref} \cdot(58)$ & 1116 & $2.20 \times 10^{-6}$ & $1.55 \times 10^{-4}$ \\
\hline Ref. (54) & 1116 & $1.97 \times 10^{-6}$ & $1.38 \times 10^{-4}$ \\
\hline
\end{tabular}

* Average of three runs of $12 \mathrm{hrs}$. at $1017^{\circ} \mathrm{K}$ or $2 \mathrm{hrs}$. at $1116^{\circ} \mathrm{K}$.

+ Average of two rums of $12 \mathrm{hrs}$. at $1017^{\circ} \mathrm{K}$ or $2 \mathrm{hrs}$. at $1116^{\circ} \mathrm{K}$.

F One run of $12 \mathrm{hrs}$. at $1017^{\circ} \mathrm{K}$ or $2 \mathrm{hrs}$.at $1116^{\circ} \mathrm{K}$. 
this temperature since the evaporation rate is so high. Therefore, since, as discussed in the preceding section, there is some uncertainty as to whether the evaporation rate is linear in the first hour, the results for $\alpha$ at high temperatures may be high. The $744^{\circ} \mathrm{C}$ results are less sensitive to such a possibility. Secondly, Frank $(27,43)$ has shown that all surfece planes, with the exception of the low index planes present in the Gibbs-Wulff shape for face centered cubic materials, are subject to "surface melting" or melting of second order bonds at temperatures approaching the melting point. This would cause the increase in $\alpha$ a.s found experimentally at the higher temperature.

In agreement with the results shown in Figure 38 , it was found that a cold worked specimen had an average evaporation rate much higher than amealed specimens due to the high initial rate of evaporation. This is due to the instability of surface imperfections during this period. The grain boundaries are mobile, moving during recrystallization and grain growth. In addition, dislocations are also mobile, as shown by Gilman (82), decreasing in number from a typical value for a cold worked material of $10^{12}$ per $\mathrm{cm}^{2}$ to $\sim 10^{8}$ per $\mathrm{cm}^{2}$ for an annealed material. Thus at each successive position of intersection of dislocations or grain boundaries, ovaporation at an enhanced rate will take place both due to the perturbation discussed in Part $I-C$ and due to the increased free energy at such sites due to strain energy. Once the structure has stabilized, the evaporation again appears to follow the usual kinetics as shown in Figure 38.

In general, it was very difficult to form a stable fine grained. sample due to the purity of the silver used in this work. However, one sample of a relatively finer grain size was produced, though this grain 
size was still fairly large. The results indicate that the finer grain size specimen evaporates at a higher rate as predicted. This finding is equivocal, however, the spread between the large and small grain size results lying within the experimental error. Also, a fluctuation in orientation of the polycrystalline grains could contribute to such behavior.

The absolute accuracy of these results is, of course, also subject to the deviation of the equilibrium vapor pressure measurements used for comparis on $(54,58)$.

Finally, the results presented in Table IV are consistent with the predicted perturbation noted in Table III. For $\mathrm{T} \equiv 1017^{\circ} \mathrm{K}, r_{\mathrm{a}}=1.47$ $\times 10^{-5} \mathrm{~cm} \cdot, A_{a}=0.68 \times 10^{-3}$ and $A_{0}=0.21$ for a number of pit sources $=$ $10^{6}$, the number observed experimentally. Thus the number of imperfections in the surface is not sufficient to appreciably affect the limiting value of $\alpha \cong 1 / 3$.

(2) Other Research

Of the numerous data available in the literature on metal evaporation rates only a few may be compared with the predictions of the present theory due to limitations of precision measure and lack of control of experimental variables. Probably the best values of $\alpha$, the vaporization coefficient in equation (2), are for $p=0$ and were obtained by comparing the results of Knudsen and Langmuir measurements of vapor pressure, i॰e. $\alpha_{p=0}=p_{e}($ Langmuir $) / p_{e}($ Knudsen). In general, experimental vapor pressure data from either method, plotted as In $p$ versus 1/I, have a standard deviation of about 10\%. Also, the difference between the results of separate investigations is sometimes as high as 
80\%. Therefore, many of the values of $\alpha$ determined in this manner at $p=0$ might easily be obscured by experimental error. Further, the physical state of the test specimens is usually incompletely specified. The metal specimens were usually polycrystal line, and the grain size is generally not given. Also, not enough morphological information is given to permit assessment of the concentration of surface imperfections on the grains, such as pores, cracks, screw dislocations, macroscopic ledges, etc.

A few data relating to the effect of decrease of crystal or grain. size of the metal on increasing $\alpha$ are found in the literature, although it is emphasized that orientation fluctuation or dislocation. concentration, terms usually unspecified, could account for the observed results. Rosenhain and Bwen ${ }^{(33)}$ found that the rates of evaporation of polycrystalline silver, copper, and zinc increase appreciably with decrease in grain size. The evaporation rate of silver increases by a factor of 1.5 upon decreasing the grain diameter from about $1 \mathrm{~cm}$ to $0.01 \mathrm{~cm}$. Similarly, Fonda $(34)$ observed that the evaporation rate of polycrystalline tungsten having a grain size of about $0.01 \mathrm{~cm}$ is $40 \%$ higher than for specimens having a grain size of about $0.1 \mathrm{~cm}$. Inasmuch as it is reasonable to presume that the Gibbs- Thomson effect is not contributing appreciably to these phenomena, one may consider the results to be in qualitative support of the theoretical prediction.

As initially noted by Harteck ${ }^{(16)}$ and Eucken ${ }^{(17)}$, the comparison of the vapor pressures of copper and silver determined by the Langmuir method ${ }^{(84)}$ with Knudsen results yielded values for $\alpha$ of 0.1 to 0.3 . Bradley and Volans (85), in a study of evaporation of single crystals of 
potassium chloride, find that $\alpha$ at $p_{e}>p>0$ is 0.72 for $\{100\}$ and $\{111\}$ planes and 0.63 for $\{110\}$ planes. Allen ${ }^{(86)}$ studied various planes of silicon single crystals and found that $\alpha$ was less than one for these planes. In each of these cases, the kinetic treatment given above should apply provided that entropy factors can be neglected. On the other hand, Wessel $(19,87)$, using a direct method, found that $\alpha \geq 0.92$ for silver at $p=0$. Although his technique for determination of $\alpha$ is the only direct method reported, it is subject to possible experimental error in that the Knudsen effusion areas in his torsion device are small with respect to the cylinder surface, which comprises the Langmuir area. Also, the morphology of the cylinder surface is not specified, and the temperature is not precisely known.

Similarly, a method entailing comparison of the evaporation rate from a flat surface with that from a surface with a number of holes drilled in it ${ }^{(18)}$ indicates $\alpha=1$ for beryllium and several other metals. In this method the holes act to an unspecified degree as quasiKnudsen cells. However, it may 2 lso be subject to experimental error, and, again, the morphology of the metals is not specified.

\section{Measurement of $\alpha$ in Argon}

Single crystals and polycrystals, in both cases cylindrical specimens, were heated in ambient pressure of purified argon at $850^{\circ} \mathrm{C}$ for 48 hours. No weight change was detectable indicating that under such conditions $\alpha \leq 1 / 2700$. 


\section{Application of Results}

\section{Wire Filaments}

The results of this research are chiefly of interest in prem senting a mechanism for evaporation. However, as a result of this development, several practical applications of the results are suggested. One such application is in the possible use of single crystal whiskers as filaments for critical applications. If a highly perfect whisker with low index bounding surfaces could be grown, the possibility of localized evaporation leading to hot spots would be reduced and the average evaporation rate reduced with respect to polycrystalline wire specimens. The absence of surface-dislocation intersections would assure an evaporation coefficient approaching 1/3. Further, what evaporation did take place would occur by the recession of ledges from the edges formed by the boundary planes, a circunstance which would lead to the sustenance of a uniform cross-section of the filament.

\section{Catalyst Application}

Since surface catalysts are generally associated with active surface sites, it is expe cted that an increased number of kink positions on a given surface will increase the number of active sites on that surface. Therefore, if a specific metal is to be used as a catalyst with high surface activity, a preliminary treatment of the surface involving evaporation at high temperature, where the ledge spacing $\lambda$ is small, will provide a surface with a large number of ledges and kinks and thus provide a highly active surface. 


\section{Langmuir Vapor Pressure Measurements}

The results of the present investigation indicate that vapor pressure determinations by the Langmuix method can be in error by as much as a factor of three, dependent on the prior history of the surface of the sample being studied. The number of imperfections in the surface, the grain size, the sample purity, and the specimen geometry all affect and thus the apparent vapor pressure determined by the Langmuir method. If Langmuir experiments are carried out on flat, low index, single crystal surfaces of high perfection, the vapor pressure can be determined by using the factor $\alpha=1 / 3$ in equation (2).

\section{Other Kinetic Processes}

Other phase change reactions involving a rate controlling step other then heat transfer could be described by an analogous kinetic treatment. Processes suitable for such an analysis include dissolution and oxidation. 


\section{PROPOSED EXXERIMENTS}

\section{A. Evaporation Rate Measurements}

\section{Perfect Crystals}

Based on the encouraging results of the experiments discussed in the preceding section, several experiments can be suggested that have a high probability of yielding positive results. The evaporation rate measurement from single crystals of germanium or silicon with a low concentration of dislocations, say < 10 per $\mathrm{cm}^{2}$, crystals which have been produced recently on a development basis, would provide a critical test of the hypothesis that ledges emanating from surface-dislocations approach the same spacing as those emanating from a crystal edge. That this is indeed the case is indicated by the fact that $\alpha$, for crystals of silver with $10^{6}$ evaporation sites per $\mathrm{cm}^{2}$, was near the value predicted by equation (30). Further, a comparis on of the evaporation rate from such a high purity crystal could be compared with the rate from crystals of lower degrees of perfection, $10^{4}$ to $10^{8}$ dislocations per $\mathrm{cm}^{2}$. The frectors $\mathrm{A}(\boldsymbol{\lambda})$ and $B(\lambda)$ discussed on page 31 could then be explicitly determined as a function of degree of surface perfection.

Other possible sources for nearly perfect crystals are whiskers and/or polyhedra grown from the vapor phase using the techniques discussed by Sears ${ }^{(88,89)}$. Frank ${ }^{(29)}$, Volmer ${ }^{(15)}$, Newkirk ${ }^{(73)}$, and others have reported high perfection metal and non-metal crystals grown by such techniques.

2. Evaporation Under Varying Undersaturation

It is predicted on page 35 that $\alpha$ is a linear function of undersaturation, varying from $1 / 3$ at infinite undersaturation to 1 at zero 
undersaturation. The limiting values have been confirmed. The dependence of $\alpha$ on undersaturation could be determined by arranging a Knudsen cell so that a beam of atoms could be impinged on an evaporating sample in an apparatus similar to that shown in Figure 11. By controlling the temperature of the Knudsen cell and the sample, evaporation under varying underm saturation, as well as condensation under varying supersaturation, could be studied.

\section{Transient Evaporation Rates}

As discussed in Section II, the initial evaporation rate appeared to decrease from a high rate to a limiting rate in about an hour. The microspring balance was not sensitive enough to follow this transient period quantitatively. An apparatus in which a radioactive tracer in the sample would enable the detection of the rate of deposition on the target by a radiation counting method would greatly increase the sensitivity of the measurement and enable the transient kinetics to be determined. Equations (69) and (70) wo uld still hold for this case. As an example of the enhanced sensitivity consider that to detect a weight gain by the microspring deflection requires the deposition of $9 \times 10^{-5} \mathrm{~g}$. on a $3 \mathrm{~cm}$. diameter target or about $10^{16}$ atoms per $\mathrm{cm}^{2}$. If a tracer of 1 part in $10^{6}$ was added to the sample and a series of depositions for fixed time periods made, the same deposition would give rise to $10^{10}$ active atoms which could be fitted to a radioactive decay law to determine the amount deposited as a function of time and thus to determine the transient evaporation kinetics. 


\section{B. Surface Morphology}

\section{Perfoct Crystals}

As an adjunct to Part III-A-I, the surface of the high perfection crystals could be studied by means of phase contract micrography and ledge "decoration" techniques to determine the interaction of edge nucleated ledges and dislocation nucleated ledges.

\section{Polycrystals}

In the course of the present investigation, some peculiarly etching surface structures were observed on polycrystals evaporated for short periods of time, or in essence "thermally etched." These structures, suggesting twins, are shown in Figures 39 and 40. Studied with an X-ray microbeam and by interferometric techniques, such structures could reveal information regarding the mobility and energy of twin boundaries.

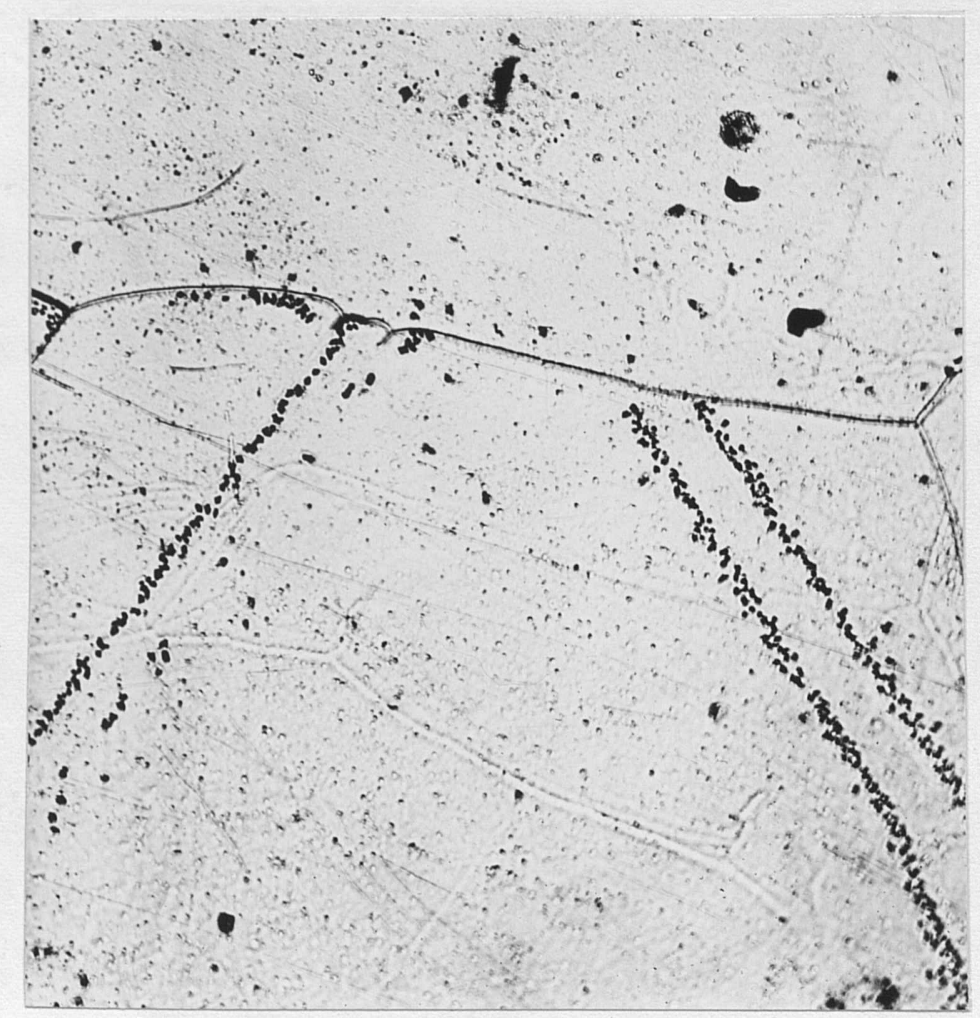

Figure 39. Polycrystalline cylinder, evaporated at $6230^{\circ} \mathrm{C}$ for one hour, 100x. 


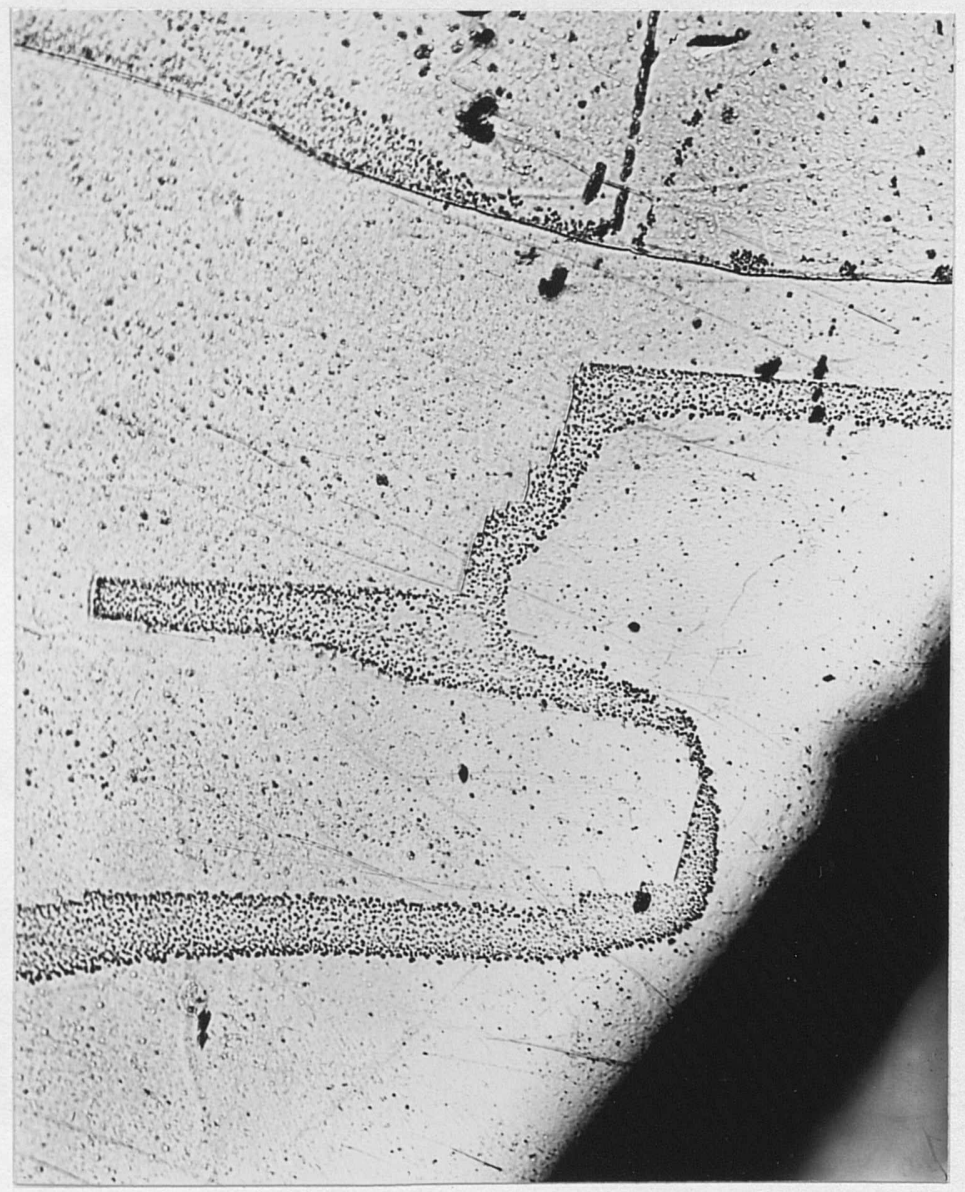

Figure 40. Polyerystalline cylinder, evaporated at $623^{\circ} \mathrm{C}$ for one hour, 100x. 
IV. SUMMARY

\section{A. Theory}

1. The model of a crystal surfa ce in which monatomic ledges are present and the mechanism of evaporation deduced therefrom lead to rate equations that are consistent with experimental observations. The mechanism involves dissociation of atoms from the monatomic ledges, surface diffusion, and deadsorption. The key to this treatment lies in the dynamics of the monatomic ledges.

2. The limiting expression for the evaporation coefficient defined in equation (2) is $\alpha=2 / 3\left(p / p_{e}\right)+1 / 3$. This should apply to the evaporation of large metal crystals that are relatively free of surface imperfections.

3. Positive deviations from the limiting expression would be expected for crystal (or grain) sizes of the order of 1 to 100 microns or less. This deviation is not due to the Gibbs-Thomson effect.

4. Crystal edges should serve as a ready source for monatomic ledges during e vaporation.

5. Macroscopic ledges and screw dislocations should cause positive deviations from the limiting expression for $\alpha$ only if they are present in high concentrations.

6. The expression for the evaporation coefficient of imperfect crystals is $\alpha=\bar{J} / J_{c}$, where $J_{c}$ is given by equation (I) and 
$\bar{J} \equiv \bar{J}_{v}+C_{2} p$ in which $\bar{J}_{v}$ is given by equation (37). In this application of equation (37), $\mathrm{X}^{\prime}$ is interpreted as the half distance between ledge sources such as pores, cracks, macroscopic ledges, etc.

7. In evaporation of metal crystals at large undersaturations, the terminal spacing of monatomic ledges emanating from screw dislocations should equal the terminal spacing of ledges emanating from crystal edges.

8. Considering the area of a perturbed region about a screw dism location spiral at large undersaturations, such that $\lambda<\lambda_{0}$ in this region and the evaporation coefficient is greater than that given by the limiting law, to gether with the surface concentration of screw dislocations, a criterion is found for applicability of the limiting Iaw in terms of the unperturbed fraction $\left(1-A_{0}\right)$ of the surface. From Table III it is seen. that, for a reasonable low concentration of screw dislocations, $10^{5}$ per $\mathrm{cm}^{2}$, and higher temperat ures, the fraction of perturbed area is sufficiently small that the limiting law applies. On the other hand, higher concentrations of screw dislocations and lower temperatures lead to large deviations from the limiting law. It is proposed that this criterion may explain why measured evaporation coefficients approach the limiting value of $1 / 3$ at zero pressure in some experiments while in others the evaporation coefficient is found to be essentially unity. 
9. At small undersaturations such that $19 \rho_{c}>\lambda_{0}$, the limiting law should apply regardless of temperature and concentration of screw dislocations.

10. The present considerations also provide a criterion for the dem gree of undersaturation which will lead to pitting at screw dislocations. At undersaturations sufficiently large that $19 \rho_{c}<\lambda_{0}$, and hence true steady state evaporation from screw dislocations cannot occur, the flux from the region near the screw dislocation will be higher than elsewhere on the surface and pronounced pitting will occur.

\section{B. Experiment}

1. Confirmation of the limiting expression, $\alpha=2 / 3\left(p / p_{e}\right)+1 / 3$, has been obtained in the case of evaporation from low index planes of silver single crystals.

2. It has been found for silver that non-close packed planes break up into low index plane facets, resulting in a higher apparent evaporation rate for the high index planes.

3. The perturbing effect of screw dislocations is consistent with the perturbation predicted theoretically, having little effect on the limiting law in the case of silver.

4. In the course of continuous evaporation rate measurenent, it is shown that the rate is linear after about the first hour. The kinetics during the first hour cannot be unequivocally 
determined by the methods applied in this investigation. A method for the study of these initial kinetics is suggested.

5. The approximate orientation of grains on an evaporated polycrystalline surface can be determined from the surface morphology.

6. No one to one correlation between etch pits and dislocation densities on evaporated surfaces was found. On close packed silver surfaces, etch pit densities of $10^{6}$ per $\mathrm{cm}^{2}$ were obtained, in contrast with a dislocation density of $10^{8}$ per $\mathrm{cm}^{2}$. It is suggested that this lack of agreement is due to the nonmuniformity of the Burger's vector component normal to the surface for the various dislocations intersecting the surface.

7. Evaporation from a cold worked surface results in an evaporation rate considerably higher than from an annealed surface due to the motion of grain boundaries and dislocations intersecting the surface in the former case.

8. It is shown that Langmuir vapor pressure measurements can be in error by as much as a factor of three, dependent on the degree of perfection of the evaporating surface. 


\section{BIBLIOGRAPHY}

1. M. Knudsen, Ann. Physik, 29 (1909)179.

2. Io Lengmuir, Physical Review, 2 (1913) 329.

3. H. Hertz, Wied. Ann., 17 (1882) 193.

4. M. Knudsen, Ann. Physik, 47 (1915) 697.

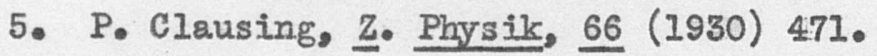

6. J. E. Vance and C. I. Whitman, J. Chem. Phys., 19 (1951) 744.

7. C. I. Whitman, Ibid, 20 (1952) 161.

8. K. Motzfeldt, J. Phys. Chem., 59 (1955) 139.

9. H. Melville, T. Faraday Soc., 32 (1936) 1017.

10. A. W. Searcy and R. D. Freeman, J. Am. Chem. Soc., 76 (1954) 5229.

11. J. D. McKinley, Jr. and J. E. Vance, J. Chem. Phys., 22 (1954) 1120.

12. I. Langmuir, Physical Review, 8 (1916) 149.

13. J. Chirigos, Unpublished research.

14. J. E. Lennard-Jones and A. F. Devonshire, Proc. Roy. Soc., Al56 (1936) 6.

15. M. Volmer and I. Estermann, Z Physik, $\underline{7}$ (1921) I.

16. P. Harteck, Z physik. Chem, 134 (1928) 1.

17. A. Eucken, Metallwirtschaft, 15 (1936) 27,63.

18. R. Speiser and H. L. Johnston, T. Am. Soc. Met., 42 (1950) 283.

19. R. Speiser and J. W. Spretnak, "Vacuum Metallurgy," Electrochemical Society, Boston, 1955 p. 155.

20. M. Polanyi and E. Wigner, Z. physik. Chem., 139A (1928) 439.

21. K. Neumann, Z. physik. Chem., 196 (1950) 16.

22. O. Knacke, I. N. Stranski, and G. Wolff, Z. Electrochem., 56 (1952) 476 . 
23. I. N. Stranski and G. Wolff, Research, 4 (1951) 15.

24. O. Knacke, I. N. Stranski, and G. Wolff, Z. physik. Chem., 198 (1951) 157.

25. O. Knacke and I. N. Stranski, "Progress in Metal Physics," Vol.6, Pergamon Press Ltd., London, 1956, p. 181.

26. L. Brewer and J. S. Kane, J. Phys. Chem., 59 (1955) 105.

27. W. K. Burton, N. Cabrera, and F. C. Frank, Phil. Trans. Roy. Soc., 243A (1951) 299.

28. G. M. Pound, M. T. Simnad, and L.Yang, J. Chem. Phys., 22 (1954) 1215.

29. F. C. Frank and A. J. Forty, Proc. Roy. Soc., 217A (1953) 262.

30. H. Suzuki, J. Phys. Soc. (Japan), 10 (1955) 981.

31. A. J. Forty, Phil. Mag., 43 (1952) 481.

32. A. R. Verma, Ibid, 43 (1952) 441.

33. E. W. Muller, J. App. Phys. 27 (1956) 474.

34. J. K. Trolan, J. P. Barbour, E. E. Martin and W. P. Dyke, Phys. Rev., 100 (1955) 1946.

35. M. Drechsler, Z. Metal1kde, $\underline{47}$ (1956) 305.

36. J. K. Mackenzie, Thesis, University of Bristol, 1950.

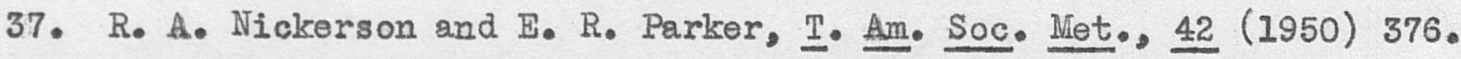

38. L. Onsager, Phys. Rev., 37 (1931) 405.

39. C. J. Smithells, "Metals Reference Book," Vol. II, Interscience Publishers, Inc., New York, 1955, p. 575.

40. F. C. Frank, Disc. Faraday Soc., 5 (1949) 48, 67.

41. N. Cabrera and W. K. Burton, Ibid, 5 (1949) $33,40$.

42. A. R. Verma, "Crystal Growth and Dislocations," Academic Press Inc. New York, (1953).

43. Fo Co Frank, Adv. in Phys., I (1952) 91.

44. N. Cabrera and M. M. Levine, Phil. Mag., I (1956) 450. 
45. F. C. Frank, private communication.

46. G. N. Watson, "A Treatise on the Theory of Bessel Functions," Cambridge University Press, Cambridge (1952).

47. C. A. F. T. Spray, Proc. Phys. Soc., 69 (1956) 689.

48. J. J. Gilman and W. G. Johnston, J. App. Phys., 27 (1956) 1018.

49. R. A. Oriani, J. Chem. Phys., 18 (1950) 575.

50. D. Turnbull, "Thermodynamics in Physical Metallurgy," ASM, Cleveland (1950) 282.

51. R. Gomer and C. S. Smith, "Structure and Properties of Solid Surfaces," University of Chicago Press, Chicago (1953).

52. S. Dushman, "Vacuum Technique," John Wiley and Sons, Inc., New York, 1949 .

53. R. E. Honig and J. Drowart, "ARDC Technical Note 56-555, AD 110374," USAF, Oct. 1956.

54. Y. V. Kormer and E. Z. Vintaikin, Dokl. Akad. Nauk. SSSR, 107 (1956) 661.

55. P. Schissel, J. Chem. Phys., 26 (1957) 1276.

56. W. H. Walker, W. K. Lewis, W. H. MaAdams and E. R. Gilliland, "Principles of Chemical Engineering," McGraw-Hill Book Co., Inc., New York, 1937, p. 152.

57. R. C. Ray and V. Dayal, T. Faraday Soc., 32 (1936) 741.

58. C. L. McCabe and C. E. Birchena11, TAIME, 197 (1953) 707.

59. L. Yang, M. T. Simnad, and G. M. Pound, Acta Met., 2 (1954) 470.

60. P. Evans, Acta Met.. 5 (1957) 342.

61. F. I. Vogel, W. G. Pfann, H. E. Corey, and E. E. Thomas, Phys. Rev., 90 (1953) 489.

62. A. H. A. Meleka, Phil. Mag., I (1956) 802 .

63. W. R. Hibbard, Jr. and C. G. Dunn, Acta Met., 4 (1956) 306.

64. S. G. Ellis, J. App. Phys. 26 (1955) 1140 .

65. A. A. Hendrickson and E. S. Machlin, Acta Met., 3 (1955) 64. 
66. A. Seeger, "Defects in Crystalline Solids," Physical Society, London (1955) 328.

67. W. W. Mullins, J. App. Phys., 28 (1957) 333.

68. H. W. Paxton and E. Passierb, unpublished research.

69. M. J. Fraser, D. Caplan and A. A. Burr, Acta Met., 生 (1956) 186.

70. E. Votava, Z. Metallkde., 47 (1956) 309.

71. Mo Berghezan, unpublished research.

72. G.W. Sears, J. Chem. Phys., 24 (1956) 868.

73. J. Newkirk, Acta Met., 生 (1956) 316.

74. W. W. Mullins, research in progress.

75. F. W. Young, Jr., J. App. Phys., 27 (1956) 554,

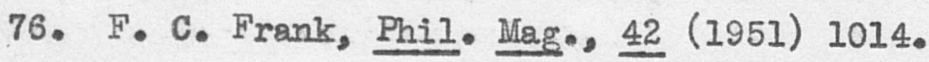

77. A. R. Verma, Ibid, 42 (1951) 1005.

78. S. Amelinckx, C.C. Grosjean, and W. Dekeyser, Ibid, 프 (1952) 562.

79. I. M. Dawson and D. H. Watson, Proc. Roy. Soc., 239A (1957) 349.

80. A. R. Lang, J. App. Phys., 28 (1957) 497.

81. C. E. Birchenall and H. M. Schadel, Jr., TAIME, 188 (1950) 1134.

82. J. J. Gilman and W. G. Johnston, J. App. Phys., 27 (1956) 1018. 83. W. T. Read, "Dislocations in Crystals," McGraw-Hill Book Co..,
New York (1953). 84. H. A. Jones, I. Langmuir and G. M. J. Mackay, Phys. Rev•, 30
(1927) 201.

85. R. S. Bradley and P. Volans, Proc. Roy. Soc., 217A (1953) 508.

86. F. G. Allen, Bell Laboratories, private communication.

87. G. Wesse1, Z. Physik, 130 (1951) 539.

88. G. W. Sears, J. Chem. Phys., 23 (1955) 1630.

89. G. W. Sears, Acta Met., 3 (1955) 367. 
90. H. Howard, private communication.

91. H. Lambot, L. Vassamillet, and J. Dejace, Acta Met., 3 (1955) 150.

92. H. Lambot, L. Vassamillet, and J. Dejace, ibid, I (1953) 711.

93. F. W. Sears, "Thermodyamics," Addisonmesley Publishing Co., Cambridge, Mass. (1953). 


\section{APPENDIX I}

\section{Computer Solution of Ledge Spacing}

Since equation (43) has no explicit solution, the equation was set up on a computor using the following assumptions:

$$
\begin{aligned}
& \text { a. Initially } \lambda_{10}=0, \lambda_{10}+\delta \lambda_{+}=\lambda_{20}=\infty \text { (see Fig. 3) } \\
& \text { b. Incremental increases of time } \tau=0.001 \text { second are assumed. }
\end{aligned}
$$
Thus at $t=1, \nabla_{11}=1 / 2 \nabla_{\max }$ from equation (39) At $t=2$, equation (39) becomes:

$$
\nabla_{12}=1 / 2 \nabla_{\max }\left[1+\tanh \left(\frac{Q \lambda_{11}}{2}\right)\right]
$$

But

$$
\lambda_{11}=\nabla_{11} \tau
$$

so that

$$
\nabla_{\text {In }}=1 / 2 \nabla_{\operatorname{mox}}\left[1+\tanh \left(\frac{Q \lambda_{1 n}-1}{2}\right)\right]
$$

and

$$
\lambda_{\text {ln }}=v_{\text {ln }} \tau \text {. }
$$

The results of the above iteration performed on the computor are shown in Figures Al and A2. It can be seen that the confirmation of the limiting expression, equation (28), is good, i.e. $\lambda \rightarrow \lambda_{0}$ and a $\lambda / \mathrm{d} x \rightarrow 0$ at $x \cong 10 \boldsymbol{\lambda}_{0}$.

It would be expected that agreement would be better if equation (43), involving the second order term $\mathrm{d}^{2} \lambda / \mathrm{dx}^{2}$ could be evaluated by inserting into equation (43) the approximations $\lambda=\boldsymbol{\lambda}_{n}$, $\mathrm{d} \lambda / \mathrm{dx}=\frac{\lambda_{n+1}=\lambda_{n}}{\lambda_{n}}$, and $\frac{\mathrm{d}^{2} \lambda}{\mathrm{d} \mathrm{x}^{2}}=\frac{\lambda_{n+1}-\lambda_{n}-1-2 \lambda_{n}}{\lambda_{n}{ }^{2}}$,

* The first subscript refers to the ledge in question, the second to the time increment. 


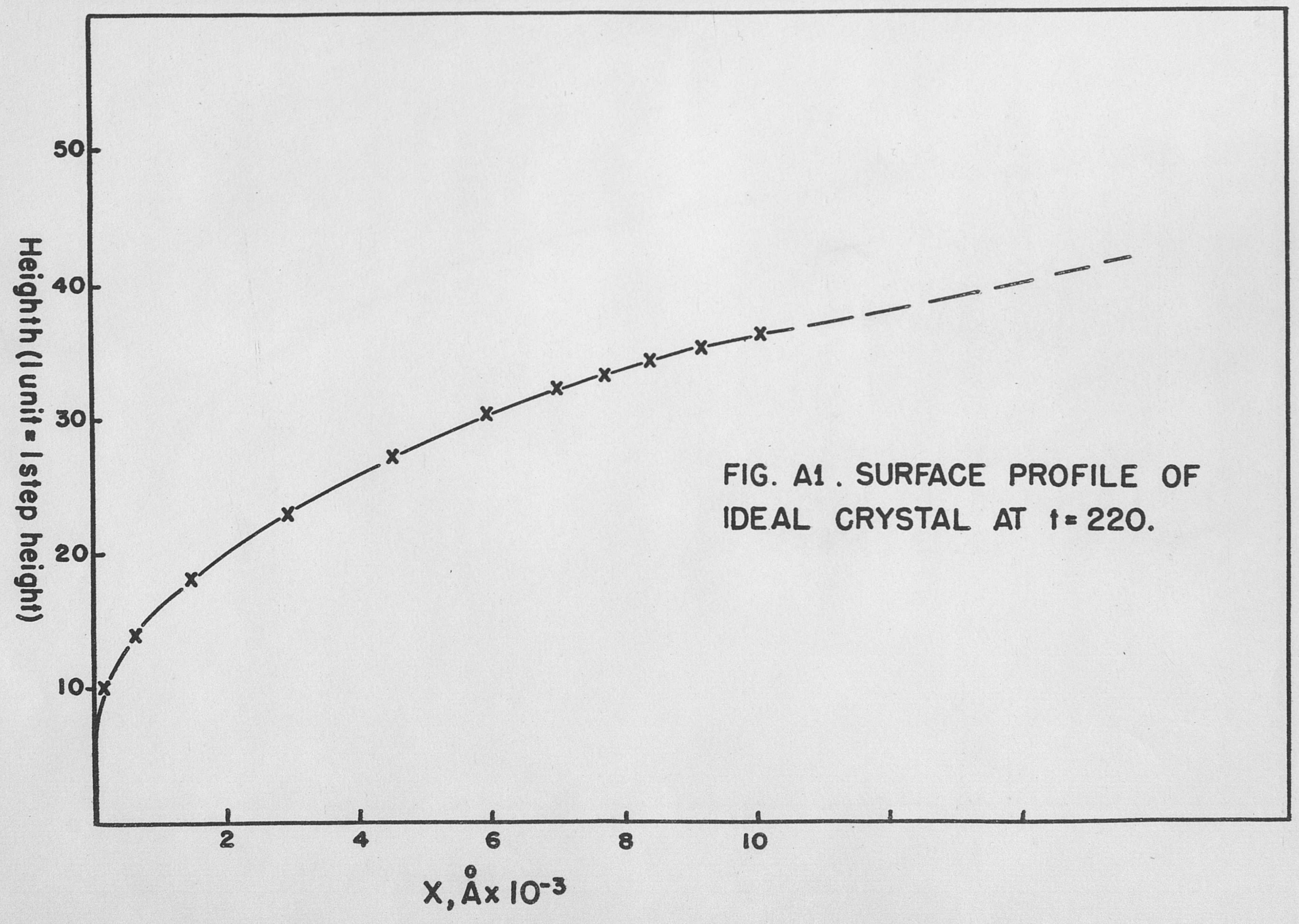




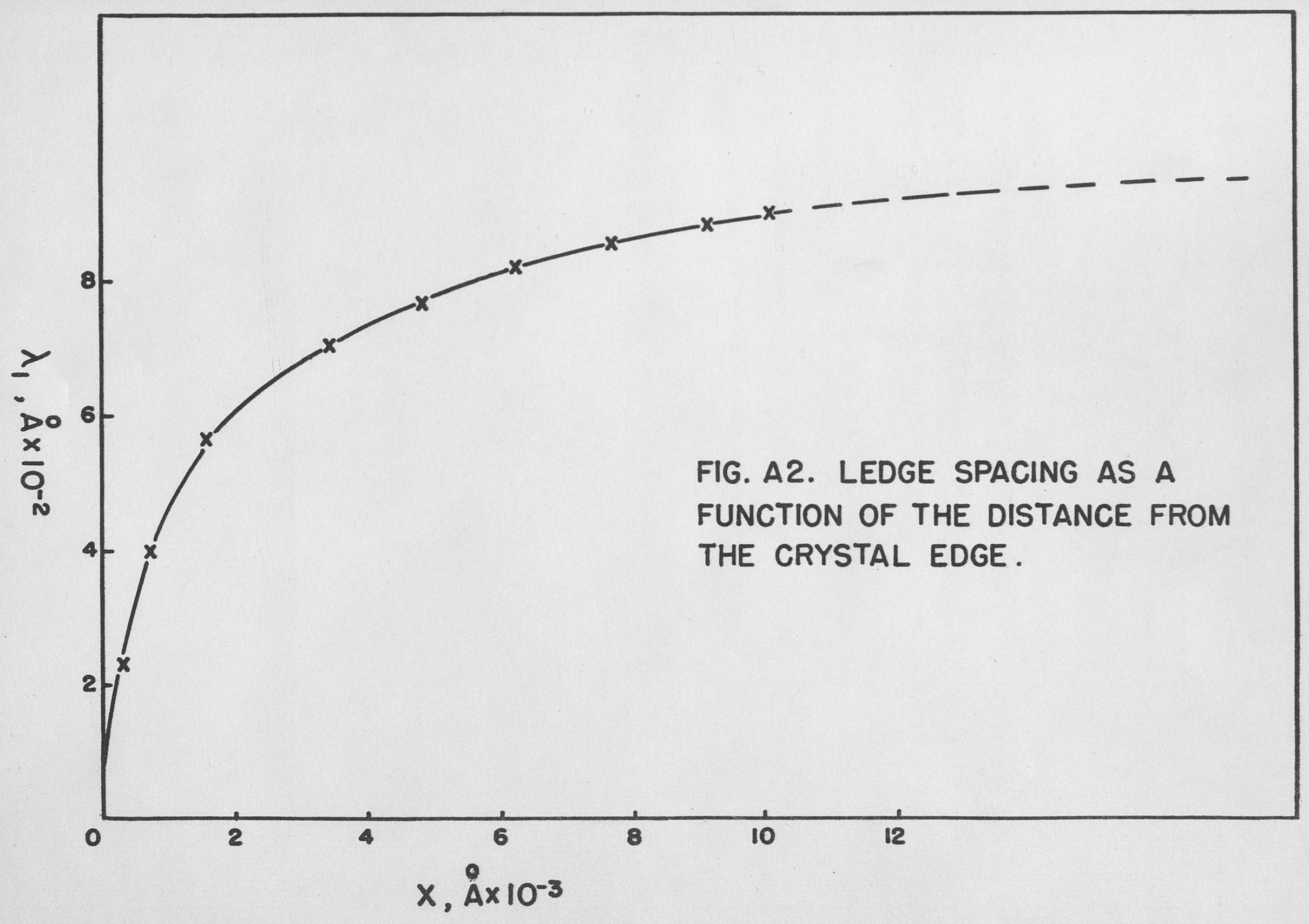


yielding

$\frac{\lambda_{n+1}-\lambda_{n}}{\lambda_{n}}=\frac{\tanh \frac{Q}{4}\left(3 \lambda_{n+1}+\lambda_{n-1}-2 \lambda_{n}\right)-\tanh \frac{Q}{4}\left(-\lambda_{n+1}+\lambda_{n-1}+2 \lambda_{n}\right)}{\tanh \frac{Q \lambda_{n}}{2}+\tanh \frac{Q}{4}\left(3 \lambda_{n+1}+\lambda_{n-1}-2 \lambda_{n}\right)}$

(A. 5)

which could be solved iteratively given $Q_{0} \lambda_{n}$, and $\lambda_{n=1}$. However, it was found that to get meaningful results, so many terms of the expansion would have had to be retained that the time of computation would have been prohibitive $(90)$. Nevertheless, it is apparent that the limits of equation (A 5) are of the required form.

An original copy of the computor results is on file in the office of Dr. G. M. Pound of the Metals Research Laboratory. 


\section{APPENDIX II}

\section{Single Crystal Orientation and Perfection}

The orientation of the single crystals as supplied was $\pm 1^{\circ}$. This was checked for the (110) crystal in a Laue back reflection camera and was found to be correct as shown in Figure BI.

Lattice perfection was determined by the method developed by L. Vassamillet et a. ${ }^{(91,92)}$ using a convergent $\mathrm{X}$-ray beam from a bent monochronometer. The estimate of dislocations for (100), (111), and (110) single crystal surfaces was $\cong 10^{8}$ per $\mathrm{cm}^{2}$ in each case. Figure B2 shows the (200) reflection of the (100) crystal at $50 \mathrm{~cm}$. from the specimen. This estimate is subject to the assumption that the distribution of dislocations in a face centered cubic metal is isotropic.

The assistance of Messrs. I. Vassamillet and W. H. Robinson of the Physics Department, Carnegie Institute of Technology, in carrying out this portion of the work is acknowledged. 


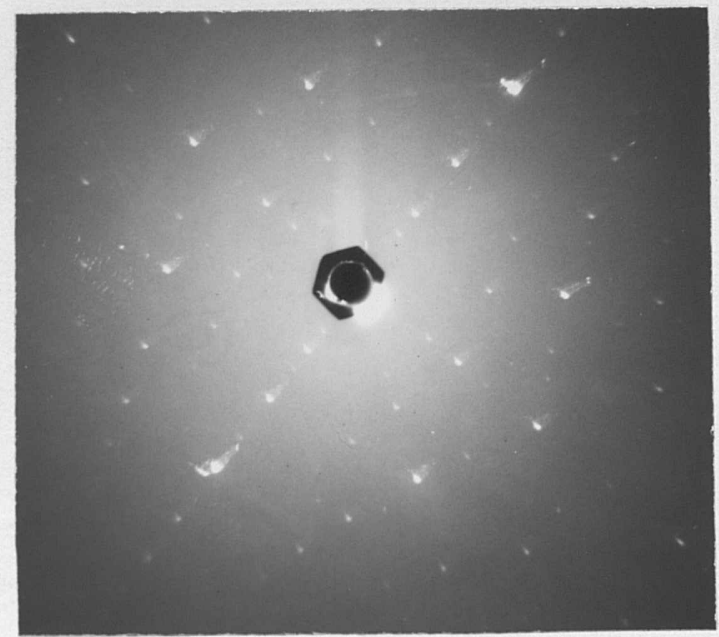

Figure B1. Laue back reflection from the $\{110\}$ pole of $\{110\}$ cylinder single crystal, target to film distance of $3 \mathrm{~cm}$.

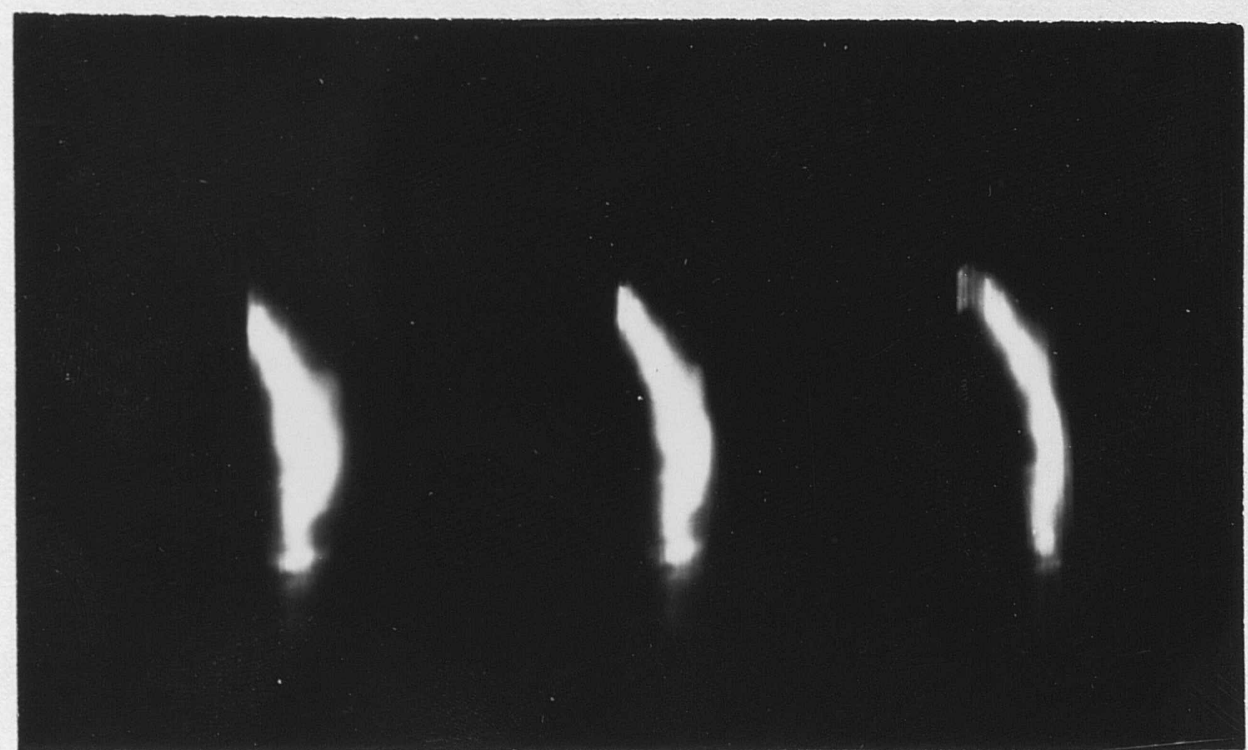

Figure B2. $\{200\}$ reflection from $\{100\}$ spherical single crystal, convergent beam, target to film distance of $50 \mathrm{~cm}$. 


\section{APPENDIX III}

Derivation of the Ideal Equation for Evaporation of a Sphere

The surface to volume ratio of a sphere is

$$
A / V=\frac{4 \pi r^{2}}{4 / 3 \pi r^{3}}=3 / r
$$

where $r$ is the radius of the sphere. The evaporation rate is given by

$$
J=-\frac{d m}{d t}=-\bar{\rho} \frac{d v}{d t}=-4 \pi r^{2} \bar{\rho} \frac{d r}{d t}
$$

where $\mathrm{m}$ is the mass of the sphere, $\bar{\rho}$ the density, and $\mathrm{v}$ the volume. From the kinetic theory of gases,

$$
J=\frac{\mathrm{pM}}{\sqrt{2 \pi \mathrm{MRT}}} \cdot 4 \pi r^{2}
$$

where $p$ is the vapor pressure of the substance in question, M the molem cular weight, and $R$ the gas constant. Equating (C 2) and (c 3) and integrating one obtains

$$
r=-\frac{1}{\bar{\rho}} \frac{p \mathbb{M}}{\sqrt{2 \pi M R T}} t+r_{0}
$$

where $r_{0}$ is the original radius of the sphere. From (c 2) and (c 3 )

$$
m=-\frac{4 \pi \mathrm{pM}}{\sqrt{2 \pi \mathrm{MRT}}} \int_{0}^{t} r^{2} d t .
$$

Substituting ( $C$ 4) in (c 5) and integrating, the final expression is obtained: 
$-101=$

$$
\begin{aligned}
m= & -\frac{4 \pi}{3 \bar{\rho}^{2}}\left(\frac{p M}{\sqrt{2 \pi M R T}}\right)^{3} t^{3}+\frac{4 r_{0} \pi}{\vec{\rho}}\left(\frac{p M}{\sqrt{2 \pi M R T}}\right)^{2} t^{2} \\
& -4 \pi r_{0}^{2}\left(\frac{p M}{\sqrt{2 \pi M R T}}\right) t+\frac{4 \pi r_{0}^{3} \bar{\rho}}{3} .
\end{aligned}
$$




\section{APPENDIX IV}

\section{Correction for Mean Free Path of Silver Vapor}

(93)

From statistical mechanics - the approximate expression for the mean free path of silver in vacuum is

$$
\bar{\lambda}=\frac{0.707}{\sigma_{n}}=\frac{0.707 \mathrm{kT}}{\pi\left(r_{1}+r_{2}\right)^{2} \times 10^{-16}}, \quad \text {, (D I) }
$$

where $n$ is the density of the gas, $r_{1}$ and $r_{2}$ are the radii of the silver atom and nitrogen molecule respectively, $p$ is the pressure of air at absolute temperature $\mathrm{T}$, and $\mathrm{k}$ is Boltzmann's constant. Taking the temperature of the gas in the system as the temperature of the container walls, $125^{\circ} \mathrm{C}$, and taking $r_{1}=1.8 \times 10^{-8} \mathrm{~cm}$ and $r_{2}=1.44 \mathrm{x}$ $10^{-8} \mathrm{~cm},(94)$ c.f. $r=1.40$ for oxygen, one obtains

$$
\bar{\lambda}=0.00882 \mathrm{p}^{-1} \text {. }
$$

for $\mathrm{p}$ in dynes $/ \mathrm{cm}^{2}$. The survival equation for the number of molecules $N$ arriving at a target removed a distance $b$ from the source where $N_{0}$ atoms depart is $(93)$

$$
N / N_{0}=\exp -(b / \bar{\lambda})
$$

Results are shown in Table D I for $N / N_{0}$ as a function of $p$ taking $b$ as $5 \mathrm{~cm}$.

Table D I

Mean Free Path of Silver in Vacuum

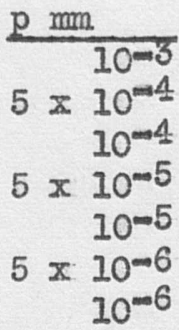

\begin{tabular}{c}
$\overline{\bar{\lambda} \mathrm{cm}}$ \\
\hline 8.82 \\
17.64 \\
88.2 \\
176.4 \\
882 \\
1764 \\
8820
\end{tabular}

\begin{tabular}{l}
$N / N_{0} \%$ \\
\hline 56.8 \\
75.4 \\
94.5 \\
97.2 \\
99.4 \\
99.7 \\
99.26
\end{tabular}

